\title{
The Modified Fourier-Ritz Approach for the Free Vibration of Functionally Graded Cylindrical, Conical, Spherical Panels and Shells of Revolution with General Boundary Condition
}

\author{
Lijie Li, ${ }^{1}$ Haichao Li, ${ }^{2}$ Fuzhen Pang, ${ }^{2,3}$ Xueren Wang, ${ }^{3}$ Yuan Du, ${ }^{2}$ and Shuo $\mathrm{Li}^{2}$ \\ ${ }^{1}$ College of Computer Science and Technology, Harbin Engineering University, Harbin 150001, China \\ ${ }^{2}$ College of Shipbuilding Engineering, Harbin Engineering University, Harbin 150001, China \\ ${ }^{3}$ Naval Academy of Armament, Beijing 100161, China \\ Correspondence should be addressed to Haichao Li; lihaichao@hrbeu.edu.cn
}

Received 30 June 2017; Revised 2 September 2017; Accepted 17 September 2017; Published 31 October 2017

Academic Editor: Francesco Tornabene

Copyright (C) 2017 Lijie Li et al. This is an open access article distributed under the Creative Commons Attribution License, which permits unrestricted use, distribution, and reproduction in any medium, provided the original work is properly cited.

\begin{abstract}
The aim of this paper is to extend the modified Fourier-Ritz approach to evaluate the free vibration of four-parameter functionally graded moderately thick cylindrical, conical, spherical panels and shells of revolution with general boundary conditions. The first-order shear deformation theory is employed to formulate the theoretical model. In the modified Fourier-Ritz approach, the admissible functions of the structure elements are expanded into the improved Fourier series which consist of two-dimensional (2D) Fourier cosine series and auxiliary functions to eliminate all the relevant discontinuities of the displacements and their derivatives at the edges regardless of boundary conditions and then solve the natural frequencies by means of the Ritz method. As one merit of this paper, the functionally graded cylindrical, conical, spherical shells are, respectively, regarded as a special functionally graded cylindrical, conical, spherical panels, and the coupling spring technology is introduced to ensure the kinematic and physical compatibility at the common meridian. The excellent accuracy and reliability of the unified computational model are compared with the results found in the literatures.
\end{abstract}

\section{Introduction}

As the based structure elements, functionally graded cylindrical, conical, spherical panels and shells have a wide application in various engineering constructions, such as aircraft, space vehicles, and structures of military industries. Therefore, the knowledge of vibration characteristics of the functionally graded cylindrical, conical, spherical panels and shells is of particular importance for the predesign of the engineering structures.

So far, a huge amount of researches focused on the vibration analysis of functionally graded cylindrical, conical, spherical panels and shells by using different methods and shell theories. Tornabene and Viola [1-3] studied the free vibration of the moderately thick four-parameter functionally graded conical, cylindrical shells and annular plates under some classical boundary conditions by means of the generalized differential quadrature method on the basis of the first-order shear deformation theory. Zhao and Liew $[4,5]$ presented the element-free kp-Ritz method to investigate the free vibration analysis of mechanical and thermal buckling of functionally graded conical shell panels with classical boundary conditions. Aragh and Hedayati [6] dealt with the free vibration and static response of a twodimensional functionally graded (2D FGM) metal/ceramic open cylindrical shell with classical boundary conditions by using 2D generalized differential quadrature method. Su et al. [7-9] applied the modified Fourier series and Rayleigh-Ritz method to analyze the free vibrations of functionally graded open and closed shells including cylindrical, conical, and spherical ones with general boundary conditions based on first-order shear deformation theory. Sofiyev and Kuruoglu [10-13] presented a theoretical approach on the basis of the Galerkin method to solve vibration problems of functionally graded (FG) truncated and complete conical shells under mixed classical boundary conditions and resting on elastic 
foundations. Kim [14] used an analytical method to investigate the free vibration characteristics of FGM cylindrical shells partially resting on elastic foundation with an oblique edge. Jin et al. $[15,16]$ presented a Haar Wavelet Discretization (HWD) method-based solution approach for the free vibration analysis of functionally graded (FG) cylindrical, spherical, and parabolic shells of revolution with arbitrary boundary conditions. Hosseini-Hashemi et al. [17] used the state space approach to investigate the free vibration of Levy-type thick functionally graded (FG) circular cylindrical shell panels to identify the validity range of two common shell theories namely Donnell and Sanders theories. Iqbal et al. [18] applied the wave propagation approach to study the vibration characteristics of functionally graded material circular cylindrical shells with classical boundary conditions. Shah et al. [19] employed the wave propagation method to study the vibrations of functionally graded cylindrical shells based on the Winkler and Pasternak foundations. Loy et al. $[20,21]$ studied the vibration of cylindrical shells made of a functionally gradient material (FGM) composed of stainless steel and nickel by means of the Rayleigh-Ritz method under some selected boundary conditions. Najafizadeh and Isvandzibaei [22] used the Rayleigh-Ritz method and third order shear deformation shell theory to analyze the free vibrations of the thin cylindrical shells under ring supports and classical boundary conditions. Naeem et al. [23] applied the Ritz formulation to study the vibration frequencies of functionally graded circular cylindrical shells with classical boundary conditions. Taghvaeipour et al. [24] presented a new finite element formulation to study the structural analysis of functionally graded hollow cylinders. Pradyumna and Bandyopadhyay [25] employed the finite element method to investigate the free vibration analysis of functionally graded curved panels including the cylindrical and spherical panels based on the higher-order shear deformation theory. Neves et al. [26] dealt with free vibration problems of functionally graded shells with classical boundary conditions based on the higher-order shear deformation theory and the radial basis functions collocation. Qu et al. [27] described a general formulation for free, steady-state, and transient vibration analyses of functionally graded shells of revolution subjected to arbitrary boundary conditions by means of a modified variational principle in conjunction with a multisegment partitioning procedure. Santos et al. [28, 29] developed a semianalytical axisymmetric finite element model using the 3D linear elastic theory to study the free vibrations of functionally graded cylindrical shells made up of isotropic properties. Vel [30] presented an exact elasticity solution for the free and forced vibration of functionally graded cylindrical shells with classical boundary conditions.

The above review indicates that there exist some literatures on the free vibration of a functionally graded cylindrical, conical, spherical panels and shells, and those numerical results are very useful for the practical project application. However, from the literatures review, we also know that the most existing numerical solutions for the title problem usually take account of a onefold computational model instead of unified computational model. For instance, Su et al. [7-9] divided the open and closed functionally graded cylindrical, conical, spherical shells to study and resort different admissible functions by means of the modified Fourier series technology. However, in practical project application, the structural forms are generally unknown. Thus, the establishment of a unified, efficient, and accurate computational model for free vibration analysis of functionally graded cylindrical, conical, spherical panels and shells with general boundary conditions is necessary and significant.

In previous study, an improved Fourier series method was proposed by $\mathrm{Li}[31,32]$ to determine the vibration of a single beam under arbitrary boundary conditions. The method has been subsequently exploited to determine the vibration behaviors of structures [33-48]. Recently, a new method of Fourier-Ritz approach was proposed by Jin et al. [49], based on the improved Fourier series method to analyze the free vibration of laminated functionally graded shallow shells with general boundary conditions. In contrast to most existing techniques, the modified Fourier-Ritz method can be universally applicable to a variety of boundary conditions including all the classical cases, elastic restraints, and their combinations without the need of making any changes of the solution procedure. The purpose of this paper is to extend a modified Fourier-Ritz approach to presents a unified computational model for the free vibration of fourparameter functionally graded moderately thick cylindrical, conical, spherical panels and shells of revolution with general boundary conditions on the basis of the first-order shear deformation theory. Applying the modified Fourier-Ritz approach, the admissible functions of the structure elements are expanded into the improved Fourier series which consist of two-dimensional (2D) Fourier cosine series and auxiliary functions to eliminate all the relevant discontinuities of the displacements and their derivatives at the edges regardless of boundary conditions and then solving the natural frequencies by means of the Ritz method. In order to obtain the unified computational model of the functionally graded cylindrical, conical, spherical panels and shells of revolution, as one merit of this paper, the coupling spring technology is introduced to ensure the kinematic and physical compatibility at the common meridian, if a complete shell of revolution needs considering. The excellent accuracy and reliability of the unified computational model are compared with the results found in the literatures.

\section{Theoretical Formulations}

2.1. Description of the Model. As shown in Figure 1, the basic configuration of the problem considered here is an FG shell. An orthogonal curvilinear coordinate system composed of coordinates $\alpha, \beta$, and $z$ is located on the reference surface ( $z=0$ ) of the shell. $R_{\alpha}$ and $R_{\beta}$ denote the values of the principal radii of curvature of the reference surface along $\alpha$ and $\beta$ axes, respectively. $L_{\alpha}$ and $L_{\beta}$ denote the lengths in $\alpha$ and $\beta$ directions, respectively. The thickness of shell element is represented by $h$. As shown in Figure 2, the geometry of panels and shells considered in this paper is a surface of revolution with a cylindrical, conical, and spherical meridian. Based on Figure 2, the coordinate systems and quantities $R_{\alpha}$, $R_{\beta}, L_{\alpha}$, and $L_{\beta}$ are given as follows: for cylindrical meridian, 

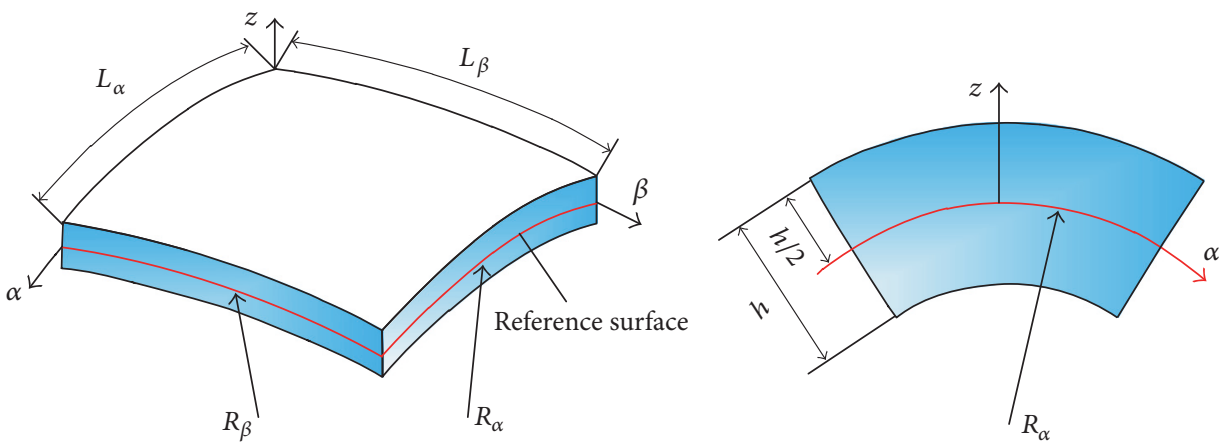

FIGURE 1: Geometry and notations of a FGM shell.

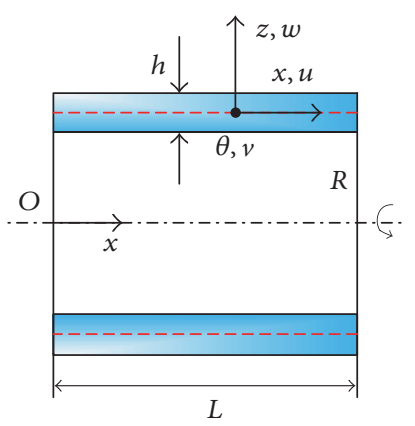

(a)

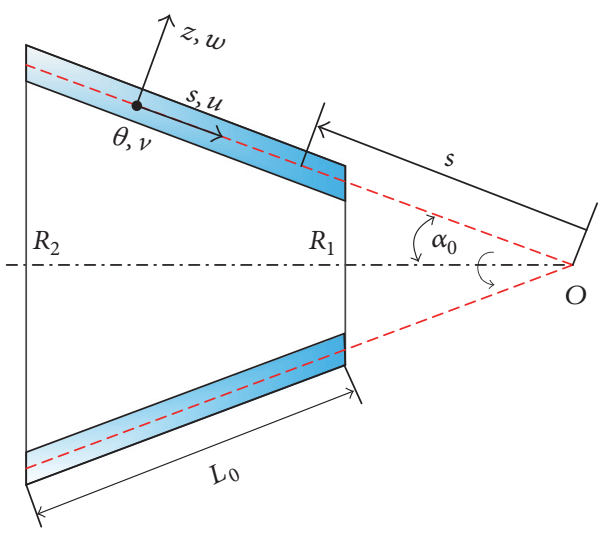

(b)

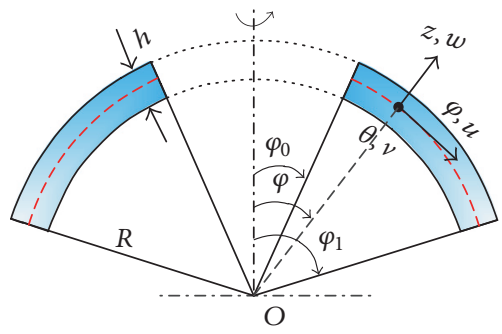

(c)

FIGURE 2: Definition of shell coordinate system: (a) cylindrical shell; (b) conical shell; (c) spherical shell.

$\alpha=x, \beta=\theta, R_{\alpha}=\infty, R_{\beta}=R, L_{\alpha}=L$, and $L_{\beta}=\phi(0 \leq$ $\phi \leq 2 \pi)$; for conical meridian, $\alpha=s, \beta=\theta, R_{\alpha}=\infty$, $R_{\beta}=s \times \tan \alpha_{0}, L_{\alpha}=L_{0}$, and $L_{\beta}=\phi(0 \leq \phi \leq 2 \pi)$; for spherical meridian, $\alpha=\varphi, \beta=\theta, R_{\alpha}=R_{\beta}=R$, $L_{\alpha}=\varphi\left(\varphi=\varphi_{1}-\varphi_{0}\right)$, and $L_{\beta}=\phi(0 \leq \phi \leq 2 \pi)$. The details of the geometric configuration of the FG panels and shells of revolution structures for verifying the accuracy and versatility of the proposed approach are shown in Figure 3.

2.2. Kinematic Relations and Stress Resultants. According to the FSDT assumptions, the displacement components of an arbitrary point in the FGM panels and shells are expressed in terms of the displacements and rotations of the reference surface, as given as follows:

$$
\begin{aligned}
U(\alpha, \beta, z, t) & =u_{0}(\alpha, \beta, t)+z \psi_{\alpha}(\alpha, \beta, t) \\
V(\alpha, \beta, z, t) & =v_{0}(\alpha, \beta, t)+z \psi_{\beta}(\alpha, \beta, t) \\
W(\alpha, \beta, z, t) & =w_{0}(\alpha, \beta, t),
\end{aligned}
$$

where $u_{0}, v_{0}$, and $w$ denote the displacements of corresponding point on reference surface in the $\alpha, \beta$, and $z$ directions, respectively. $\psi_{\alpha}$ and $\psi_{\beta}$ are the rotations of the normal to the reference surface about the $\beta$ and $\alpha$ direction, respectively, and $t$ is the time. Relationships between strains and displacements along the shell reference (middle) surface $(z=0)$ are represented by the following:

$$
\begin{aligned}
\varepsilon_{\alpha}^{0} & =\frac{1}{A} \frac{\partial u_{0}}{\partial \alpha}+\frac{v_{0}}{A B} \frac{\partial A}{\partial \beta}+\frac{w_{0}}{R_{\alpha}}, \\
\varepsilon_{\beta}^{0} & =\frac{1}{B} \frac{\partial v_{0}}{\partial \beta}+\frac{u_{0}}{A B} \frac{\partial B}{\partial \alpha}+\frac{w_{0}}{R_{\beta}}, \\
\gamma_{\alpha \beta}^{0} & =\frac{B}{A} \frac{\partial}{\partial \alpha}\left(\frac{v_{0}}{B}\right)+\frac{A}{B} \frac{\partial}{\partial \beta}\left(\frac{u_{0}}{A}\right) \\
\kappa_{\alpha} & =\frac{1}{A} \frac{\partial \psi_{\alpha}}{\partial \alpha}+\frac{\psi_{\beta}}{A B} \frac{\partial A}{\partial \beta}, \\
\kappa_{\beta} & =\frac{1}{B} \frac{\partial \psi_{\beta}}{\partial \beta}+\frac{\psi_{\alpha}}{A B} \frac{\partial B}{\partial \alpha}, \\
\kappa_{\alpha \beta} & =\frac{B}{A} \frac{\partial}{\partial \alpha}\left(\frac{\psi_{\beta}}{B}\right)+\frac{A}{B} \frac{\partial}{\partial \beta}\left(\frac{\psi_{\alpha}}{A}\right) \\
\gamma_{\alpha z}^{0} & =\psi_{\alpha}-\frac{u_{0}}{R_{\alpha}}+\frac{1}{A} \frac{\partial w_{0}}{\partial \alpha}, \\
\gamma_{\beta z}^{0} & =\psi_{\beta}-\frac{v_{0}}{R_{\beta}}+\frac{1}{B} \frac{\partial w_{0}}{\partial \beta},
\end{aligned}
$$




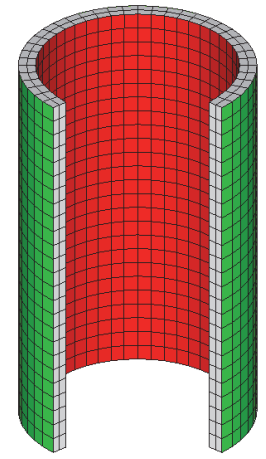

Cylindrical panels

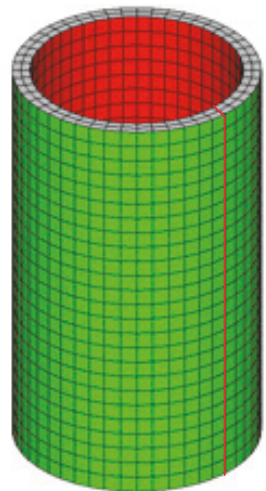

Cylindrical shells

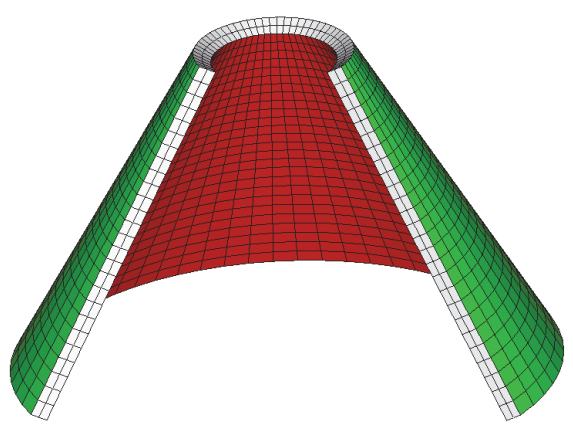

Conical panels

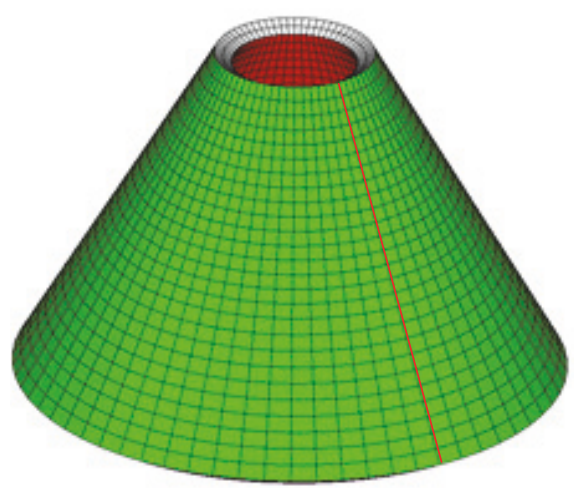

Conical shells

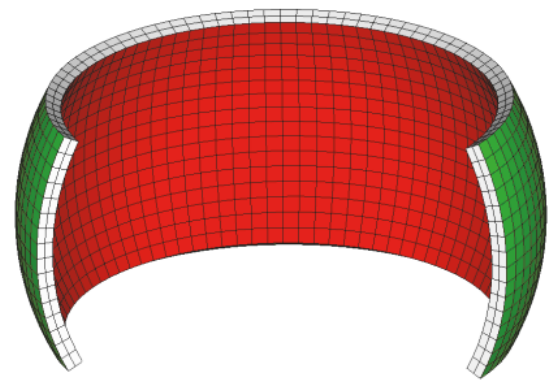

Spherical panels

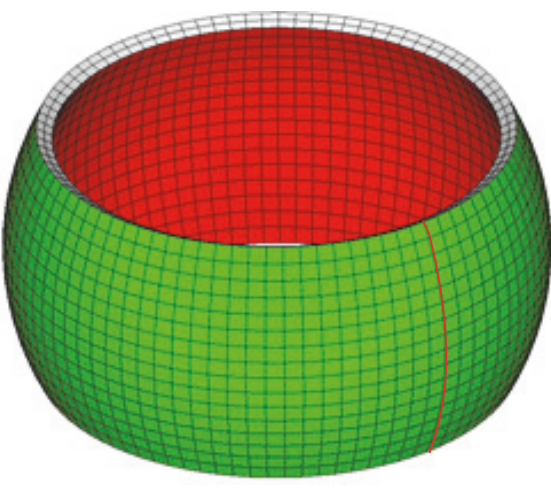

Spherical shells

FIGURE 3: FG panel and shell structures.

where the symbol $A$ and $B$ are the Lamé parameters. According to Figure 2, the Lamé parameters of the above types panels and shells can be defined as follows: for case of the cylindrical meridian, $A=1$ and $B=R$; for case of the conical meridian, $A=1$ and $B=s \times \sin \alpha_{0}$; for case of the spherical meridian, $A=R$ and $B=R \times \sin \varphi$.

The constitutive equations related the force and moment resultants to strains and curvatures of the reference surface are given in the matrix form:

$$
\begin{aligned}
& {\left[\begin{array}{c}
N_{\alpha} \\
N_{\beta} \\
N_{\alpha \beta} \\
M_{\alpha} \\
M_{\beta} \\
M_{\alpha \beta}
\end{array}\right]=\left[\begin{array}{cccccc}
A_{11} & A_{12} & 0 & B_{11} & B_{12} & 0 \\
A_{12} & A_{11} & 0 & B_{12} & B_{11} & 0 \\
0 & 0 & A_{66} & 0 & 0 & B_{66} \\
B_{11} & B_{12} & 0 & D_{11} & D_{12} & 0 \\
B_{12} & B_{11} & 0 & D_{12} & D_{11} & 0 \\
0 & 0 & B_{66} & 0 & 0 & D_{66}
\end{array}\right]\left[\begin{array}{c}
\varepsilon_{\alpha}^{0} \\
\varepsilon_{\beta}^{0} \\
\gamma_{\alpha \beta}^{0} \\
\kappa_{\alpha} \\
\kappa_{\beta} \\
\kappa_{\alpha \beta}
\end{array}\right]} \\
& {\left[\begin{array}{c}
Q_{\alpha} \\
Q_{\beta}
\end{array}\right]=\left[\begin{array}{cc}
\kappa A_{66} & 0 \\
0 & \kappa A_{66}
\end{array}\right]\left[\begin{array}{c}
\gamma_{\alpha z}^{0} \\
\gamma_{\beta z}^{0}
\end{array}\right],}
\end{aligned}
$$

where $N_{\alpha}, N_{\beta}$, and $N_{\alpha \beta}$ are the in-plane force resultants, $M_{\alpha}$, $M_{\beta}$, and $M_{\alpha \beta}$ are moment resultants, and $Q_{\alpha}$ and $Q_{\beta}$ are transverse shear force resultants. The shear correction factor $\kappa$ is computed such that the strain energy due to transverse shear stresses in (3) equals the strain energy due to the true transverse stresses predicted by the three-dimensional elasticity theory. In application, the shear correction factor does not have to be the same in different geometric directions and depended on many factors such as the geometric and material parameters [50]. However, the aim of in this paper is to study the vibration analysis of vibration analysis of functionally graded cylindrical, conical, spherical panels and shells with general boundary conditions. Thus, in order simplify this study and based on existing literature $[40,51]$, the shear correction factor $\kappa$ selected a generic parameter $\kappa=5 / 6$ in the next calculation. $A_{i j}, B_{i j}$, and $D_{i j}(i, j=1,2$ and 6 ) are the extensional, extensional-bending coupling and bending stiffness, and they are, respectively, expressed as

$$
\left(A_{i j}, B_{i j}, D_{i j}\right)=\int_{-h / 2}^{h / 2} Q_{i j}(z)\left(1, z, z^{2}\right) d z
$$

where the elastic constants $Q_{i j}(z)$ are functions of thickness coordinate $z$, which are defined as

$$
\begin{aligned}
Q_{11}(z) & =\frac{E(z)}{1-\mu^{2}(z)}, \\
Q_{12}(z) & =\frac{\mu(z) E(z)}{1-\mu^{2}(z)}, \\
Q_{66}(z) & =\frac{E(z)}{2(1+\mu(z))} .
\end{aligned}
$$


Typically, the functionally graded materials are made of two or more constituent phases which have smooth and continuous mechanical behavior in one or more directions. In this paper, it is assumed that the FG panels and shells are made of a mixture consisting of ceramic and metal. Young's modulus $E(z)$, density $\rho(z)$, and Poisson's ratio $\mu(z)$ are assumed to vary continuously through the panels thickness and can be expressed as a linear combination:

$$
\begin{aligned}
& E(z)=\left(E_{c}-E_{m}\right) V_{c}+E_{m} \\
& \rho(z)=\left(\rho_{c}-\rho_{m}\right) V_{c}+\rho_{m} \\
& \mu(z)=\left(\mu_{c}-\mu_{m}\right) V_{c}+\mu_{m}
\end{aligned}
$$

in which the subscripts $c$ and $m$ represent the ceramic and metallic constituents, respectively. And the volume fraction $V_{c}$ follows the two general four-parameter power-law distributions [1]:

$$
\begin{aligned}
\operatorname{FGM}_{\mathrm{I}(a / b / c / p)}: V_{c} & =\left[1-a\left(\frac{1}{2}+\frac{z}{h}\right)+b\left(\frac{1}{2}+\frac{z}{h}\right)^{c}\right]^{p} \\
\mathrm{FGM}_{\mathrm{II}(a / b / c / p)}: V_{c} & =\left[1-a\left(\frac{1}{2}-\frac{z}{h}\right)+b\left(\frac{1}{2}-\frac{z}{h}\right)^{c}\right]^{p},
\end{aligned}
$$

where $p$ is the power-law exponent and takes only positive values, $a, b$, and $c$ are the material parameters, and their values determine the volume fraction through the general fourparameter power-law distributions. In addition, for (7), the difference between the two general four-parameter powerlaw distributions is decided by $1 / 2-z / h$ and $1 / 2+z / h$. In order to get more intuitive description, Figure 4 shows the variations of the volume fraction $V_{c}$ for different distributions and values of the power-law exponent. Form Figure 4, we can know that the volume fraction $V_{c}$ with $\mathrm{FGM}_{\mathrm{I}}$ and $\mathrm{FGM}_{\mathrm{II}}$ is symmetric with reference to $z=0$. The volume fraction of all the constituent materials should add up to one; that is,

$$
V_{c}+V_{m}=1 \text {. }
$$

When the value of $p$ equals zero or infinity, the homogeneous isotropic material can be obtained as a special case of the functionally graded material.

The strain energy $\left(U_{s}\right)$ of the moderately thick functionally graded panels and shells of revolution can be defined as

$$
\begin{aligned}
U_{s} & =\frac{1}{2} \iiint_{V}\left\{N_{\alpha} \varepsilon_{\alpha}^{0}+N_{\beta} \varepsilon_{\beta}^{0}+N_{\alpha \beta} \gamma_{\alpha \beta}^{0}+M_{\alpha} \kappa_{\alpha}+M_{\beta} \kappa_{\beta}\right. \\
& \left.+M_{\varphi \beta} \kappa_{\varphi \beta}+Q_{\alpha} \gamma_{\alpha z}^{0}+Q_{\beta} \gamma_{\beta z}^{0}\right\} d V .
\end{aligned}
$$

Substituting (2) and (3) into (9), the strain energy expression of the structure can be written in terms of middle surface displacements and rotations. For convenience, the strain energy expression is divided into three components; that is, $U_{s}=U_{S}+U_{B}+U_{\mathrm{BS}}$, where $U_{S}, U_{\mathrm{BS}}$, and $U_{B}$ indicate Stretching, Bending, and Bending-Stretching coupling energy expressions, respectively.

$$
\begin{aligned}
& U_{S}=\frac{1}{2} \iint_{S}\left\{A_{11}\left(\frac{1}{A} \frac{\partial u_{0}}{\partial \alpha}+\frac{v_{0}}{A B} \frac{\partial A}{\partial \beta}+\frac{w_{0}}{R_{\alpha}}\right)^{2}\right. \\
& +A_{11}\left(\frac{1}{B} \frac{\partial v_{0}}{\partial \beta}+\frac{u_{0}}{A B} \frac{\partial B}{\partial \alpha}+\frac{w_{0}}{R_{\beta}}\right)^{2} \\
& +\kappa A_{66}\left(\psi_{\alpha}-\frac{u_{0}}{R_{\alpha}}+\frac{1}{A} \frac{\partial w_{0}}{\partial \alpha}\right)^{2} \\
& +A_{66}\left(\frac{B}{A} \frac{\partial}{\partial \alpha}\left(\frac{v_{0}}{B}\right)+\frac{A}{B} \frac{\partial}{\partial \beta}\left(\frac{u_{0}}{A}\right)\right)^{2} \\
& +2 A_{12}\left(\frac{1}{A} \frac{\partial u_{0}}{\partial \alpha}+\frac{v_{0}}{A B} \frac{\partial A}{\partial \beta}+\frac{w_{0}}{R_{\alpha}}\right) \\
& \cdot\left(\frac{1}{B} \frac{\partial v_{0}}{\partial \beta}+\frac{u_{0}}{A B} \frac{\partial B}{\partial \alpha}+\frac{w_{0}}{R_{\beta}}\right) \\
& \left.+\kappa A_{66}\left(\psi_{\beta}-\frac{v_{0}}{R_{\beta}}+\frac{1}{B} \frac{\partial w_{0}}{\partial \beta}\right)^{2}\right\} \\
& U_{\mathrm{BS}}=\frac{1}{2} \iint_{S}\left\{2 B_{11}\left(\frac{1}{A} \frac{\partial u_{0}}{\partial \alpha}+\frac{v_{0}}{A B} \frac{\partial A}{\partial \beta}+\frac{w_{0}}{R_{\alpha}}\right)\right. \\
& \cdot\left(\frac{1}{A} \frac{\partial \psi_{\alpha}}{\partial \alpha}+\frac{\psi_{\beta}}{A B} \frac{\partial A}{\partial \beta}\right) \\
& +2 B_{11}\left(\frac{1}{B} \frac{\partial v_{0}}{\partial \beta}+\frac{u_{0}}{A B} \frac{\partial B}{\partial \alpha}+\frac{w_{0}}{R_{\beta}}\right) \\
& \cdot\left(\frac{1}{B} \frac{\partial \psi_{\beta}}{\partial \beta}+\frac{\psi_{\alpha}}{A B} \frac{\partial B}{\partial \alpha}\right) \\
& +2 B_{12}\left(\frac{1}{A} \frac{\partial u_{0}}{\partial \alpha}+\frac{v_{0}}{A B} \frac{\partial A}{\partial \beta}+\frac{w_{0}}{R_{\alpha}}\right) \\
& \cdot\left(\frac{1}{B} \frac{\partial \psi_{\beta}}{\partial \beta}+\frac{\psi_{\alpha}}{A B} \frac{\partial B}{\partial \alpha}\right) \\
& +2 B_{12}\left(\frac{1}{B} \frac{\partial v_{0}}{\partial \beta}+\frac{u_{0}}{A B} \frac{\partial B}{\partial \alpha}+\frac{w_{0}}{R_{\beta}}\right) \\
& \cdot\left(\frac{1}{A} \frac{\partial \psi_{\alpha}}{\partial \alpha}+\frac{\psi_{\beta}}{A B} \frac{\partial A}{\partial \beta}\right) \\
& +2 B_{66}\left(\frac{B}{A} \frac{\partial}{\partial \alpha}\left(\frac{v_{0}}{B}\right)+\frac{A}{B} \frac{\partial}{\partial \beta}\left(\frac{u_{0}}{A}\right)\right) \\
& \left.\cdot\left(\frac{B}{A} \frac{\partial}{\partial \alpha}\left(\frac{\psi_{\beta}}{B}\right)+\frac{A}{B} \frac{\partial}{\partial \beta}\left(\frac{\psi_{\alpha}}{A}\right)\right)\right\} d S \\
& U_{B}=\frac{1}{2} \iint_{S}\left\{D_{11}\left(\frac{1}{A} \frac{\partial \psi_{\alpha}}{\partial \alpha}+\frac{\psi_{\beta}}{A B} \frac{\partial A}{\partial \beta}\right)^{2}\right. \\
& +D_{11}\left(\frac{1}{B} \frac{\partial \psi_{\beta}}{\partial \beta}+\frac{\psi_{\alpha}}{A B} \frac{\partial B}{\partial \alpha}\right)^{2}
\end{aligned}
$$




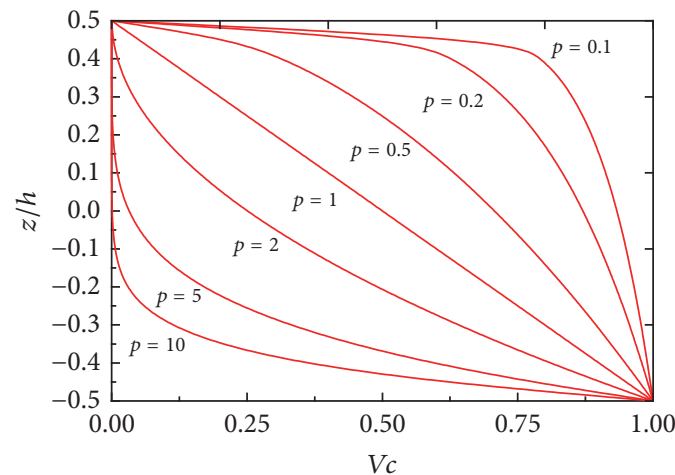

(a)

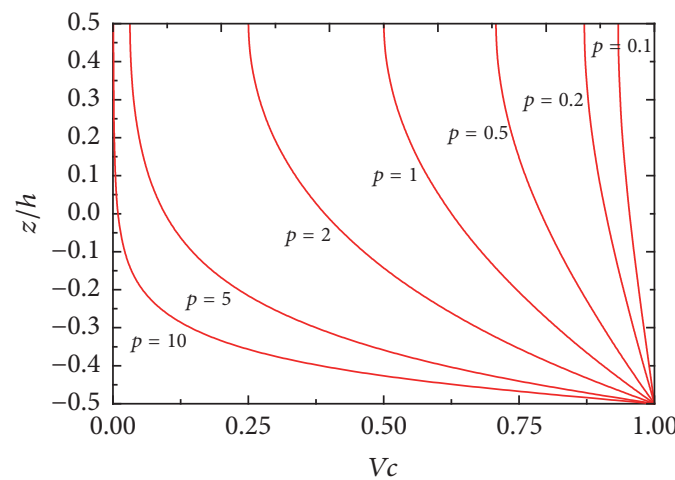

(c)

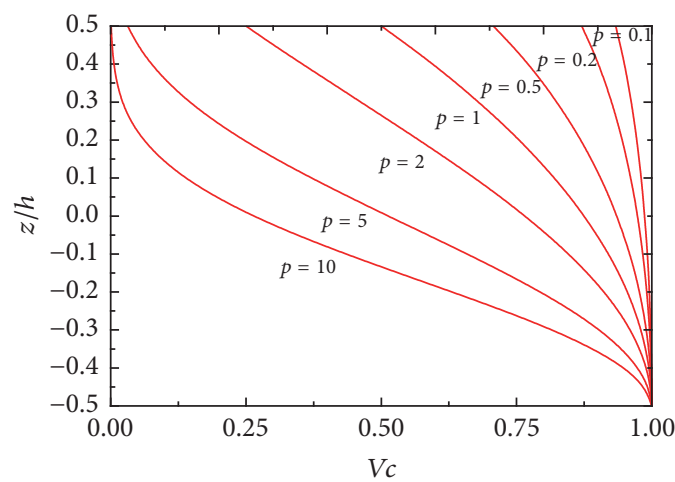

(e)

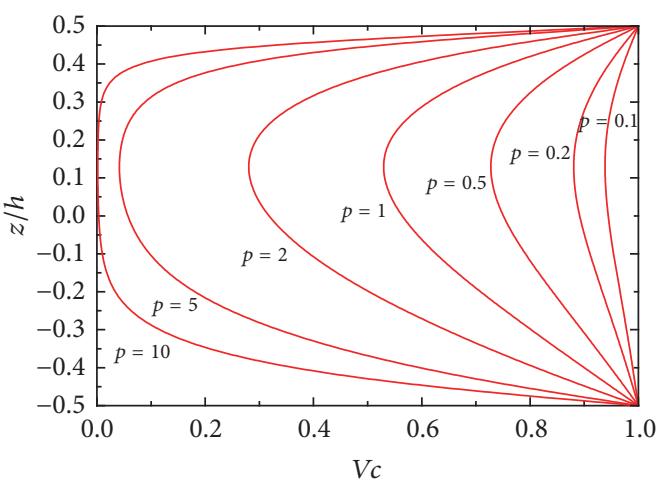

(g)

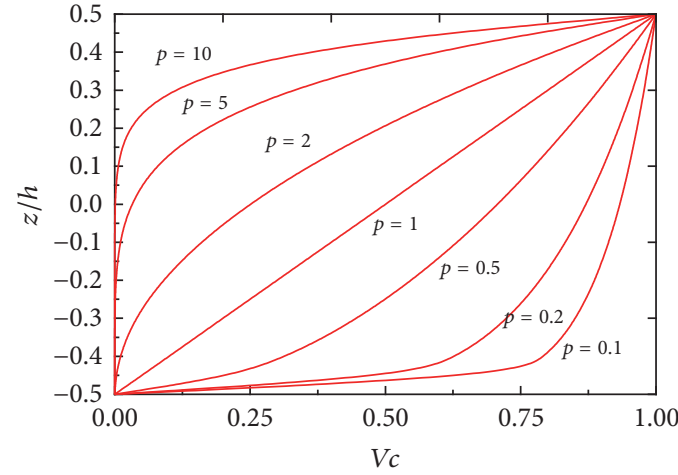

(b)

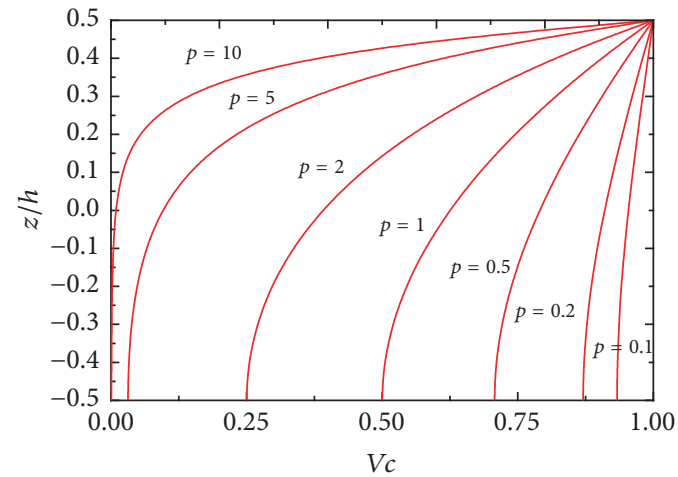

(d)

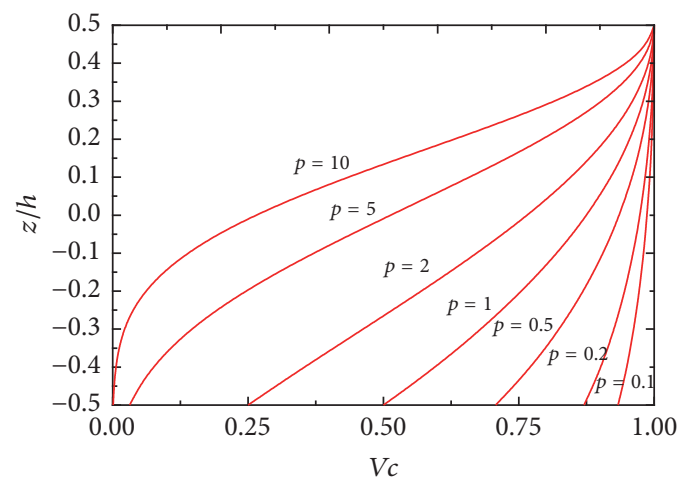

(f)

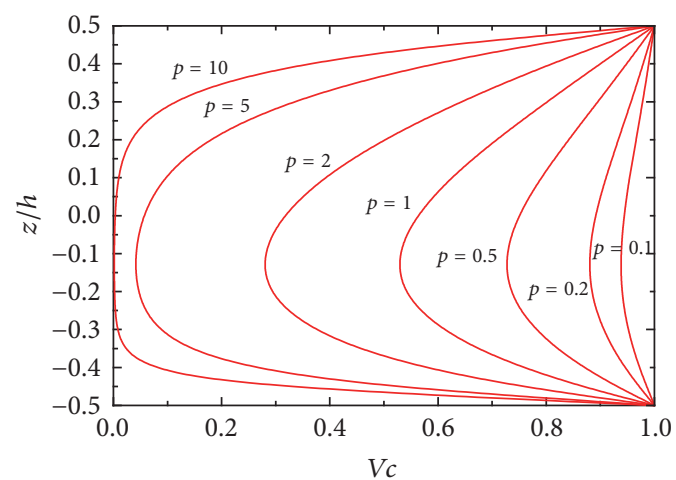

(h)

Figure 4: Variations of the volume fraction $V c$ through the shell thickness for different values of power-law exponent $p$ : (a) $\operatorname{FGM}_{\mathrm{I}(a=1 / b=0 / c / p)}$; (b) $\mathrm{FGM}_{\mathrm{II}(a=1 / b=0 / c / p)} ;$ (c) $\mathrm{FGM}_{\mathrm{I}(a=1 / b=0.5 / c=2 / p)} ;$ (d) $\mathrm{FGM}_{\mathrm{II}(a=1 / b=0.5 / c=2 / p)} ;$ (e) $\mathrm{FGM}_{\mathrm{I}(a=0 / b=-0.5 / c=2 / p)} ;$ (f) $\mathrm{FGM}_{\mathrm{II}(a=0 / b=-0.5 / c=2 / p)}$; (g) $\operatorname{FGM}_{\mathrm{I}(a=1 / b=1 / c=4 / p)}$; (h) $\mathrm{FGM}_{\mathrm{II}(a=1 / b=1 / c=4 / p)}$. 


$$
\begin{aligned}
& +2 D_{12}\left(\frac{1}{A} \frac{\partial \psi_{\alpha}}{\partial \alpha}+\frac{\psi_{\beta}}{A B} \frac{\partial A}{\partial \beta}\right)\left(\frac{1}{B} \frac{\partial \psi_{\beta}}{\partial \beta}+\frac{\psi_{\alpha}}{A B} \frac{\partial B}{\partial \alpha}\right) \\
& \left.+D_{66}\left(\frac{B}{A} \frac{\partial}{\partial \alpha}\left(\frac{\psi_{\beta}}{B}\right)+\frac{A}{B} \frac{\partial}{\partial \beta}\left(\frac{\psi_{\alpha}}{A}\right)\right)^{2}\right\} d S .
\end{aligned}
$$

The corresponding kinetic energy $(T)$ function of the moderately thick FG panels and shells of revolution can be given as

$$
\begin{aligned}
T & =\frac{1}{2} \iint_{S}\left\{I_{0}\left[\left(\frac{\partial u_{0}}{\partial t}\right)^{2}+\left(\frac{\partial v_{0}}{\partial t}\right)^{2}+\left(\frac{\partial w_{0}}{\partial t}\right)^{2}\right]\right. \\
& +2 I_{1}\left(\frac{\partial u_{0}}{\partial t} \frac{\partial \psi_{\alpha}}{\partial t}+\frac{\partial v_{0}}{\partial t} \frac{\partial \psi_{\beta}}{\partial t}\right) \\
& \left.+I_{2}\left[\left(\frac{\partial \psi_{\alpha}}{\partial t}\right)^{2}+\left(\frac{\partial \psi_{\beta}}{\partial t}\right)^{2}\right]\right\} d S,
\end{aligned}
$$

where

$$
\left(\begin{array}{lll}
I_{0} & I_{1} & I_{2}
\end{array}\right)=\int_{-h / 2}^{h / 2} \rho(z)\left(1, z^{1}, z^{2}\right) d z
$$

Since the main focus of this paper is to develop a unified computational model to study the vibration of moderately thick FG panels and shells of revolution with general boundary conditions, in order to satisfy the request, the artificial spring boundary technique is adopted here. In this technique, five groups of boundary restraining springs are arranged at all sides of the FG panels and shells of revolution to separately simulate the general boundary conditions. Then the equations describing general elastic supported moderately thick FG panels and shells of revolution can be written as follows:

$$
\begin{aligned}
k_{\alpha 0}^{u} u_{0} & =N_{\alpha}, \\
k_{\alpha 0}^{v} v_{0} & =N_{\alpha \beta}, \\
k_{\alpha 0}^{w} w_{0} & =Q_{\alpha}, \\
K_{\alpha 0}^{\alpha} \psi_{\alpha} & =M_{\alpha}, \\
K_{\alpha 0}^{\beta} \psi_{\beta} & =M_{\alpha \beta} \\
k_{\alpha 1}^{u} u_{0} & =-N_{\alpha}, \\
k_{\alpha 1}^{v} v_{0} & =-N_{\alpha \beta}, \\
k_{\alpha 1}^{w} w_{0} & =-Q_{\alpha}, \\
K_{\alpha 1}^{\alpha} \psi_{\alpha} & =-M_{\alpha}, \\
K_{\alpha 1}^{\beta} \psi_{\beta} & =-M_{\alpha \beta}
\end{aligned}
$$

$$
\begin{aligned}
k_{\beta 0}^{u} u_{0} & =N_{\beta}, \\
k_{\beta 0}^{u} v_{0} & =N_{\alpha \beta}, \\
k_{\beta 0}^{w} w_{0} & =Q_{\beta}, \\
K_{\beta 0}^{\alpha} \psi_{\alpha} & =M_{\beta}, \\
K_{\beta 0}^{\beta} \psi_{\beta} & =M_{\alpha \beta} \\
k_{\beta 1}^{u} u_{0} & =-N_{\beta}, \\
k_{\beta 1}^{u} v_{0} & =-N_{\alpha \beta}, \\
k_{\beta 1}^{w} w_{0} & =-Q_{\beta}, \\
K_{\beta 1}^{\alpha} \psi_{\alpha} & =-M_{\beta}, \\
K_{\beta 1}^{\beta} \psi_{\beta} & =-M_{\alpha \beta}
\end{aligned}
$$

Thus, as one of the merits of the present study, the unified treatment in dealing with the panels under general boundary conditions can be achieved by assigning the stiffness of the boundary springs with various values. For example, the free boundary condition can be readily obtained by setting the spring coefficients to zero and the clamped boundary can be obtained by assigning the springs' stiffness to infinity. Table 1 gives the corresponding spring stiffness values for the considered boundaries in which the symbol $D$ represents the bending stiffness $D=E_{c} h^{3} / 12\left(1-\mu_{c}{ }^{2}\right)$. Therefore, the potential energy $U_{\text {sp }}$ stored in the boundary springs is given as

$$
\begin{aligned}
U_{\mathrm{sp}} & =\frac{1}{2} \\
& \cdot \int\left\{\begin{array}{l}
{\left[k_{\alpha 0}^{u} u_{0}^{2}+k_{\alpha 0}^{v} v_{0}^{2}+k_{\alpha 0}^{w} w_{0}^{2}+K_{\alpha 0}^{\alpha} \psi_{\alpha}^{2}+K_{\alpha 0}^{\beta} \psi_{\beta}^{2}\right]_{\alpha=0}} \\
{\left[k_{\alpha 1}^{u} u_{0}^{2}+k_{\alpha 1}^{v} v_{0}^{2}+k_{\alpha 1}^{w} w_{0}^{2}+K_{\alpha 1}^{\alpha} \psi_{\alpha}^{2}+K_{\alpha 1}^{\beta} \psi_{\beta}^{2}\right]_{\alpha=L_{\alpha}}}
\end{array}\right\} B d \beta \\
& +\frac{1}{2} \\
& \cdot \int\left\{\begin{array}{l}
{\left[k_{\beta 0}^{u} u_{0}^{2}+k_{\beta 0}^{v} v_{0}^{2}+k_{\beta 0}^{w} w_{0}^{2}+K_{\beta 0}^{\alpha} \psi_{\alpha}^{2}+K_{\beta 0}^{\beta} \psi_{\beta}^{2}\right]_{\beta=0}} \\
{\left[k_{\beta 1}^{u} u_{0}^{2}+k_{\beta 1}^{v} v_{0}^{2}+k_{\beta 1}^{w} w_{0}^{2}+K_{\beta 1}^{\alpha} \psi_{\alpha}^{2}+K_{\beta 1}^{\beta} \psi_{\beta}^{2}\right]_{\beta=L_{\beta}}}
\end{array}\right\} A d \alpha .
\end{aligned}
$$

As mentioned before, we can know that the main focus and merits of this paper are to present a unified computational model of the functionally graded cylindrical, conical, spherical panels and shells of revolution. However, the previous studies are most confined to the signer geometric configuration, that is, panels and shells, the difficulty of which is that the admissible functions of the panels do not fit to the shells. As we all know, in addition to the external boundary conditions, the kinematic and physical compatibility should be satisfied at the common meridian of $\beta=0$ and $2 \pi$, if a complete shell of revolution needs considering [3, 53-55]. The kinematic compatibility conditions include the continuity 
TABLE 1: The corresponding spring stiffness values for general boundary conditions.

\begin{tabular}{|c|c|c|c|c|c|c|c|}
\hline \multirow{2}{*}{ Edges } & \multirow{2}{*}{$\mathrm{BC}$} & \multirow{2}{*}{ Essential conditions } & \multicolumn{5}{|c|}{ Corresponding spring stiffness values } \\
\hline & & & $\Gamma_{u}$ & $\Gamma_{v}$ & $\Gamma_{w}$ & $\Gamma_{x}$ & $\Gamma_{\theta}$ \\
\hline \multirow{6}{*}{$x=$ constant } & $\mathrm{F}$ & $N_{\alpha}=N_{\alpha \beta}=Q_{\alpha}=M_{\alpha}=M_{\alpha \beta}=0$ & 0 & 0 & 0 & 0 & 0 \\
\hline & $\mathrm{C}$ & $u_{0}=v_{0}=w_{0}=\psi_{\alpha}=\psi_{\beta}=0$ & $10^{8} \mathrm{D}$ & $10^{8} \mathrm{D}$ & $10^{8} \mathrm{D}$ & $10^{8} \mathrm{D}$ & $10^{8} \mathrm{D}$ \\
\hline & S & $u_{0}=v_{0}=w_{0}=M_{\alpha}=\psi_{\beta}=0$ & $10^{8} \mathrm{D}$ & $10^{8} \mathrm{D}$ & $10^{8} \mathrm{D}$ & 0 & $10^{8} \mathrm{D}$ \\
\hline & $E^{1}$ & $u_{0} \neq 0 ; v_{0}=w_{0}=\psi_{\alpha}=\psi_{\beta}=0$ & $10^{2} \mathrm{D}$ & $10^{2} \mathrm{D}$ & $10^{8} \mathrm{D}$ & $10^{8} \mathrm{D}$ & $10^{8} \mathrm{D}$ \\
\hline & $E^{2}$ & $v_{0} \neq 0 ; u_{0}=w_{0}=\psi_{\alpha}=\psi_{\beta}=0$ & $10^{8} \mathrm{D}$ & $10^{8} \mathrm{D}$ & $10^{2} \mathrm{D}$ & $10^{8} \mathrm{D}$ & $10^{8} \mathrm{D}$ \\
\hline & $\mathrm{E}^{3}$ & $u_{0} \neq 0, v_{0} \neq 0 ; w_{0}=\psi_{\alpha}=\psi_{\beta}=0$ & $10^{8} \mathrm{D}$ & $10^{8} \mathrm{D}$ & $10^{8} \mathrm{D}$ & $10^{2} \mathrm{D}$ & $10^{2} \mathrm{D}$ \\
\hline \multirow{6}{*}{$\theta=$ constant } & $\mathrm{F}$ & $N_{\beta}=N_{\alpha \beta}=Q_{\beta}=M_{\beta}=M_{\alpha \beta}=0$ & 0 & 0 & 0 & 0 & 0 \\
\hline & $\mathrm{C}$ & $u_{0}=v_{0}=w_{0}=\psi_{\alpha}=\psi_{\beta}=0$ & $10^{8} \mathrm{D}$ & $10^{8} \mathrm{D}$ & $10^{8} \mathrm{D}$ & $10^{8} \mathrm{D}$ & $10^{8} \mathrm{D}$ \\
\hline & S & $u_{0}=v_{0}=w_{0}=\psi_{\alpha}=M_{\beta}=0$ & $10^{8} \mathrm{D}$ & $10^{8} \mathrm{D}$ & $10^{8} \mathrm{D}$ & $10^{8} \mathrm{D}$ & 0 \\
\hline & $E^{1}$ & $u_{0} \neq 0 ; v_{0}=w_{0}=\psi_{\alpha}=\psi_{\beta}=0$ & $10^{2} \mathrm{D}$ & $10^{2} \mathrm{D}$ & $10^{8} \mathrm{D}$ & $10^{8} \mathrm{D}$ & $10^{8} \mathrm{D}$ \\
\hline & $\mathrm{E}^{2}$ & $v_{0} \neq 0 ; u_{0}=w_{0}=\psi_{\alpha}=\psi_{\beta}=0$ & $10^{8} \mathrm{D}$ & $10^{8} \mathrm{D}$ & $10^{2} \mathrm{D}$ & $10^{8} \mathrm{D}$ & $10^{8} \mathrm{D}$ \\
\hline & $\mathrm{E}^{3}$ & $u_{0} \neq 0, v_{0} \neq 0 ; w_{0}=\psi_{\alpha}=\psi_{\beta}=0$ & $10^{8} \mathrm{D}$ & $10^{8} \mathrm{D}$ & $10^{8} \mathrm{D}$ & $10^{2} \mathrm{D}$ & $10^{2} \mathrm{D}$ \\
\hline
\end{tabular}

of displacements. The physical compatibility conditions can only be the five continuous conditions for the generalized stress resultants. Thus, to consider the complete shell of revolution characterized by $\phi=2 \pi$, it is necessary to implement the kinematic and physical compatibility conditions between the two computational meridians of $\beta=0$ and $2 \pi$.

The kinematic compatibility conditions are as follows:

$$
\begin{gathered}
u_{0}(\alpha, 0, t)=u_{0}(\alpha, 2 \pi, t), \\
v_{0}(\alpha, 0, t)=v_{0}(\alpha, 2 \pi, t), \\
w_{0}(\alpha, 0, t)=w_{0}(\alpha, 2 \pi, t) \\
\psi_{\alpha}(\alpha, 0, t)=\psi_{\alpha}(\alpha, 2 \pi, t), \\
\psi_{\beta}(\alpha, 0, t)=\psi_{\beta}(\alpha, 2 \pi, t) .
\end{gathered}
$$

The physical compatibility conditions are as follows:

$$
\begin{gathered}
N_{\beta}(\alpha, 0, t)=N_{\beta}(\alpha, 2 \pi, t), \\
N_{\alpha \beta}(\alpha, 0, t)=N_{\alpha \beta}(\alpha, 2 \pi, t), \\
Q_{\beta}(\alpha, 0, t)=Q_{\beta}(\alpha, 2 \pi, t) \\
M_{\beta}(\alpha, 0, t)=M_{\beta}(\alpha, 2 \pi, t), \\
M_{\alpha \beta}(\alpha, 0, t)=M_{\alpha \beta}(\alpha, 2 \pi, t) .
\end{gathered}
$$

In order to achieve the above requirements, the authors draw on the experience of two elastically coupled rectangular plates [56] to present a coupling spring technique. As one innovation point of present study, three groups of linear and two groups of coupling springs are applied to imitate the kinematic compatibility and physical compatibility conditions of functionally graded panels at the common meridian of $\beta=0$ and $2 \pi$ when a complete shell of revolution is considered.
Therefore, the potential energies $\left(U_{c p}\right)$ stored in the five types of coupling springs can be defined as follows:

$$
\begin{aligned}
U_{\mathrm{cp}} & =\frac{1}{2} \int_{-h / 2}^{h / 2} \int_{0}^{L_{\alpha}}\left\{k_{u c}^{u}\left(\left.u_{0}\right|_{\beta=0}-\left.u_{0}\right|_{\beta=2 \pi}\right)^{2}\right. \\
& +k_{v c}^{v}\left(\left.v_{0}\right|_{\beta=0}-\left.v_{0}\right|_{\beta=2 \pi}\right)^{2} \\
& +k_{w c}^{w}\left(\left.w_{0}\right|_{\beta=0}-\left.w_{0}\right|_{\beta=2 \pi}\right)^{2} \\
& +K_{\alpha c}^{\alpha}\left(\left.\psi_{\alpha}\right|_{\beta=0}-\left.\psi_{\alpha}\right|_{\beta=2 \pi}\right)^{2} \\
& \left.+K_{\beta c}^{\beta}\left(\left.\psi_{\beta}\right|_{\beta=0}-\left.\psi_{\beta}\right|_{\beta=2 \pi}\right)^{2}\right\} B d \alpha d z .
\end{aligned}
$$

It should be noted that the stiffness of corresponding springs used at the common meridian of $\beta=0$ and $2 \pi$ of functionally graded panels is revalued to be zero automatically.

2.3. The Modified Fourier-Ritz Approach. The modified Fourier-Ritz approach was proposed by Jin et al. [49] to solve the free vibrations of laminated functionally graded shallow shells with general boundary conditions. The presented method consists of two main steps: firstly, the admissible functions of the structure elements are expanded into the modified Fourier series which consist of the two-dimensional (2D) Fourier cosine series and auxiliary functions to eliminate all the relevant discontinuities of the displacements and their derivatives at the edges regardless of boundary conditions; secondly, the natural frequencies of the structure elements are obtained by using the variational operation (Ritz method) in terms of the unknown the expanded coefficient. In contrast to most existing techniques, the modified FourierRitz method can be universally applicable to a variety of boundary conditions including all the classical cases, elastic restraints, and their combinations without the need of making any changes to the solution procedure. Therefore, each of the referenced surface displacements or rotations of the 
normal of a shell can be written as an improved Fourier series as follows:

$$
\begin{aligned}
u_{0}(\alpha, \beta, t)=\left(\sum_{m=0}^{\infty} \sum_{n=0}^{\infty} A_{m n}^{u} \cos \lambda_{m} \alpha \cos \lambda_{n} \beta\right. \\
+\sum_{l=1}^{2} \chi_{l}(\beta) \sum_{m=0}^{\infty} a_{m}^{l} \cos \lambda_{m} \alpha \\
\left.+\sum_{l=1}^{2} \zeta_{l}(\alpha) \sum_{n=0}^{\infty} b_{n}^{l} \cos \lambda_{n} \beta\right) e^{j \omega t} \\
+\psi_{\beta}(\alpha, \beta, t)=\left(\sum_{m=0}^{\infty} \sum_{n=0}^{\infty} B_{m n}^{v} \cos \lambda_{m} \alpha \cos \lambda_{n} \beta\right. \\
\left.+\sum_{l=1}^{2} \zeta_{l}(\alpha) \sum_{n=0}^{\infty} q_{n}^{l} \cos \lambda_{n} \beta\right) e^{j \omega t} \\
+\sum_{l=1}^{2} \chi_{l}(\beta) \sum_{m=0}^{\infty} c_{m}^{l} \cos \lambda_{m} \alpha \\
\left.+\sum_{l=1}^{2} \zeta_{l}(\alpha) \sum_{n=0}^{\infty} h_{n}^{l} \cos \lambda_{n} \beta\right) e^{j \omega t} \sum_{l=0}^{\infty} \sum_{n=0}^{\infty} k_{m}^{l} \cos \lambda_{m}^{\beta} \alpha \\
\left.+\sum_{n=0}^{\infty} d_{n}^{l} \cos \lambda_{n} \beta\right) e^{j \omega t} \\
\left.+\sum_{l=1}^{2} \zeta_{l}(\alpha) \sum_{n=0}^{\infty} f_{n}^{l} \cos \lambda_{m} \beta\right) e^{j \omega t} \sum_{m=0}^{\infty} g_{m}^{l} \cos \lambda_{m} \alpha \\
+\left(\sum_{m}^{l} \cos \lambda_{m} \alpha\right. \\
w_{0}(\alpha, t)=\left(\sum_{m=0}^{\infty} \sum_{n=0}^{\infty} C_{m n}^{w} \cos \lambda_{m} \alpha \cos \lambda_{n} \beta\right.
\end{aligned}
$$

where $\lambda_{m}=m \pi / L_{\alpha}, \lambda_{n}=n \pi / L_{\beta}$, and $A_{m n}^{u}, B_{m n}^{v}, C_{m n}^{w}$, $D_{m n}^{\varphi}$, and $E_{m n}^{\theta}$ are the Fourier coefficients of two-dimensional Fourier series expansions for the displacements functions, respectively. $a_{m}^{l}, b_{n}^{l}, c_{m}^{l}, d_{n}^{l}, e_{m}^{l}, f_{n}^{l}, g_{m}^{l}, h_{n}^{l}, k_{m}^{l}$, and $q_{n}^{l}$ are the supplemented coefficients of the auxiliary functions $\chi_{l}(\beta)$ and $\zeta_{l}(\alpha)$, where $l=1,2$. The two types of auxiliary functions $\chi_{l}(\beta)$ and $\zeta_{l}(\alpha)$ are selected to remove all the discontinuities potentially associated with the first-order derivatives at the boundaries. Thus, the function sets are capable of representing any free vibration motion of the shell. The two types of auxiliary functions are given as

$$
\begin{aligned}
& \zeta(\alpha) \\
& =\left\{\begin{array}{l}
\zeta_{1}(\varphi)=\frac{L_{\alpha}}{2 \pi} \sin \left(\frac{\pi \alpha}{2 L_{\alpha}}\right)+\frac{L_{\alpha}}{2 \pi} \sin \left(\frac{3 \pi \alpha}{2 L_{\alpha}}\right) \\
\zeta_{2}(\varphi)=-\frac{L_{\alpha}}{2 \pi} \cos \left(\frac{\pi \alpha}{2 L_{\alpha}}\right)+\frac{L_{\alpha}}{2 \pi} \cos \left(\frac{3 \pi \alpha}{2 L_{\alpha}}\right)
\end{array}\right.
\end{aligned}
$$

$$
\begin{aligned}
& \chi(\beta) \\
& =\left\{\begin{array}{l}
\chi_{1}(\beta)=\frac{L_{\beta}}{2 \pi} \sin \left(\frac{\pi \beta}{2 L_{\beta}}\right)+\frac{L_{\beta}}{2 \pi} \sin \left(\frac{3 \pi \beta}{2 L_{\beta}}\right) \\
\chi_{2}(\beta)=-\frac{L_{\beta}}{2 \pi} \cos \left(\frac{\pi \beta}{2 L_{\beta}}\right)+\frac{L_{\beta}}{2 \pi} \cos \left(\frac{3 \pi \beta}{2 L_{\beta}}\right) .
\end{array}\right.
\end{aligned}
$$

It is easy to verify that

$$
\begin{aligned}
& \zeta_{1}(0)=\zeta_{1}\left(L_{\alpha}\right)=\zeta_{1}^{\prime}\left(L_{\alpha}\right)=0, \quad \zeta_{1}^{\prime}(0)=1 \\
& \zeta_{2}(0)=\zeta_{2}\left(L_{\alpha}\right)=\zeta_{2}^{\prime}(0)=0, \quad \zeta_{2}^{\prime}\left(L_{\alpha}\right)=1 \\
& \chi_{1}(0)=\chi_{1}\left(L_{\beta}\right)=\chi_{1}^{\prime}\left(L_{\beta}\right)=0, \quad \chi_{1}^{\prime}(0)=1 \\
& \chi_{2}(0)=\chi_{2}\left(L_{\beta}\right)=\chi_{2}^{\prime}(0)=0, \quad \chi_{2}^{\prime}\left(L_{\beta}\right)=1
\end{aligned}
$$

The Lagrangian energy function $(L)$ of the moderately thick functionally graded panels and shells of revolution can be written as

$$
L=T-U_{s}-U_{\mathrm{sp}}-U_{\mathrm{cp}} .
$$

Substituting (9), (11), (14), and (17) into (21), then the Lagrangian expression is minimized by taking its derivatives with respect to these coefficients:

$$
\begin{aligned}
& \frac{\partial L}{\partial \iota}=0, \\
& \quad \iota=A_{m n}^{u}, a_{m}^{l}, b_{n}^{l}, B_{m n}^{v}, c_{m}^{l}, d_{n}^{l}, \ldots, E_{m n}^{\beta}, g_{m}^{l}, h_{n}^{l} .
\end{aligned}
$$

Since the displacements and rotation components of the moderately thick functionally graded panels and shells of revolution are chosen as $\mathbf{M}$ and $\mathbf{N}$ to obtain the results with acceptable accuracy, a total of $5 \times(\mathbf{M}+1) \times(\mathbf{N}+1)+10 \times$ $(\mathbf{M}+\mathbf{N}+2)$ equations are obtained. They can be summed up in a matrix form:

$$
\left(\mathbf{K}-\omega^{2} \mathbf{M}\right) \mathbf{H}=\mathbf{0},
$$

where $\mathbf{K}, \mathbf{M}$, and $\mathbf{H}$, respectively, represent the stiffness matrix, mass matrix, and vector of the unknown coefficients for the panel and shell. The detailed expressions for above matrices are given in Appendix. By solving (23), the frequencies (or eigenvalues) of moderately thick functionally graded panels and shells of revolution can be readily obtained and the mode shapes can be yielded by substituting the corresponding eigenvectors into series representations of displacement components. 
TABLE 2: Convergence of frequency parameters of the single curved shells with different coupling parameters $\varkappa$.

\begin{tabular}{lccccccccccc}
\hline Shape & Mode & & & & & & & & & \\
& & 1 & $10^{3}$ & $10^{5}$ & $10^{7}$ & $10^{8}$ & $10^{10}$ & $10^{12}$ & $10^{14}$ & $10^{16}$ \\
\hline & 1 & 10.043 & 10.043 & 10.058 & 10.680 & 11.659 & 16.469 & 16.779 & 16.780 & 16.780 & 16.780 \\
& 2 & 10.051 & 10.051 & 10.062 & 11.315 & 14.581 & 16.681 & 16.779 & 16.780 & 16.780 & 16.780 \\
Cylindrical shells & 3 & 16.515 & 16.515 & 16.518 & 16.685 & 16.857 & 17.399 & 17.711 & 17.714 & 17.714 & 17.714 \\
& 4 & 17.203 & 17.203 & 17.204 & 17.215 & 17.236 & 17.399 & 17.711 & 17.714 & 17.714 & 17.714 \\
& 5 & 17.564 & 17.564 & 17.566 & 17.744 & 19.153 & 21.296 & 21.381 & 21.381 & 21.381 & 21.381 \\
& 6 & 19.644 & 19.644 & 19.645 & 19.685 & 19.762 & 21.374 & 21.381 & 21.381 & 21.381 & 21.381 \\
& 1 & 10.376 & 10.376 & 10.391 & 11.164 & 12.259 & 17.946 & 18.161 & 18.162 & 18.162 & 18.162 \\
Conical shells & 2 & 10.392 & 10.392 & 10.418 & 12.431 & 16.393 & 17.985 & 18.161 & 18.162 & 18.162 & 18.162 \\
& 3 & 17.786 & 17.786 & 17.790 & 17.990 & 18.164 & 18.915 & 19.280 & 19.286 & 19.286 & 19.286 \\
& 4 & 18.553 & 18.553 & 18.557 & 18.676 & 18.693 & 18.994 & 19.280 & 19.286 & 19.286 & 19.286 \\
& 5 & 18.725 & 18.725 & 18.726 & 19.009 & 20.736 & 21.794 & 21.855 & 21.855 & 21.855 & 21.855 \\
& 6 & 20.595 & 20.595 & 20.602 & 21.018 & 21.415 & 21.802 & 21.855 & 21.855 & 21.855 & 21.855 \\
& 1 & 9.766 & 9.761 & 9.757 & 9.867 & 10.088 & 11.761 & 12.034 & 12.038 & 12.038 & 12.038 \\
& 2 & 9.766 & 9.764 & 9.769 & 10.017 & 10.809 & 11.827 & 12.036 & 12.038 & 12.038 & 12.038 \\
& 2 & 12.046 & 12.046 & 12.046 & 12.055 & 12.086 & 12.163 & 12.221 & 12.224 & 12.224 & 12.224 \\
& 3 & 12.117 & 12.117 & 12.118 & 12.133 & 12.153 & 12.185 & 12.223 & 12.224 & 12.224 & 12.224 \\
& 4 & 12.308 & 12.308 & 12.319 & 12.321 & 12.340 & 12.672 & 13.084 & 13.089 & 13.089 & 13.089 \\
& 5 & 12.493 & 12.493 & 12.493 & 12.523 & 12.665 & 12.947 & 13.085 & 13.089 & 13.089 & 13.089 \\
\hline
\end{tabular}

\section{Numerical Results and Discussion}

In this section, some results and discussions about the free vibration of four-parameter functionally graded moderately thick cylindrical, conical, spherical panels and shells of revolution are presented to verify the accuracy and flexibility of the proposed unified approach. Unless mentioned otherwise, the panels and shells under consideration are assumed to be made of a mixture of $\mathrm{ZnO}_{2}\left(E_{c}=168 \mathrm{GPa}, \mu_{c}=0.3\right.$, $\left.\rho_{c}=5700 \mathrm{~kg} / \mathrm{m}^{3}\right)$ and $\mathrm{Al}\left(E_{m}=70 \mathrm{GPa}, \mu_{m}=0.3\right.$, $\left.\rho_{m}=2707 \mathrm{~kg} / \mathrm{m}^{3}\right)$. In the following frequencies results, the nondimensional frequency parameters of the cylindrical, conical, and spherical panels and shells are expressed as follows: cylindrical meridian: $\Omega=\omega L^{2} / h \sqrt{\rho_{c} / E_{c}}$; conical meridian: $\Omega=\omega L_{0}^{2} / h \sqrt{\rho_{c} / E_{c}}$; spherical meridian: $\Omega=$ $\omega R^{2} / h \sqrt{\rho_{c} / E_{c}}$.

3.1. Convergence Study and Formulation Validation. From the theoretical formulations, we can know that the computational accuracy relies on a limited number of terms in the displacement expressions in actual calculation. In addition, when considering the shells of revolution, the results strongly rely on the coupling parameters. Thus, one important issue is the choice of the appropriate truncation terms and coupling parameters. The nondimensional frequency parameter convergence of the clamped shells with respect to different coupling parameters is examined in Table 2. The geometrical parameters and power-law exponents for the functionally graded shells used in the study are as follows: cylindrical shell: $R=1 \mathrm{~m}, L=4 \mathrm{~m}, h=0.1 \mathrm{~m}$, and $p=0$; conical shell: $R_{1}=0.5 \mathrm{~m}, L_{0}=4 / \cos \left(\alpha_{0}\right) R_{1}, h=0.1 \mathrm{~m}, \alpha_{0}=40^{\circ}$, and $p=0$; spherical shell: $R=1 \mathrm{~m}, \varphi_{0}=\pi / 6, \varphi_{0}=\pi / 2$, $h=0.1 \mathrm{~m}$, and $p=0$. All coupling parameters are defined with the same value and defined as follows: $k_{u c}^{u}=k_{v c}^{v}=k_{w c}^{w}=$ $K_{\alpha c}^{\alpha}=K_{\beta c}^{\beta}=\gamma$. It is obvious that the frequency parameters rapidly converge as the coupling parameter increases. In the absence of the coupling parameters or for small coupling parameters, pseudovibration characteristic may appear in the present solution. This implies that small coupling parameters cannot impose the interface continuity conditions in a proper manner. Thus, based on the existed analysis, $x=10^{14}$ has been adopted to present all the results in the following analysis.

Next, the convergence studies of the cylindrical, conical, and spherical panels and shells will be discussed. Figure 5 shows the convergence studies of the first three frequency parameters for the structure elements for complete clamped FG panels and shells with different truncated numbers $M$ and $N$. The geometrical dimensions of the shells are the same as Table 2 and the panels are also in accordance with Table 2 except the circumferential angle $\phi=120^{\circ}$. From the figure, we can know that the proposed unified approach has fast convergence and good stability. In view of the excellent numerical behavior of the current solution, the truncation numbers will be simply set as $M=N=15$ in the following calculations.

The validation of the unified computational model versus about the different geometric parameters is shown in Tables $3-8$. The details regarding the geometry of the structures considered are indicated as follows:

(1) Cylindrical panels (FFCF): $R=1 \mathrm{~m}, L=2 \mathrm{~m}, h=$ $0.1 \mathrm{~m}$, and $\phi=120^{\circ}$ (Table 3).

(2) Cylindrical shells (FC): $R=1 \mathrm{~m}, L=2 \mathrm{~m}$, and $h=$ $0.1 \mathrm{~m}$ (Table 4). 

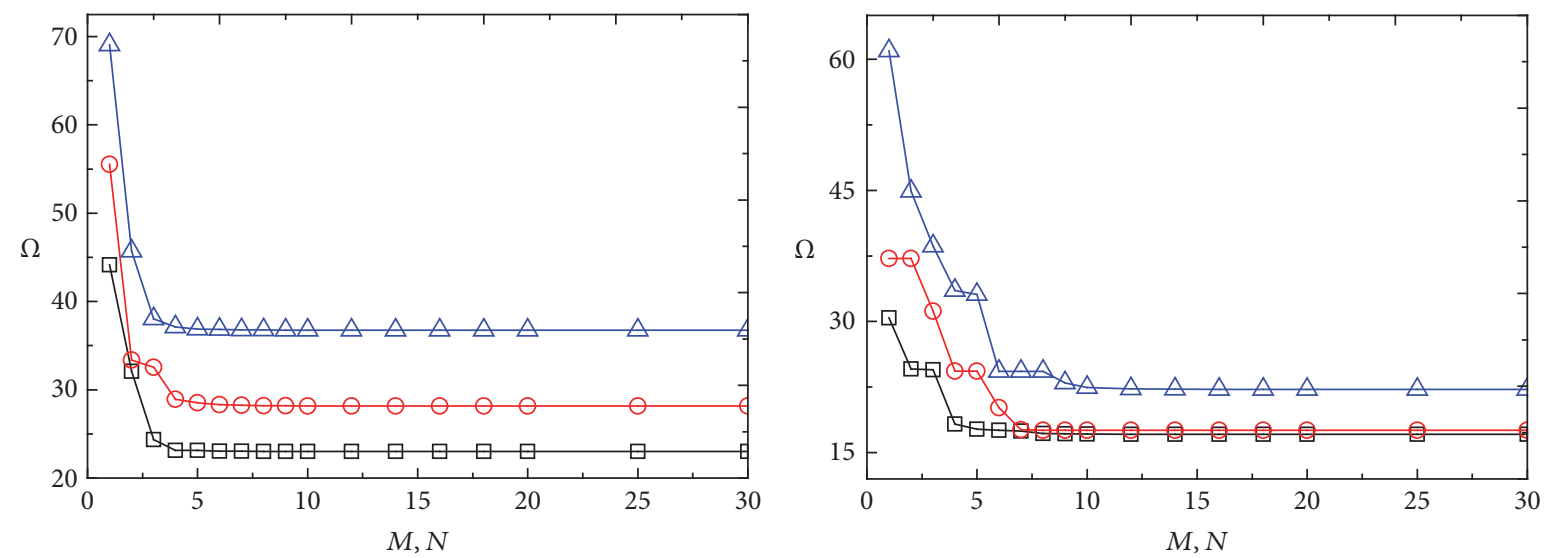

$\square$ 1st mode

- 2nd mode

$\square$ 1st mode

- 2nd mode

$\triangle$ 3rd mode

(a)
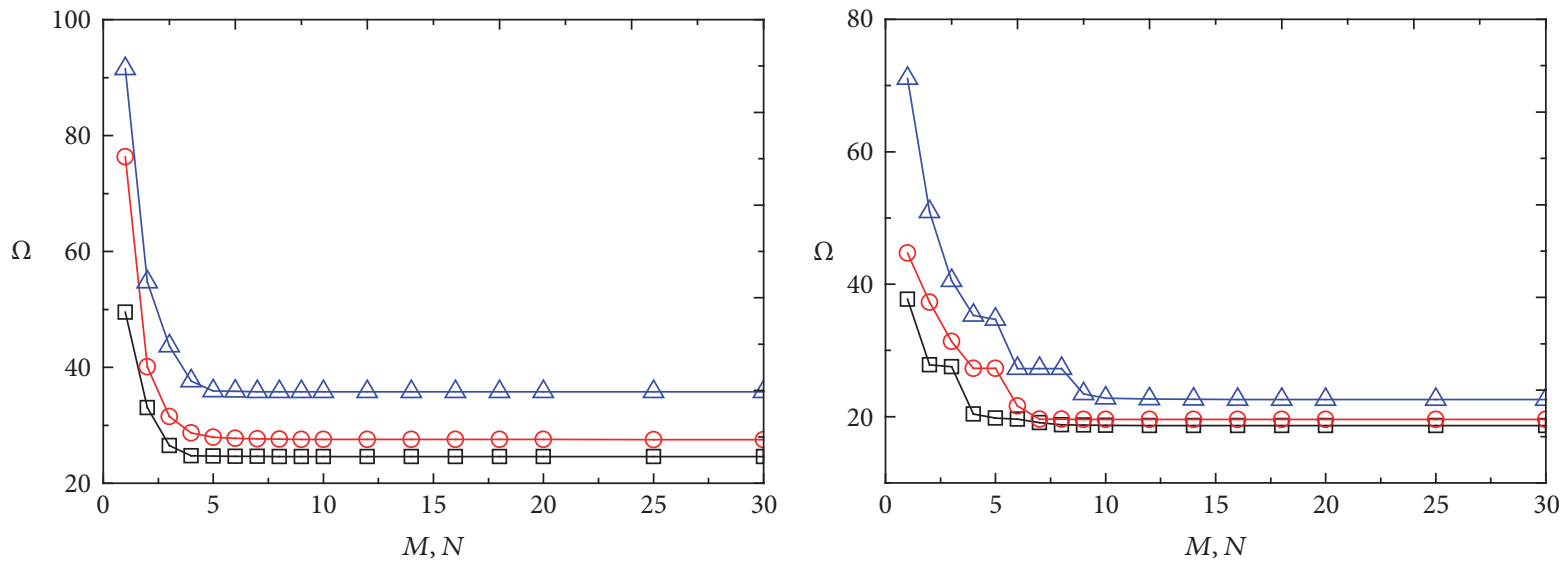

$\square$ 1st mode

- 2 nd mode

$\triangle$ 3rd mode

$\square 1$ st mode

- 2nd mode

$\triangle$ 3rd mode

(d)
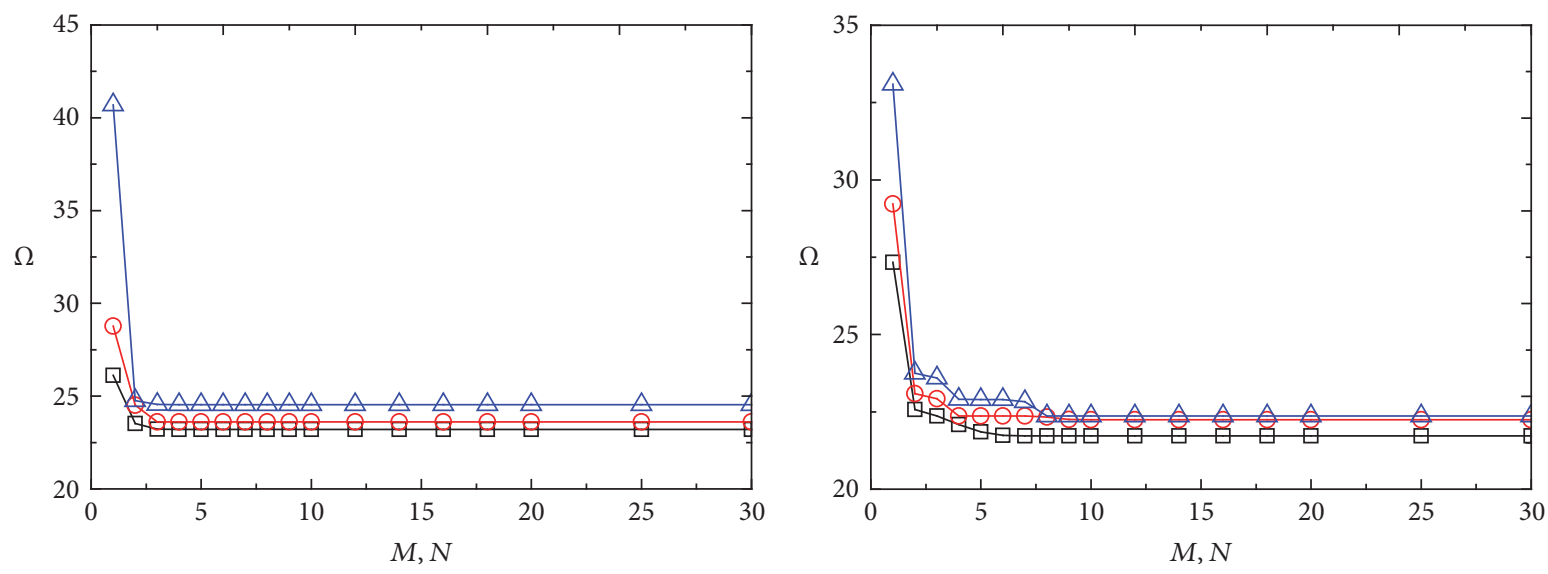

$$
\begin{aligned}
& \square \text { 1st mode } \\
& - \text { 2nd mode } \\
& \triangle \text { 3rd mode }
\end{aligned}
$$$$
\begin{aligned}
& \square-1 \text { st mode } \\
& - \text { 2nd mode } \\
& \triangle \text { 3rd mode }
\end{aligned}
$$

(e)

(f)

FIGURE 5: Variation of the frequency parameters $\Omega$ versus the truncated numbers $M$ and $N$ for complete clamped FG panels and shells: (a) cylindrical panels; (b) cylindrical shells; (c) conical panels; (d) conical shells; (e) spherical panels; (f) spherical shells. 
TABLE 3: Comparison of the first nine frequencies $(\mathrm{Hz})$ of functionally graded cylindrical panels with different power-law index $p$.

\begin{tabular}{|c|c|c|c|c|c|c|c|c|c|c|}
\hline \multirow{2}{*}{$p$} & \multirow{2}{*}{ Method } & \multicolumn{9}{|c|}{ Mode numbers } \\
\hline & & 1 & 2 & 3 & 4 & 5 & 6 & 7 & 8 & 9 \\
\hline \multirow{3}{*}{0} & Ref [52] & 61.02 & 94.82 & 153.13 & 241.39 & 275.83 & 291.45 & 355.14 & 450.76 & 511.92 \\
\hline & Ref [8] & 61.06 & 94.92 & 153.11 & 241.75 & 276.37 & 291.94 & 355.07 & 450.82 & 512.48 \\
\hline & Present & 61.02 & 94.82 & 153.12 & 241.60 & 276.22 & 291.64 & 355.09 & 450.72 & 512.31 \\
\hline \multirow{3}{*}{0.6} & $\operatorname{Ref}[52]$ & 59.24 & 93.14 & 148.51 & 234.79 & 267.06 & 287.85 & 343.22 & 443.06 & 495.89 \\
\hline & Ref [8] & 59.13 & 93.03 & 148.14 & 234.60 & 266.97 & 287.63 & 342.38 & 442.09 & 495.31 \\
\hline & Present & 59.09 & 92.93 & 148.15 & 234.45 & 266.83 & 287.34 & 342.39 & 441.99 & 495.15 \\
\hline \multirow{3}{*}{1} & $\operatorname{Ref}[52]$ & 58.96 & 92.65 & 147.94 & 233.64 & 265.86 & 286.16 & 342.08 & 440.72 & 493.67 \\
\hline & Ref [8] & 58.82 & 92.48 & 147.49 & 233.31 & 265.61 & 285.77 & 341.04 & 439.49 & 492.80 \\
\hline & Present & 58.78 & 92.38 & 147.50 & 233.16 & 265.47 & 285.48 & 341.05 & 439.39 & 492.63 \\
\hline \multirow{3}{*}{5} & $\operatorname{Ref}[52]$ & 60.09 & 91.69 & 152.02 & 236.75 & 272.42 & 279.34 & 354.89 & 436.34 & 505.35 \\
\hline & Ref [8] & 59.96 & 91.54 & 151.57 & 236.42 & 272.19 & 279.06 & 353.83 & 435.18 & 504.56 \\
\hline & Present & 59.92 & 91.44 & 151.59 & 236.29 & 272.04 & 278.78 & 353.84 & 435.09 & 504.37 \\
\hline \multirow{3}{*}{20} & Ref [52] & 59.19 & 90.29 & 149.49 & 233.27 & 268.37 & 275.23 & 348.80 & 429.63 & 497.87 \\
\hline & Ref [8] & 59.16 & 90.30 & 149.29 & 233.32 & 268.57 & 275.41 & 348.32 & 429.19 & 497.87 \\
\hline & Present & 59.12 & 90.20 & 149.30 & 233.20 & 268.43 & 275.13 & 348.33 & 429.10 & 497.69 \\
\hline \multirow{3}{*}{50} & $\operatorname{Ref}[52]$ & 58.20 & 89.55 & 146.55 & 229.79 & 263.58 & 273.98 & 341.04 & 425.83 & 489.06 \\
\hline & $\operatorname{Ref}[8]$ & 58.21 & 89.60 & 146.45 & 230.00 & 263.95 & 274.32 & 340.79 & 425.66 & 489.36 \\
\hline & Present & 58.17 & 89.51 & 146.46 & 229.87 & 263.81 & 274.04 & 340.80 & 425.57 & 489.19 \\
\hline
\end{tabular}

TABLE 4: Comparison of the first nine frequencies $(\mathrm{Hz})$ of functionally graded cylindrical shells with different power-law index $p$.

\begin{tabular}{|c|c|c|c|c|c|c|c|c|c|}
\hline \multirow{2}{*}{$p$} & \multirow{2}{*}{ Method } & \multicolumn{8}{|c|}{ Mode number } \\
\hline & & 1 & 2 & 3 & 4 & 5 & 6 & 7 & 8 \\
\hline \multirow{3}{*}{0} & $\operatorname{Ref}[1]$ & 152.93 & 152.93 & 220.06 & 220.06 & 253.78 & 253.78 & 383.55 & 383.56 \\
\hline & $\operatorname{Ref}[9]$ & 152.89 & 152.89 & 219.97 & 219.97 & 253.79 & 253.79 & 383.44 & 383.44 \\
\hline & Present & 152.89 & 152.89 & 220.12 & 220.12 & 253.77 & 253.77 & 383.95 & 383.95 \\
\hline \multirow{4}{*}{0.6} & $\operatorname{Ref}[1]$ & 152.25 & 152.25 & 219.86 & 219.86 & 252.17 & 252.17 & 383.39 & 383.40 \\
\hline & $\operatorname{Ref}[27]$ & 152.02 & 152.02 & 219.54 & 219.54 & 251.91 & 251.91 & 382.87 & 382.87 \\
\hline & Ref [9] & 152.07 & 152.07 & 219.59 & 219.59 & 251.96 & 251.96 & 382.95 & 382.95 \\
\hline & Present & 152.08 & 152.08 & 219.73 & 219.83 & 251.95 & 251.95 & 383.46 & 383.46 \\
\hline \multirow{3}{*}{1} & $\operatorname{Ref}[1]$ & 151.77 & 151.77 & 219.56 & 219.56 & 251.14 & 251.14 & 382.97 & 382.97 \\
\hline & Ref [9] & 151.52 & 151.52 & 219.19 & 219.19 & 250.81 & 250.81 & 382.35 & 382.35 \\
\hline & Present & 151.53 & 151.53 & 219.33 & 219.33 & 250.80 & 250.80 & 382.85 & 382.85 \\
\hline \multirow{4}{*}{5} & $\operatorname{Ref}[1]$ & 148.97 & 148.97 & 218.87 & 218.88 & 244.40 & 244.40 & 382.46 & 382.47 \\
\hline & Ref [27] & 148.50 & 148.50 & 218.16 & 218.16 & 243.73 & 243.73 & 381.26 & 381.26 \\
\hline & Ref [9] & 148.53 & 148.53 & 218.21 & 218.21 & 243.76 & 243.76 & 381.33 & 381.33 \\
\hline & Present & 148.54 & 148.54 & 218.35 & 218.35 & 243.75 & 243.75 & 381.82 & 381.82 \\
\hline \multirow{4}{*}{20} & $\operatorname{Ref}[1]$ & 146.46 & 146.46 & 215.90 & 215.90 & 239.84 & 239.84 & 377.34 & 377.34 \\
\hline & Ref [27] & 146.21 & 146.21 & 215.50 & 215.50 & 239.54 & 239.54 & 376.69 & 376.69 \\
\hline & Ref [9] & 146.24 & 146.24 & 215.55 & 215.55 & 239.57 & 239.57 & 376.76 & 376.76 \\
\hline & Present & 146.25 & 146.25 & 215.69 & 215.69 & 239.56 & 239.56 & 377.23 & 377.23 \\
\hline
\end{tabular}


TABLE 5: Comparison of the first nine frequencies $(\mathrm{Hz})$ of the functionally graded conical panels with different power-law index $p$.

\begin{tabular}{|c|c|c|c|c|c|c|c|c|c|c|}
\hline \multirow{2}{*}{$p$} & \multirow{2}{*}{ Method } & \multicolumn{9}{|c|}{ Mode number } \\
\hline & & 1 & 2 & 3 & 4 & 5 & 6 & 7 & 8 & 9 \\
\hline \multicolumn{11}{|c|}{$\operatorname{FGM}_{\mathrm{I}(a=1 / b=0.5 / c=2 / p)}$} \\
\hline \multirow{4}{*}{0.6} & $\operatorname{Ref}[3]$ & 80.10 & 110.93 & 158.60 & 195.20 & 259.10 & 273.52 & 321.39 & 360.77 & 396.83 \\
\hline & $\operatorname{Ref}[10]$ & 80.15 & 111.03 & 158.61 & 195.49 & 259.31 & 273.52 & 321.38 & 361.16 & 397.06 \\
\hline & Present & 80.14 & 110.95 & 158.62 & 195.57 & 259.18 & 273.62 & 321.42 & 361.38 & 397.12 \\
\hline & $\operatorname{Ref}[3]$ & 79.89 & 110.56 & 158.15 & 194.72 & 258.36 & 272.88 & 320.38 & 359.84 & 395.97 \\
\hline \multirow[t]{3}{*}{1} & $\operatorname{Ref}[10]$ & 79.91 & 110.62 & 158.10 & 194.94 & 258.48 & 272.82 & 320.28 & 360.10 & 396.11 \\
\hline & Present & 79.91 & 110.54 & 158.11 & 195.02 & 258.36 & 272.91 & 320.31 & 360.33 & 396.18 \\
\hline & Ref [3] & 78.92 & 108.45 & 156.07 & 192.86 & 254.58 & 270.76 & 315.52 & 356.30 & 393.68 \\
\hline \multirow[t]{3}{*}{5} & $\operatorname{Ref}[10]$ & 78.86 & 108.39 & 155.87 & 192.87 & 254.45 & 270.51 & 315.12 & 356.20 & 393.55 \\
\hline & Present & 78.86 & 108.31 & 155.88 & 192.95 & 254.33 & 270.60 & 315.16 & 356.42 & 393.62 \\
\hline & $\operatorname{Ref}[3]$ & 77.81 & 106.72 & 153.93 & 190.43 & 250.80 & 267.51 & 311.11 & 351.98 & 389.27 \\
\hline \multirow[t]{3}{*}{20} & $\operatorname{Ref}[10]$ & 77.83 & 106.78 & 153.90 & 190.65 & 250.92 & 267.46 & 311.03 & 352.24 & 389.42 \\
\hline & Present & 77.83 & 106.70 & 153.91 & 190.73 & 250.80 & 267.56 & 311.07 & 352.46 & 389.48 \\
\hline & Ref [3] & 76.57 & 105.58 & 151.56 & 187.03 & 247.32 & 262.39 & 306.75 & 345.73 & 381.29 \\
\hline \multirow[t]{2}{*}{50} & $\operatorname{Ref}[10]$ & 76.63 & 105.69 & 151.60 & 187.34 & 247.57 & 262.43 & 306.81 & 346.17 & 381.56 \\
\hline & Present & 76.63 & 105.62 & 151.61 & 187.43 & 247.45 & 262.53 & 306.85 & 346.39 & 381.63 \\
\hline \multicolumn{11}{|c|}{$\mathrm{FGM}_{\mathrm{II}(a=1 / b=0.5 / c=2 / p)}$} \\
\hline \multirow{4}{*}{0.6} & $\operatorname{Ref}[3]$ & 79.87 & 110.71 & 158.27 & 194.75 & 258.47 & 272.88 & 320.85 & 360.10 & 395.93 \\
\hline & $\operatorname{Ref}[10]$ & 80.03 & 110.96 & 158.48 & 195.29 & 258.99 & 273.14 & 321.24 & 360.94 & 396.49 \\
\hline & Present & 80.03 & 110.88 & 158.49 & 195.37 & 258.86 & 273.23 & 321.27 & 361.16 & 396.56 \\
\hline & Ref [3] & 79.54 & 110.22 & 157.64 & 194.02 & 257.39 & 271.89 & 319.56 & 358.81 & 394.58 \\
\hline \multirow[t]{3}{*}{1} & $\operatorname{Ref}[10]$ & 79.73 & 110.51 & 157.90 & 194.63 & 257.99 & 272.21 & 320.05 & 359.76 & 395.23 \\
\hline & Present & 79.73 & 110.43 & 157.91 & 194.71 & 257.86 & 272.31 & 320.08 & 359.98 & 395.30 \\
\hline & $\operatorname{Ref}[3]$ & 78.23 & 107.82 & 155.00 & 191.47 & 252.65 & 268.67 & 313.79 & 354.27 & 390.76 \\
\hline \multirow[t]{3}{*}{5} & $\operatorname{Ref}[10]$ & 78.49 & 108.21 & 155.40 & 192.25 & 253.46 & 269.17 & 314.54 & 355.53 & 391.65 \\
\hline & Present & 78.49 & 108.13 & 155.41 & 192.33 & 253.34 & 269.26 & 314.58 & 355.75 & 391.71 \\
\hline & Ref [3] & 77.49 & 106.44 & 153.43 & 189.81 & 249.93 & 266.54 & 310.31 & 351.07 & 387.92 \\
\hline \multirow[t]{3}{*}{20} & Ref [10] & 77.67 & 106.70 & 153.67 & 190.37 & 250.47 & 266.83 & 310.75 & 351.94 & 388.52 \\
\hline & Present & 77.66 & 106.63 & 153.68 & 190.45 & 250.35 & 266.92 & 310.78 & 352.16 & 388.59 \\
\hline & Ref [3] & 76.43 & 105.46 & 151.35 & 186.76 & 246.94 & 261.96 & 306.41 & 345.34 & 380.70 \\
\hline \multirow[t]{2}{*}{50} & $\operatorname{Ref}[10]$ & 76.56 & 105.66 & 151.50 & 187.22 & 247.36 & 262.15 & 306.69 & 346.04 & 381.16 \\
\hline & Present & 76.56 & 105.59 & 151.52 & 187.30 & 247.25 & 262.25 & 306.73 & 346.26 & 381.24 \\
\hline
\end{tabular}

(3) Conical panels (SCSC): $R_{1}=1 \mathrm{~m}, L_{0}=2 \mathrm{~m}, h=$ $0.1 \mathrm{~m}, \alpha_{0}=40^{\circ}$, and $\phi=120^{\circ}$ (Table 5).

(4) Conical shells (FC): $R_{1}=0.5 \mathrm{~m}, L_{0} \cos \alpha_{0}=2 \mathrm{~m}, h=$ $0.1 \mathrm{~m}$, and $\alpha_{0}=40^{\circ}$ (Table 6).

(5) Spherical panels (FFCF): $R=2 \mathrm{~m}, h=0.1 \mathrm{~m}, \varphi_{0}=$ $30^{\circ}, \varphi_{1}=90^{\circ}$, and $\phi=120^{\circ}$ (Table 7).

(6) Spherical shells: $R=1 \mathrm{~m}, h=0.05 \mathrm{~m}, \varphi_{0}=22.5^{\circ}$, and $\varphi_{1}=90^{\circ}$ (Table 8).

In order to check the present method, the numerical results reported by $\mathrm{Su}$ et al. [7-9], $\mathrm{Qu}$ et al. [27], and Tornabene et al. $[1,3,52]$ are also given in the above tables for comparison. From the comparisons, we can see a consistent agreement of present results taken from the current proposed unified approach and referential data. Besides, Tables 3-8 also show that it is appropriate to define the classical boundary conditions in terms of the boundary spring rigidities. Based on the formulation validation, some new results of the FG cylindrical, conical, and spherical panels and shells with classical-elastic and elastic restrain boundary condition are shown in Tables 9-14. Also, for any given modal frequency, the corresponding mode shape can be easily determined by submitting the solved eigenvector into (22). Thus, some select mode shapes of that are performed in Figures 6-8.

3.2. Parameter Studies. Although the above results of the FG cylindrical, conical, and spherical panels and shells with classical-elastic and elastic restrain boundary conditions can 
TABLE 6: Comparison of the first nine frequencies $(\mathrm{Hz})$ of the functionally graded conical shells with different power-law index $p$.

\begin{tabular}{|c|c|c|c|c|c|c|c|c|c|}
\hline \multirow{2}{*}{$p$} & \multirow{2}{*}{ Method } & \multicolumn{8}{|c|}{ Mode number } \\
\hline & & 1 & 2 & 3 & 4 & 5 & 6 & 7 & 8 \\
\hline \multicolumn{10}{|c|}{$\mathrm{FGM}_{\mathrm{I}(a=0 / b=-0.5 / c=2 / p)}$} \\
\hline \multirow{4}{*}{0.6} & Ref [1] & 208.92 & 208.92 & 230.11 & 230.11 & 284.73 & 284.74 & 321.51 & 321.51 \\
\hline & $\operatorname{Ref}[29]$ & 208.75 & 208.75 & 229.96 & 229.96 & 284.60 & 284.60 & 321.33 & 321.33 \\
\hline & Ref [11] & 208.58 & 208.58 & 230.06 & 230.06 & 284.67 & 284.67 & 321.28 & 321.28 \\
\hline & Present & 208.78 & 208.78 & 230.06 & 230.06 & 284.92 & 284.92 & 321.32 & 321.32 \\
\hline \multirow{4}{*}{5} & $\operatorname{Ref}[1]$ & 204.81 & 204.81 & 223.84 & 223.84 & 275.52 & 275.53 & 316.64 & 316.64 \\
\hline & Ref [29] & 204.25 & 204.25 & 223.36 & 223.36 & 275.02 & 275.02 & 315.94 & 315.94 \\
\hline & $\operatorname{Ref}[11]$ & 203.36 & 203.36 & 223.72 & 223.72 & 275.26 & 275.26 & 315.48 & 315.48 \\
\hline & Present & 204.28 & 204.28 & 223.46 & 223.46 & 275.35 & 275.35 & 315.93 & 315.93 \\
\hline \multirow{4}{*}{20} & Ref [1] & 204.89 & 204.89 & 227.33 & 227.33 & 282.68 & 282.69 & 312.50 & 312.50 \\
\hline & Ref [29] & 204.27 & 204.27 & 226.78 & 226.78 & 282.10 & 282.10 & 311.71 & 311.71 \\
\hline & $\operatorname{Ref}[11]$ & 203.29 & 203.29 & 227.17 & 227.17 & 282.35 & 282.35 & 311.15 & 311.15 \\
\hline & Present & 204.30 & 204.30 & 226.88 & 226.88 & 282.42 & 282.42 & 311.71 & 311.71 \\
\hline \multicolumn{10}{|c|}{$\mathrm{FGM}_{\mathrm{II}(a=0 / b=-0.5 / c=2 / p)}$} \\
\hline \multirow{5}{*}{0.6} & Ref [1] & 208.49 & 208.49 & 229.65 & 229.65 & 284.17 & 284.17 & 321.18 & 321.18 \\
\hline & Ref [29] & 208.54 & 208.54 & 229.70 & 229.70 & 284.25 & 284.25 & 321.30 & 321.30 \\
\hline & $\operatorname{Ref}[11]$ & 208.74 & 208.74 & 229.61 & 229.61 & 284.21 & 284.21 & 321.33 & 321.33 \\
\hline & Present & 208.57 & 208.57 & 229.80 & 229.80 & 284.58 & 284.58 & 321.30 & 321.30 \\
\hline & $\operatorname{Ref}[1]$ & 202.87 & 202.87 & 221.78 & 221.78 & 273.02 & 273.02 & 315.18 & 315.18 \\
\hline \multirow{3}{*}{5} & $\operatorname{Ref}[29]$ & 203.30 & 203.30 & 222.16 & 222.16 & 273.47 & 273.47 & 315.81 & 315.81 \\
\hline & $\operatorname{Ref}[11]$ & 204.15 & 204.15 & 221.67 & 221.67 & 273.14 & 273.14 & 315.72 & 315.72 \\
\hline & Present & 203.34 & 203.34 & 222.26 & 222.26 & 273.80 & 273.80 & 315.81 & 315.81 \\
\hline \multirow{4}{*}{20} & Ref [1] & 202.60 & 202.60 & 224.87 & 224.87 & 279.65 & 279.66 & 310.83 & 310.83 \\
\hline & Ref [29] & 203.09 & 203.09 & 225.30 & 225.30 & 280.17 & 280.17 & 311.53 & 311.53 \\
\hline & $\operatorname{Ref}[11]$ & 203.98 & 203.98 & 224.73 & 224.73 & 279.80 & 279.80 & 311.39 & 311.39 \\
\hline & Present & 203.12 & 203.12 & 225.40 & 225.40 & 280.49 & 280.49 & 311.53 & 311.53 \\
\hline
\end{tabular}

be served as benchmark data for the future numerical methods, it does not meet authors' ambition. The goal of this paper is to provide some useful research works for the designer or engineer to avoid the unpleasant, inefficient, and structurally damaging resonant by given a unified computational model of the FG cylindrical, conical, and spherical panels and shells of revolution. So, in the this subsection, the parameter studies including the boundary restraint rigidity $\Gamma$, thickness $h$, circumferential angle $\phi$, power-law exponent $p$, and material parameters $a, b$, and $c$ will be conducted.

Figure 9 shows the effects of the all kinds of boundary restraint parameters on the frequency parameters of FG panels and shells with elastic boundary conditions. Five nondimensional boundary restraint parameters $\Gamma_{b}$ $(b=u, v, w, \alpha$, and $\beta)$ are defined as ratios of corresponding spring stiffness to the reference bending stiffness $D$; that is, $\Gamma_{u}=k_{u} / D, \Gamma_{v}=k_{v} / D, \Gamma_{w}=k_{w} / D, \Gamma_{\alpha}=K_{\alpha} / D$, and $\Gamma_{\beta}=K_{\beta} / D$. The geometry properties of the panels and shells studied are the same as Tables $3-8$. The panels and shells are free at edges $\beta=$ constant, clamped at $\alpha=0$ while the $\alpha=L_{\alpha}$ is elastically restrained by all the five groups of boundary springs, of which only one group is with the variable stiffness and the others are assumed to be zeros. According to Figure 9, we can see that the change of the boundary elastic restraint parameter $\Gamma$ has little effect on the frequency parameter $\Omega$ when it is smaller than $10^{-2} \mathrm{D}$. However, when it is increased in a certain range, the frequency parameters increase rapidly as the elastic parameters increase, approach their utmost, and remain unchanged when $\Gamma$ approaches infinity. It is noted that the certain range with respect to different kinds of elastic restraint parameters is different. Figures 10 and 11 show the influence of the fundamental frequency parameters versus thickness $h$ for the FG panels and shells with different shear correction factors, respectively. It is obvious that the frequency parameters of the FG panels and shells with respect to the classical and elastic boundary condition increase when increasing the thicknesses. In addition, the figures also shows that the shear correction factor $\kappa$ have a significant impact on the vibration behavior of FG panels and shells and a larger shear correction factor will result in higher frequency parameters. Next, Figure 12 presents the first four frequency parameters for the FG panels versus the circumference angles 
TABLE 7: Comparisons of frequencies $(\mathrm{Hz})$ of the functionally graded spherical panels with different power-law index $p$.

\begin{tabular}{|c|c|c|c|c|c|c|c|c|c|c|}
\hline \multirow{2}{*}{$p$} & \multirow{2}{*}{ Method } & \multicolumn{9}{|c|}{ Mode number } \\
\hline & & 1 & 2 & 3 & 4 & 5 & 6 & 7 & 8 & 9 \\
\hline \multicolumn{11}{|c|}{$\mathrm{FGM}_{\mathrm{I}(a=1 / b=0 / c / p)}$} \\
\hline \multirow{4}{*}{0.6} & $\operatorname{Ref}[2]$ & 71.12 & 94.44 & 147.32 & 173.88 & 237.31 & 315.92 & 407.09 & 412.60 & 442.60 \\
\hline & $\operatorname{Ref}[10]$ & 71.01 & 94.37 & 147.25 & 173.87 & 237.25 & 315.55 & 406.65 & 412.62 & 441.76 \\
\hline & Present & 71.09 & 94.36 & 147.37 & 174.05 & 237.21 & 315.66 & 406.80 & 412.48 & 441.77 \\
\hline & $\operatorname{Ref}[2]$ & 70.78 & 94.02 & 146.63 & 173.14 & 236.17 & 314.53 & 405.19 & 410.79 & 440.35 \\
\hline \multirow[t]{3}{*}{1} & $\operatorname{Ref}[10]$ & 70.63 & 93.89 & 146.47 & 173.02 & 235.96 & 313.96 & 404.50 & 410.55 & 439.17 \\
\hline & Present & 70.71 & 93.88 & 146.59 & 173.21 & 235.91 & 314.09 & 404.67 & 410.40 & 439.17 \\
\hline & Ref [2] & 71.68 & 96.03 & 148.75 & 177.58 & 237.24 & 321.15 & 409.51 & 420.41 & 435.62 \\
\hline \multirow[t]{3}{*}{5} & $\operatorname{Ref}[10]$ & 71.54 & 95.89 & 148.59 & 177.46 & 237.03 & 320.58 & 408.82 & 420.09 & 434.56 \\
\hline & Present & 71.62 & 95.88 & 148.69 & 177.67 & 237.00 & 320.69 & 408.99 & 420.00 & 434.54 \\
\hline & $\operatorname{Ref}[2]$ & 70.59 & 94.55 & 146.50 & 174.82 & 233.56 & 316.21 & 403.23 & 413.98 & 428.88 \\
\hline \multirow[t]{3}{*}{20} & $\operatorname{Ref}[10]$ & 70.57 & 94.57 & 146.59 & 175.00 & 233.75 & 316.16 & 403.21 & 414.32 & 428.53 \\
\hline & Present & 70.64 & 94.56 & 146.69 & 175.20 & 233.71 & 316.28 & 403.37 & 414.24 & 428.51 \\
\hline & $\operatorname{Ref}[2]$ & 69.54 & 92.95 & 144.28 & 171.59 & 230.57 & 310.83 & 397.57 & 406.64 & 425.16 \\
\hline \multirow[t]{2}{*}{50} & $\operatorname{Ref}[10]$ & 69.57 & 93.01 & 144.45 & 171.87 & 230.90 & 310.98 & 397.80 & 407.27 & 425.06 \\
\hline & Present & 69.64 & 92.98 & 144.56 & 172.07 & 230.86 & 311.10 & 397.96 & 407.60 & 425.06 \\
\hline \multicolumn{11}{|c|}{$\mathrm{FGM}_{\mathrm{II}(a=1 / b=0 / c / p)}$} \\
\hline \multirow{4}{*}{0.6} & Ref [2] & 70.59 & 93.62 & 146.22 & 172.31 & 235.55 & 313.20 & 403.88 & 409.05 & 439.88 \\
\hline & $\operatorname{Ref}[10]$ & 70.81 & 93.98 & 146.83 & 173.10 & 236.58 & 314.28 & 405.31 & 410.95 & 441.00 \\
\hline & Present & 70.89 & 93.97 & 146.96 & 173.29 & 236.55 & 314.41 & 405.48 & 410.80 & 441.02 \\
\hline & Ref [2] & 70.12 & 92.99 & 145.26 & 171.15 & 233.94 & 311.11 & 401.15 & 406.32 & 436.81 \\
\hline \multirow[t]{3}{*}{1} & $\operatorname{Ref}[10]$ & 70.39 & 93.41 & 145.95 & 172.05 & 235.11 & 312.37 & 402.82 & 408.46 & 438.20 \\
\hline & Present & 70.48 & 93.40 & 146.08 & 172.21 & 235.08 & 312.48 & 402.97 & 408.30 & 438.21 \\
\hline & $\operatorname{Ref}[2]$ & 71.04 & 95.02 & 147.45 & 175.58 & 235.02 & 317.81 & 405.72 & 416.02 & 432.13 \\
\hline \multirow[t]{3}{*}{5} & $\operatorname{Ref}[10]$ & 71.30 & 95.41 & 148.12 & 176.44 & 236.12 & 318.99 & 407.26 & 417.98 & 433.45 \\
\hline & Present & 71.38 & 95.45 & 148.23 & 176.64 & 236.10 & 319.11 & 407.43 & 418.05 & 433.45 \\
\hline & Ref [2] & 70.33 & 94.13 & 145.98 & 174.00 & 232.64 & 314.84 & 401.67 & 412.16 & 427.43 \\
\hline \multirow[t]{3}{*}{20} & $\operatorname{Ref}[10]$ & 70.47 & 94.36 & 146.40 & 174.57 & 233.35 & 315.49 & 402.55 & 413.44 & 428.05 \\
\hline & Present & 70.55 & 94.37 & 146.50 & 174.77 & 233.33 & 315.61 & 402.71 & 413.34 & 428.03 \\
\hline & Ref [2] & 69.43 & 92.74 & 144.04 & 171.22 & 230.16 & 310.22 & 396.86 & 405.84 & 424.51 \\
\hline \multirow[t]{2}{*}{50} & $\operatorname{Ref}[10]$ & 69.54 & 92.94 & 144.41 & 171.73 & 230.79 & 310.77 & 397.61 & 407.00 & 424.96 \\
\hline & Present & 69.60 & 92.91 & 144.48 & 171.87 & 230.69 & 310.81 & 397.66 & 406.77 & 424.84 \\
\hline
\end{tabular}

with the $\mathrm{CCCC}$ and $\mathrm{E}^{3} \mathrm{E}^{3} \mathrm{E}^{3} \mathrm{E}^{3}$ boundary conditions. From the figure, we can see that the frequency parameters rapidly decrease while the circumference angle changed from $5^{\circ}$ to $75^{\circ}$, and out of this range the frequency parameters slowly minish and lastly almost remain unchanged. In addition, we also found that the variation of the frequency parameters has nothing to do with the modal order. Then, the effects of the power-law index $p$ and the material parameters $a, b$, and $c$ are studied as well. Figure 13 shows the fundamental frequency versus of the power-law index $p$ for FG panels and shells with clamped boundary conditions. The material distribution using in the study is $\operatorname{FGM}_{\mathrm{I}}(a / b / c / p)$ and $\operatorname{FGM}_{\mathrm{II}}(a / b / c / p)$ : cylindrical meridian: $\operatorname{FGM}_{\mathrm{I}(a=1 / b=0.5 / c=2 / p)}$ and $\operatorname{FGM}_{\mathrm{II}(a=1 / b=0.5 / c=2 / p)}$; conical meridian:
$\mathrm{FGM}_{\mathrm{I}(a=0 / b=-0.5 / c=2 / p)}$ and $\operatorname{FGM}_{\mathrm{II}(a=0 / b=-0.5 / c=2 / p)}$; spherical meridian: $\mathrm{FGM}_{\mathrm{I}(a=1 / b=1 / c=4 / p)}$ and $\mathrm{FGM}_{\mathrm{II}(a=1 / b=1 / c=4 / p)}$. From the figure, we can see that the geometrical construction has effect on the vibration characteristic of the FG panels and shells. For case of the cylindrical meridian, the fundamental frequency firstly rapidly decreases and then increases and finally becomes decreased versus the power-law index $p$ increased. However, for the conical and spherical meridian, the frequency parameters decrease monotonously while increasing the power-law index $p$. In addition to that, the figure also shows that the frequency parameters of the FGM structure elements are always higher than the $\mathrm{FGM}_{\mathrm{I}}$ case. In Figure 14, the influence of the combination relations between the boundary condition and power-law index $p$ on the 
TABLE 8: Comparisons of frequencies $(\mathrm{Hz})$ of the functionally graded spherical shells with different power-law index $p$.

\begin{tabular}{|c|c|c|c|c|c|c|c|c|c|c|}
\hline \multirow{2}{*}{$p$} & \multirow{2}{*}{$n$} & \multicolumn{3}{|c|}{ FF } & \multicolumn{3}{|c|}{ SS } & \multicolumn{3}{|c|}{ CC } \\
\hline & & Ref [9] & Ref [29] & Present & $\operatorname{Ref}[9]$ & Ref [29] & Present & Ref [9] & Ref [29] & Present \\
\hline \multicolumn{11}{|c|}{$\operatorname{FGM}_{\mathrm{I}(a=1 / b=0 / c / p)}$} \\
\hline \multirow{5}{*}{0.6} & 0 & 777.60 & 777.61 & 777.60 & 786.87 & 786.84 & 786.84 & 875.72 & 875.58 & 875.57 \\
\hline & 1 & 771.92 & 771.92 & 771.91 & 827.85 & 827.82 & 827.82 & 910.70 & 910.57 & 910.56 \\
\hline & 2 & 49.89 & 49.85 & 49.89 & 886.90 & 886.85 & 886.88 & 894.67 & 894.60 & 894.65 \\
\hline & 3 & 133.68 & 133.53 & 133.68 & 881.65 & 881.61 & 881.64 & 889.45 & 889.41 & 889.43 \\
\hline & 4 & 243.64 & 243.34 & 243.66 & 914.98 & 914.97 & 914.98 & 927.36 & 927.34 & 927.35 \\
\hline \multirow{5}{*}{5} & 0 & 754.66 & 754.66 & 754.66 & 773.02 & 772.99 & 772.99 & 865.53 & 865.40 & 865.40 \\
\hline & 1 & 748.83 & 748.82 & 748.83 & 812.64 & 812.61 & 812.62 & 899.65 & 899.53 & 899.54 \\
\hline & 2 & 51.61 & 51.57 & 51.61 & 862.47 & 862.42 & 862.46 & 871.46 & 871.41 & 871.44 \\
\hline & 3 & 137.94 & 137.80 & 137.95 & 858.21 & 858.18 & 858.20 & 868.66 & 868.63 & 868.65 \\
\hline & 4 & 250.90 & 250.61 & 250.92 & 895.25 & 895.23 & 895.25 & 910.61 & 910.59 & 910.60 \\
\hline \multicolumn{11}{|c|}{$\mathrm{FGM}_{\mathrm{II}(a=1 / b=0 / c / p)}$} \\
\hline \multirow{5}{*}{0.6} & 0 & 777.04 & 777.04 & 777.03 & 767.95 & 767.93 & 767.92 & 873.85 & 873.71 & 873.70 \\
\hline & 1 & 771.53 & 771.53 & 771.52 & 815.18 & 815.16 & 815.15 & 908.81 & 908.68 & 908.67 \\
\hline & 2 & 49.64 & 49.60 & 49.64 & 887.94 & 887.89 & 887.92 & 893.80 & 893.73 & 893.78 \\
\hline & 3 & 133.03 & 132.88 & 133.03 & 885.56 & 885.52 & 885.55 & 888.32 & 888.28 & 888.30 \\
\hline & 4 & 242.47 & 242.16 & 242.48 & 919.44 & 919.43 & 919.44 & 925.77 & 925.75 & 925.76 \\
\hline \multirow{5}{*}{5} & 0 & 753.73 & 753.73 & 753.73 & 749.88 & 749.86 & 749.86 & 862.76 & 862.63 & 862.63 \\
\hline & 1 & 748.18 & 748.17 & 748.17 & 796.86 & 796.84 & 796.85 & 896.89 & 896.77 & 896.77 \\
\hline & 2 & 51.29 & 51.25 & 51.29 & 864.43 & 864.39 & 864.42 & 870.08 & 870.03 & 870.07 \\
\hline & 3 & 137.10 & 136.95 & 137.10 & 863.25 & 863.22 & 863.24 & 866.87 & 866.84 & 866.84 \\
\hline & 4 & 249.38 & 249.09 & 249.42 & 900.65 & 900.63 & 900.64 & 908.16 & 908.14 & 908.16 \\
\hline
\end{tabular}

TABLE 9: First six frequency parameters $\Omega$ of the functionally graded cylindrical panels with different boundary conditions.

\begin{tabular}{|c|c|c|c|c|c|c|c|c|c|}
\hline \multirow{2}{*}{ Mode number } & \multicolumn{9}{|c|}{ Boundary condition } \\
\hline & CCCC & CSCS & CFCF & SFSF & $\mathrm{CE}^{1} \mathrm{CE}^{1}$ & $\mathrm{SE}^{3} \mathrm{SE}^{3}$ & $E^{1} E^{1} E^{1} E^{1}$ & $E^{2} E^{2} E^{2} E^{2}$ & $E^{3} E^{3} E^{3} E^{3}$ \\
\hline \multicolumn{10}{|c|}{$\operatorname{FGM}_{\mathrm{I}(a=1 / b=0 / c / p=1)}$} \\
\hline 1 & 22.789 & 20.619 & 9.583 & 7.697 & 19.726 & 22.630 & 16.566 & 20.715 & 22.590 \\
\hline 2 & 27.804 & 24.696 & 10.383 & 8.580 & 20.255 & 27.568 & 18.123 & 21.746 & 27.534 \\
\hline 3 & 36.438 & 35.286 & 18.131 & 17.363 & 31.356 & 36.345 & 18.522 & 30.208 & 36.213 \\
\hline 4 & 37.573 & 36.550 & 20.548 & 17.596 & 32.096 & 37.507 & 19.672 & 32.569 & 37.387 \\
\hline 5 & 41.149 & 39.062 & 21.822 & 17.925 & 34.202 & 40.949 & 26.910 & 34.497 & 40.931 \\
\hline 6 & 46.167 & 40.997 & 22.101 & 19.693 & 37.734 & 45.832 & 29.461 & 40.940 & 45.731 \\
\hline \multicolumn{10}{|c|}{$\mathrm{FGM}_{\mathrm{II}(a=1 / b=0 / c / p=1)}$} \\
\hline 1 & 22.647 & 21.220 & 9.573 & 7.769 & 19.572 & 22.537 & 16.544 & 20.610 & 22.479 \\
\hline 2 & 27.583 & 24.165 & 10.343 & 8.702 & 20.231 & 27.275 & 17.995 & 21.659 & 27.227 \\
\hline 3 & 36.294 & 35.914 & 18.098 & 16.773 & 31.000 & 36.261 & 18.522 & 29.997 & 36.119 \\
\hline 4 & 37.443 & 36.288 & 20.346 & 17.801 & 31.957 & 37.342 & 19.667 & 32.458 & 37.208 \\
\hline 5 & 40.975 & 40.410 & 21.806 & 18.006 & 34.164 & 40.917 & 26.922 & 34.355 & 40.901 \\
\hline 6 & 45.926 & 40.416 & 22.067 & 19.001 & 37.796 & 45.749 & 29.431 & 40.726 & 45.637 \\
\hline
\end{tabular}


TABLE 10: First six frequency parameters $\Omega$ of the functionally graded cylindrical shells with different boundary conditions.

\begin{tabular}{|c|c|c|c|c|c|c|c|c|c|}
\hline \multirow{2}{*}{ Mode number } & \multicolumn{9}{|c|}{ Boundary condition } \\
\hline & $\mathrm{CC}$ & CS & $\mathrm{CF}$ & SF & $\mathrm{CE}^{1}$ & $\mathrm{SE}^{3}$ & $E^{1} E^{1}$ & $E^{2} E^{2}$ & $E^{3} E^{3}$ \\
\hline \multicolumn{10}{|c|}{$\operatorname{FGM}_{\mathrm{I}(a=1 / b=0 / c / p=1)}$} \\
\hline 1 & 16.879 & 16.337 & 6.8893 & 6.8050 & 16.040 & 16.314 & 13.812 & 16.200 & 16.827 \\
\hline 2 & 16.879 & 16.337 & 6.8893 & 6.8050 & 16.040 & 16.314 & 14.403 & 16.200 & 16.827 \\
\hline 3 & 17.745 & 17.237 & 9.7991 & 9.7427 & 16.052 & 17.215 & 14.403 & 17.185 & 17.698 \\
\hline 4 & 17.745 & 17.237 & 9.7991 & 9.7427 & 16.052 & 17.215 & 15.248 & 17.185 & 17.698 \\
\hline 5 & 21.597 & 21.168 & 11.507 & 11.428 & 19.597 & 21.149 & 15.248 & 20.972 & 21.554 \\
\hline 6 & 21.597 & 21.168 & 11.507 & 11.428 & 19.597 & 21.149 & 15.464 & 20.972 & 21.554 \\
\hline \multicolumn{10}{|c|}{$\mathrm{FGM}_{\mathrm{II}(a=1 / b=0 / c / p=1)}$} \\
\hline 1 & 16.780 & 15.876 & 6.8620 & 6.5223 & 15.953 & 15.840 & 13.812 & 16.102 & 16.699 \\
\hline 2 & 16.780 & 15.876 & 6.8620 & 6.5223 & 15.953 & 15.840 & 14.374 & 16.102 & 16.699 \\
\hline 3 & 17.714 & 16.904 & 9.6811 & 9.5146 & 16.011 & 16.871 & 14.374 & 17.155 & 17.642 \\
\hline 4 & 17.714 & 16.904 & 9.6811 & 9.5146 & 16.011 & 16.871 & 15.147 & 17.155 & 17.642 \\
\hline 5 & 21.381 & 20.683 & 11.508 & 11.177 & 19.594 & 20.656 & 15.147 & 20.754 & 21.315 \\
\hline 6 & 21.381 & 20.683 & 11.508 & 11.177 & 19.594 & 20.656 & 15.463 & 20.754 & 21.315 \\
\hline
\end{tabular}

TABLE 11: First six frequency parameters $\Omega$ of the functionally graded conical panels with different boundary conditions.

\begin{tabular}{|c|c|c|c|c|c|c|c|c|c|}
\hline \multirow{2}{*}{ Mode number } & \multicolumn{9}{|c|}{ Boundary condition } \\
\hline & CCCC & CSCS & $\mathrm{CFCF}$ & SFSF & $\mathrm{CE}^{1} \mathrm{CE}^{1}$ & $\mathrm{SE}^{3} \mathrm{SE}^{3}$ & $E^{1} E^{1} E^{1} E^{1}$ & $E^{2} E^{2} E^{2} E^{2}$ & $E^{3} E^{3} E^{3} E^{3}$ \\
\hline \multicolumn{10}{|c|}{$\mathrm{FGM}_{\mathrm{I}(a=1 / b=0.5 / c=2 / p)}$} \\
\hline 1 & 24.598 & 22.827 & 10.062 & 8.130 & 21.649 & 22.861 & 18.335 & 23.804 & 24.476 \\
\hline 2 & 27.546 & 25.319 & 11.116 & 9.246 & 22.310 & 25.382 & 19.905 & 24.825 & 27.392 \\
\hline 3 & 35.766 & 34.653 & 19.734 & 17.732 & 30.047 & 32.676 & 25.501 & 34.596 & 35.612 \\
\hline 4 & 39.938 & 36.473 & 21.090 & 18.147 & 35.305 & 36.417 & 26.760 & 35.304 & 39.668 \\
\hline 5 & 41.194 & 38.498 & 23.559 & 20.537 & 36.797 & 38.274 & 29.303 & 37.316 & 41.058 \\
\hline 6 & 42.731 & 40.899 & 24.102 & 20.650 & 41.696 & 39.241 & 32.699 & 38.101 & 42.355 \\
\hline \multicolumn{10}{|c|}{$\mathrm{FGM}_{\mathrm{II}(a=1 / b=0.5 / c=2 / p)}$} \\
\hline 1 & 24.562 & 23.102 & 10.060 & 8.2037 & 21.610 & 22.706 & 18.325 & 23.773 & 24.442 \\
\hline 2 & 27.506 & 25.226 & 11.104 & 9.3506 & 22.300 & 25.232 & 19.866 & 24.793 & 27.338 \\
\hline 3 & 35.737 & 35.024 & 19.725 & 17.659 & 29.983 & 32.942 & 25.502 & 34.572 & 35.608 \\
\hline 4 & 39.882 & 36.565 & 21.053 & 17.835 & 35.291 & 36.496 & 26.767 & 35.263 & 39.626 \\
\hline 5 & 41.166 & 38.973 & 23.545 & 20.502 & 36.737 & 38.180 & 29.240 & 37.282 & 41.021 \\
\hline 6 & 42.646 & 40.733 & 24.085 & 20.688 & 41.606 & 39.057 & 32.684 & 38.059 & 42.281 \\
\hline
\end{tabular}

vibration behavior for the FG panes and shells is reported. And to simplify the study, the research selects cylindrical panels as the example. From the figure, we can know that the variation trend of the fundamental frequency of the FG panes and shells with CFCF, $\mathrm{E}^{3} \mathrm{E}^{3} \mathrm{E}^{3} \mathrm{E}^{3}$, and SSSS is firstly rapidly decreased and then increased and finally becomes decreased versus the power-law index $p$ increase. However, the frequency parameters increase rapidly as the power-law index $p$ increases, approaches their utmost, and remains unchanged when $p$ is more than a threshold. Thus, we can get that the boundary condition plays an indispensable role in the vibration behavior of the FG panes and shells. Finally, the influence of the material parameters $a, b$, and $c$ will be exhibited in the next example. Figure 15 depicts the variations of the first frequencies of the clamped supported $\mathrm{FGM}_{\mathrm{I}}$ panels and shells with different material types: Type 1: $a=1$ and $b=c=0$; Type 2: $a=1, b=0.5$, and $c=2$; Type 3: $a=0, b=-0.5$, and $c=2$; Type $4: a=1, b=1$, and $c=4$. From the figure, it is obvious that the material parameters $a, b$, and $c$ have a significant influence on the fundamental frequencies for the FG panels and shells.

\section{Conclusions}

In this paper, a unified computational model is presented for the vibration analysis of the four-parameter functionally 
TABLE 12: First six frequency parameters $\Omega$ of the functionally graded conical shells with different boundary conditions.

\begin{tabular}{|c|c|c|c|c|c|c|c|c|c|}
\hline \multirow{2}{*}{ Mode number } & \multicolumn{9}{|c|}{ Boundary condition } \\
\hline & $\mathrm{CC}$ & CS & $\mathrm{CF}$ & SF & $\mathrm{CE}^{1}$ & $\mathrm{SE}^{3}$ & $E^{1} E^{1}$ & $E^{2} E^{2}$ & $E^{3} E^{3}$ \\
\hline \multicolumn{10}{|c|}{$\mathrm{FGM}_{\mathrm{I}(a=1 / b=0.5 / c=2 / p)}$} \\
\hline 1 & 18.648 & 17.326 & 3.1641 & 3.0306 & 15.986 & 18.457 & 15.709 & 18.184 & 18.591 \\
\hline 2 & 18.648 & 17.328 & 3.1641 & 3.0306 & 15.986 & 18.460 & 15.709 & 18.186 & 18.594 \\
\hline 3 & 19.583 & 18.595 & 4.6352 & 4.4011 & 16.670 & 19.183 & 16.583 & 19.147 & 19.526 \\
\hline 4 & 19.583 & 18.595 & 4.6352 & 4.4011 & 16.670 & 19.183 & 16.583 & 19.148 & 19.526 \\
\hline 5 & 22.612 & 20.687 & 5.3016 & 5.2968 & 19.945 & 22.529 & 18.536 & 21.878 & 22.540 \\
\hline 6 & 22.612 & 20.707 & 5.3016 & 5.2968 & 19.945 & 22.549 & 18.536 & 21.898 & 22.560 \\
\hline \multicolumn{10}{|c|}{$\mathrm{FGM}_{\mathrm{II}(a=1 / b=0.5 / c=2 / p)}$} \\
\hline 1 & 18.618 & 17.080 & 3.1594 & 2.9584 & 15.977 & 18.386 & 15.699 & 18.154 & 18.552 \\
\hline 2 & 18.618 & 17.080 & 3.1594 & 2.9584 & 15.977 & 18.386 & 15.699 & 18.154 & 18.554 \\
\hline 3 & 19.572 & 18.443 & 4.6350 & 4.2895 & 16.640 & 19.099 & 16.553 & 19.137 & 19.507 \\
\hline 4 & 19.572 & 18.443 & 4.6350 & 4.2895 & 16.640 & 19.099 & 16.553 & 19.137 & 19.507 \\
\hline 5 & 22.564 & 20.403 & 5.2888 & 5.2801 & 19.943 & 22.468 & 18.535 & 21.834 & 22.483 \\
\hline 6 & 22.564 & 20.403 & 5.2888 & 5.2801 & 19.943 & 22.468 & 18.535 & 21.834 & 22.503 \\
\hline
\end{tabular}

TABLE 13: First six frequency parameters $\Omega$ of the functionally graded spherical panels with different boundary conditions.

\begin{tabular}{|c|c|c|c|c|c|c|c|c|c|}
\hline \multirow{2}{*}{ Mode number } & \multicolumn{9}{|c|}{ Boundary condition } \\
\hline & CCCC & CSCS & CFCF & SFSF & $\mathrm{CE}^{1} \mathrm{CE}^{1}$ & $\mathrm{SE}^{3} \mathrm{SE}^{3}$ & $E^{1} E^{1} E^{1} E^{1}$ & $E^{2} E^{2} E^{2} E^{2}$ & $E^{3} E^{3} E^{3} E^{3}$ \\
\hline \multicolumn{10}{|c|}{$\mathrm{FGM}_{\mathrm{I}(a=1 / b=1 / c=4 / p)}$} \\
\hline 1 & 13.652 & 13.168 & 10.001 & 9.2907 & 12.374 & 12.924 & 5.215 & 11.613 & 13.567 \\
\hline 2 & 14.297 & 14.090 & 10.057 & 9.3816 & 12.916 & 13.716 & 5.400 & 13.010 & 14.243 \\
\hline 3 & 17.060 & 16.157 & 12.518 & 10.917 & 15.840 & 14.095 & 7.290 & 13.117 & 16.827 \\
\hline 4 & 17.423 & 17.179 & 13.685 & 11.347 & 16.595 & 16.611 & 7.398 & 13.345 & 17.256 \\
\hline 5 & 20.023 & 18.796 & 14.830 & 11.965 & 16.892 & 17.493 & 10.310 & 14.562 & 19.753 \\
\hline 6 & 20.561 & 19.503 & 14.882 & 12.895 & 19.200 & 19.648 & 13.078 & 15.491 & 20.344 \\
\hline \multicolumn{10}{|c|}{$\mathrm{FGM}_{\mathrm{II}(a=1 / b=1 / c=4 / p)}$} \\
\hline 1 & 13.639 & 13.182 & 9.9925 & 9.4012 & 12.367 & 12.968 & 5.216 & 11.607 & 13.560 \\
\hline 2 & 14.286 & 14.054 & 10.051 & 9.4953 & 12.902 & 13.764 & 5.401 & 13.001 & 14.234 \\
\hline 3 & 17.037 & 16.214 & 12.508 & 10.878 & 15.817 & 14.029 & 7.282 & 13.110 & 16.801 \\
\hline 4 & 17.401 & 17.167 & 13.668 & 11.330 & 16.599 & 16.660 & 7.399 & 14.553 & 17.243 \\
\hline 5 & 19.993 & 18.755 & 14.805 & 11.985 & 16.867 & 17.486 & 10.295 & 15.478 & 19.726 \\
\hline 6 & 20.531 & 19.495 & 14.860 & 12.923 & 19.170 & 19.644 & 13.073 & 15.824 & 20.314 \\
\hline
\end{tabular}

graded moderately thick cylindrical, conical, spherical panels and shells of revolution with general boundary condition based on first-order shear deformation theory. Applying the modified Fourier-Ritz approach, the admissible functions of the structure elements are expanded into the improved Fourier series which consist of the two-dimensional (2D) Fourier cosine series and auxiliary functions to eliminate all the relevant discontinuities of the displacements and their derivatives at the edges regardless of boundary conditions and then solve the natural frequencies by means of the Ritz method. In order to obtain the unified computational model of the functionally graded cylindrical, conical, spherical panels and shells of revolution, as one merit of this paper, the coupling spring technology is introduced to ensure the kinematic and physical compatibility at the common meridian, if a complete shell of revolution needs considering. The good convergence, excellent accuracy, and reliability of the present unified computational model are checked and validated by the comparison with the results presented by other contributors. Through the parameter studies, the vibration behavior of the FG cylindrical, conical, spherical panels and shells is not only related to the boundary conditions, but also related to their self-attribute. Comparing with the existing works, the present unified computational model contains the following highlights:

(1) The proposed unified computational model is appropriate for problems which is the four-parameter functionally graded moderately thick cylindrical, conical, spherical panels and shells of revolution with general boundary condition. 
TABLE 14: First six frequency parameters $\Omega$ of the functionally graded spherical shells with different boundary conditions.

\begin{tabular}{|c|c|c|c|c|c|c|c|c|c|}
\hline \multirow{2}{*}{ Mode number } & \multicolumn{9}{|c|}{ Boundary condition } \\
\hline & $\mathrm{CC}$ & CS & $\mathrm{CF}$ & SF & $\mathrm{CE}^{1}$ & $\mathrm{SE}^{3}$ & $E^{1} E^{1}$ & $E^{2} E^{2}$ & $E^{3} E^{3}$ \\
\hline \multicolumn{10}{|c|}{$\mathrm{FGM}_{\mathrm{I}(a=1 / b=1 / c=4 / p)}$} \\
\hline 1 & 12.293 & 11.727 & 1.7433 & 1.4432 & 6.5531 & 11.970 & 5.6226 & 10.036 & 12.242 \\
\hline 2 & 12.293 & 11.727 & 1.7433 & 1.4432 & 6.5531 & 11.970 & 6.3197 & 10.838 & 12.242 \\
\hline 3 & 12.552 & 11.918 & 2.7986 & 2.2795 & 7.2370 & 12.233 & 6.3199 & 10.838 & 12.488 \\
\hline 4 & 12.552 & 11.918 & 2.7986 & 2.2795 & 8.2529 & 12.233 & 7.6911 & 12.060 & 12.488 \\
\hline 5 & 13.316 & 12.855 & 3.0960 & 3.0509 & 8.2529 & 12.992 & 7.6912 & 12.060 & 13.286 \\
\hline 6 & 13.316 & 12.855 & 3.0960 & 3.0510 & 10.393 & 12.992 & 8.6638 & 12.141 & 13.286 \\
\hline \multicolumn{10}{|c|}{$\operatorname{FGM}_{\mathrm{II}(a=1 / b=1 / c=4 / p)}$} \\
\hline 1 & 12.284 & 11.760 & 1.7406 & 1.4246 & 6.5481 & 12.002 & 5.6204 & 10.032 & 12.238 \\
\hline 2 & 12.284 & 11.760 & 1.7406 & 1.4246 & 6.5481 & 12.002 & 6.3145 & 10.833 & 12.238 \\
\hline 3 & 12.539 & 11.946 & 2.7974 & 2.2396 & 7.2331 & 12.251 & 6.3145 & 10.833 & 12.481 \\
\hline 4 & 12.539 & 11.946 & 2.7974 & 2.2396 & 8.2452 & 12.251 & 7.6835 & 12.055 & 12.481 \\
\hline 5 & 13.310 & 12.899 & 3.0896 & 3.0439 & 8.2452 & 13.032 & 7.6835 & 12.055 & 13.285 \\
\hline 6 & 13.310 & 12.899 & 3.0896 & 3.0439 & 10.381 & 13.032 & 8.6633 & 12.132 & 13.285 \\
\hline
\end{tabular}

(2) The change of the boundary conditions can be easily achieved by just varying the stiffness of the boundary restraining springs along all the edges of plates without making any changes in the solution procedure.

\section{Appendix}

\section{Detailed Expressions of the Matrices} $M, K$, and $H$

$$
\begin{aligned}
\mathbf{K} & =\left[\begin{array}{lllll}
\mathbf{K}_{u u} & \mathbf{K}_{u v} & \mathbf{K}_{u w} & \mathbf{K}_{u \alpha} & \mathbf{K}_{u \beta} \\
\mathbf{K}_{u v}^{T} & \mathbf{K}_{v v} & \mathbf{K}_{v w} & \mathbf{K}_{v \alpha} & \mathbf{K}_{v \beta} \\
\mathbf{K}_{u w}^{T} & \mathbf{K}_{v w}^{T} & \mathbf{K}_{w w} & \mathbf{K}_{w \alpha} & \mathbf{K}_{w \beta} \\
\mathbf{K}_{u \alpha}^{T} & \mathbf{K}_{v \alpha}^{T} & \mathbf{K}_{w \alpha}^{T} & \mathbf{K}_{\alpha \alpha} & \mathbf{K}_{\alpha \beta} \\
\mathbf{K}_{u \beta}^{T} & \mathbf{K}_{v \beta}^{T} & \mathbf{K}_{w \beta}^{T} & \mathbf{K}_{\alpha \beta}^{T} & \mathbf{K}_{\beta \beta}
\end{array}\right], \\
\mathbf{M} & =\left[\begin{array}{ccccc}
\mathbf{M}_{u u} & \mathbf{0} & \mathbf{0} & \mathbf{M}_{u \alpha} & \mathbf{0} \\
\mathbf{0} & \mathbf{M}_{v v} & \mathbf{0} & \mathbf{0} & \mathbf{M}_{v \beta} \\
\mathbf{0} & \mathbf{0} & \mathbf{M}_{w w} & \mathbf{0} & \mathbf{0} \\
\mathbf{M}_{u \alpha}^{T} & \mathbf{0} & \mathbf{0} & \mathbf{M}_{\alpha \alpha} & \mathbf{0} \\
\mathbf{0} & \mathbf{M}_{v \beta}^{T} & \mathbf{0} & \mathbf{0} & \mathbf{M}_{\beta \beta}
\end{array}\right], \\
\mathbf{H} & =\left[\begin{array}{c}
\mathbf{H}_{u} \\
\mathbf{H}_{v} \\
\mathbf{H}_{w} \\
\mathbf{H}_{\alpha} \\
\mathbf{H}_{\beta}
\end{array}\right) \\
\mathbf{K}_{u u} & =\iint\left\{A_{11} \frac{1}{A^{2}} \frac{\partial \mathbf{U}^{T}}{\partial \alpha} \frac{\partial \mathbf{U}}{\partial \alpha}+A_{11}\left(\frac{1}{A B} \frac{\partial B}{\partial \alpha}\right)^{2} \mathbf{U}^{T} \mathbf{U}+\kappa A_{66}\right.
\end{aligned}
$$

$$
\begin{aligned}
& \left.+A_{12}\left(\frac{1}{A^{2} B} \frac{\partial B}{\partial \alpha} \frac{\partial \mathbf{U}^{T}}{\partial \alpha} \mathbf{U}+\frac{1}{A^{2} B} \frac{\partial B}{\partial \alpha} \frac{\partial \mathbf{U}}{\partial \alpha} \mathbf{U}^{T}\right)\right\} d S \\
& +\iint\left\{k_{\alpha 0}^{u} \mathbf{U}^{T} \mathbf{U}\right\}_{\mid \alpha=0} d S_{0}+\iint\left\{k_{\alpha 1}^{u} \mathbf{U}^{T} \mathbf{U}\right\}_{\mid \alpha=L_{\varphi}} d S_{1} \\
& +\iint\left\{k_{\beta 0}^{u} \mathbf{U}^{T} \mathbf{U}\right\}_{\mid \beta=0} d S_{2}+\iint\left\{k_{\beta 1}^{u} \mathbf{U}^{T} \mathbf{U}\right\}_{\mid \beta=L_{\beta}} d S_{3} \\
& +\iint\left\{k_{u c}^{u}\left(\mathbf{U}_{\mid \beta=0}-\mathbf{U}_{\mid \beta=2 \pi}\right)^{T}\left(\mathbf{U}_{\mid \beta=0}-\mathbf{U}_{\mid \beta=2 \pi}\right)\right\} d S_{2} \\
& \mathbf{K}_{u v}=\iint\left\{A_{11} \frac{1}{A^{2} B} \frac{\partial A}{\partial \beta}\left(\frac{\partial \mathbf{U}^{T}}{\partial \alpha} \mathbf{V}+\mathbf{V}^{T} \frac{\partial \mathbf{U}}{\partial \alpha}\right)+A_{11} \frac{1}{A B^{2}}\right. \\
& \cdot \frac{\partial B}{\partial \alpha}\left(\frac{\partial \mathbf{V}^{T}}{\partial \beta} \mathbf{U}+\mathbf{U}^{T} \frac{\partial \mathbf{V}}{\partial \beta}\right)+A_{66}\left(\frac{\partial}{\partial \alpha}\left(\frac{\mathbf{V}^{T}}{B}\right) \frac{\partial}{\partial \beta}\left(\frac{\mathbf{U}}{A}\right)\right. \\
& \left.+\frac{\partial}{\partial \alpha}\left(\frac{\mathbf{V}}{B}\right) \frac{\partial}{\partial \beta}\left(\frac{\mathbf{U}^{T}}{A}\right)\right)+A_{12} \frac{1}{A B}\left(\frac{\partial \mathbf{U}^{T}}{\partial \alpha} \frac{\partial \mathbf{V}}{\partial \beta}\right. \\
& \left.\left.+\frac{\partial \mathbf{U}}{\partial \alpha} \frac{\partial \mathbf{V}^{T}}{\partial \beta}\right)+A_{12} \frac{1}{A^{2} B^{2}} \frac{\partial A}{\partial \beta} \frac{\partial B}{\partial \alpha}\left(\mathbf{U}^{T} \mathbf{V}+\mathbf{V}^{T} \mathbf{U}\right)\right\} d S \\
& \mathbf{K}_{u w}=\iint\left\{A_{11} \frac{1}{A R_{\alpha}}\left(\frac{\partial \mathbf{U}^{T}}{\partial \alpha} \mathbf{W}+\mathbf{W}^{T} \frac{\partial \mathbf{U}}{\partial \alpha}\right)+A_{11} \frac{1}{A B}\right. \\
& \cdot \frac{\partial B}{\partial \alpha R_{\beta}}\left(\mathbf{U}^{T} \mathbf{W}+\mathbf{W}^{T} \mathbf{U}\right)+\kappa A_{66} \frac{1}{A R_{\alpha}}\left(\frac{\partial \mathbf{W}^{T}}{\partial \alpha} \mathbf{U}\right. \\
& \left.+\mathbf{U}^{T} \frac{\partial \mathbf{W}}{\partial \alpha}\right)+A_{12} \frac{1}{A R_{\beta}}\left(\frac{\partial \mathbf{U}^{T}}{\partial \alpha} \mathbf{W}+\mathbf{W}^{T} \frac{\partial \mathbf{U}}{\partial \alpha}\right)+A_{12} \frac{1}{A B} \\
& \left.\cdot \frac{\partial B}{\partial \alpha R_{\alpha}}\left(\mathbf{U}^{T} \mathbf{W}+\mathbf{W}^{T} \mathbf{U}\right)\right\} d S \\
& \mathbf{K}_{u \alpha}=\iint\left\{\kappa A_{66} \frac{1}{R_{\alpha}}\left(\mathbf{U}^{T} \boldsymbol{\Phi}+\mathbf{U} \boldsymbol{\Phi}^{T}\right)+B_{11} \frac{1}{A^{2}}\left(\frac{\partial \mathbf{U}^{T}}{\partial \alpha} \frac{\partial \boldsymbol{\Phi}}{\partial \alpha}\right.\right. \\
& \left.+\frac{\partial \mathbf{U}}{\partial \alpha} \frac{\partial \boldsymbol{\Phi}^{T}}{\partial \alpha}\right)+B_{12} \frac{1}{A^{2} B} \frac{\partial B}{\partial \alpha}\left(\frac{\partial \mathbf{U}^{T}}{\partial \alpha} \boldsymbol{\Phi}+\frac{\partial \mathbf{U}}{\partial \alpha} \boldsymbol{\Phi}^{T}\right)+B_{12}
\end{aligned}
$$


Cylindrical panels: SFSF

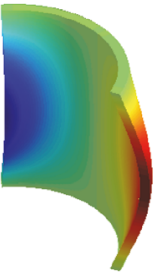

1st mode

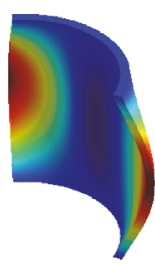

2nd mode

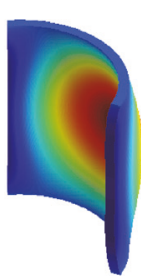

3rd mode

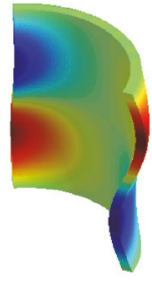

4th mode

Cylindrical panels: $\mathrm{E}^{1} \mathrm{E}^{1} \mathrm{E}^{1} \mathrm{E}^{1}$
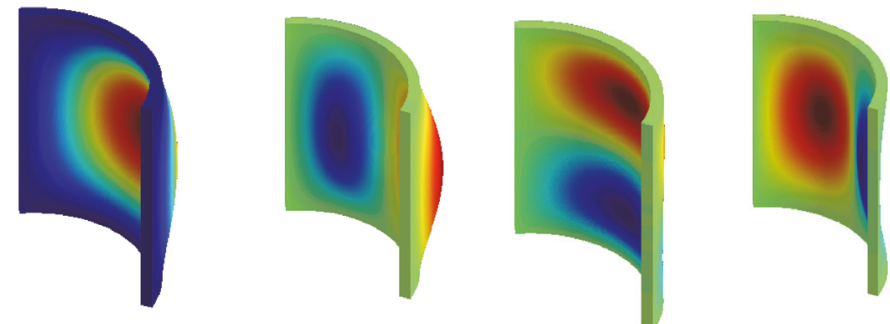

1st mode

2nd mode

3rd mode

4th mode

Cylindrical panels: $\mathrm{E}^{3} \mathrm{E}^{3} \mathrm{E}^{3} \mathrm{E}^{3}$

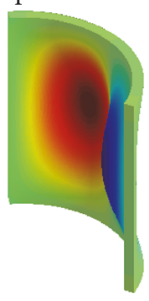

1st mode

Cylindrical shell: CF

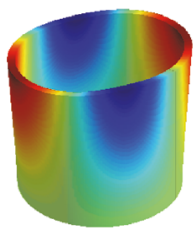

1st-2nd mode

Cylindrical shell: $\mathrm{E}^{1} \mathrm{E}^{1}$

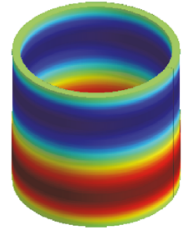

1st mode

Cylindrical shell: $\mathrm{E}^{3} \mathrm{E}^{3}$

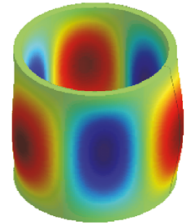

1st-2nd mode

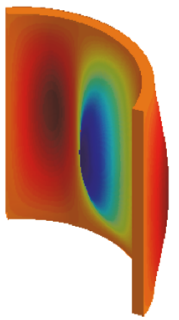

2nd mode

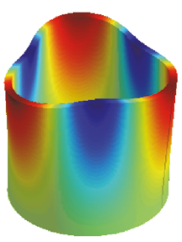

3rd-4th mode

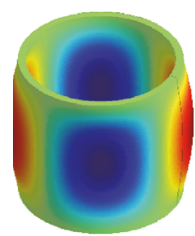

2nd-3rd mode

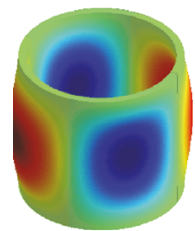

3rd-4th mode

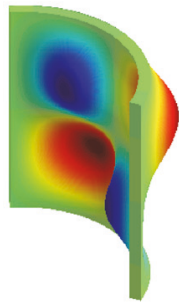

3rd mode

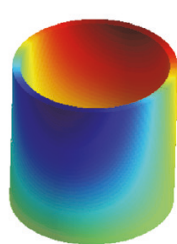

5th-6th mode

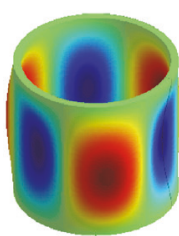

4th-5th mode

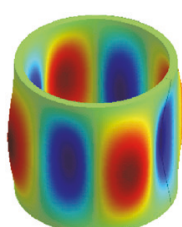

5th-6th mode

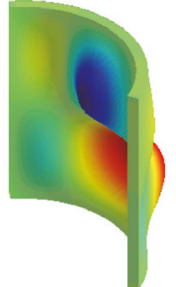

4th mode

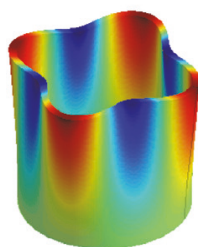

7th-8th mode

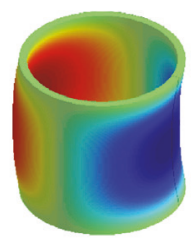

6th-7th mode

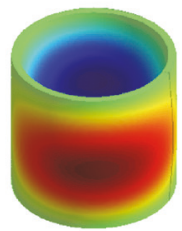

7th-8th mode

FIGURE 6: Mode shapes for cylindrical panels and shells with different boundary conditions. 
Conical panels: CFCF

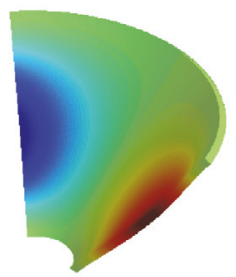

1st mode

Conical panels: $\mathrm{E}^{1} \mathrm{E}^{1} \mathrm{E}^{1} \mathrm{E}^{1}$

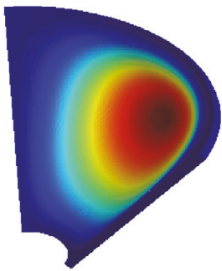

1st mode

Conical panels: $\mathrm{E}^{3} \mathrm{E}^{3} \mathrm{E}^{3} \mathrm{E}^{3}$

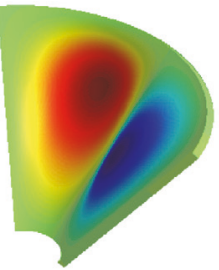

1st mode

Conical shell: $\mathrm{CF}$

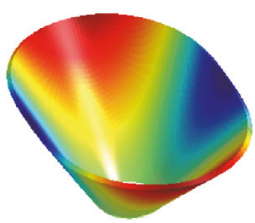

1st-2nd mode

Conical shell: $\mathrm{E}^{1} \mathrm{E}^{1}$

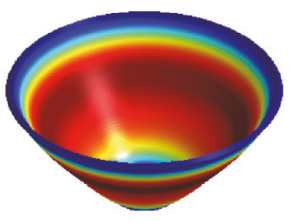

1st mode

Conical shell: $\mathrm{E}^{3} \mathrm{E}^{3}$

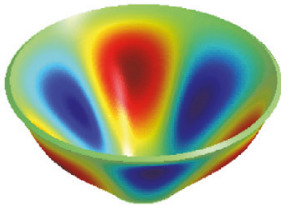

1st-2nd mode

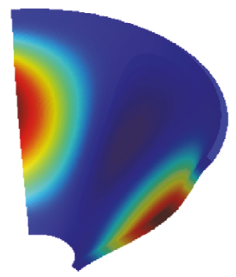

2nd mode

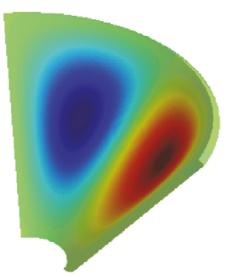

2nd mode

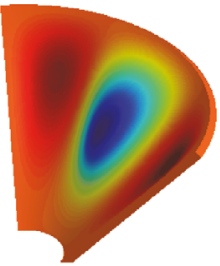

2nd mode

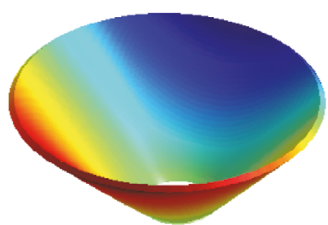

3rd-4th mode

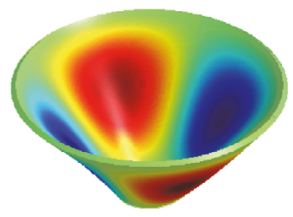

2nd-3rd mode

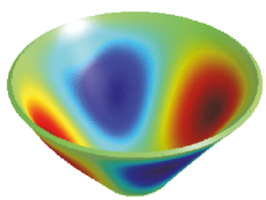

3rd-4th mode

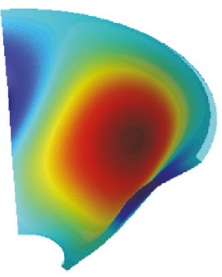

3rd mode

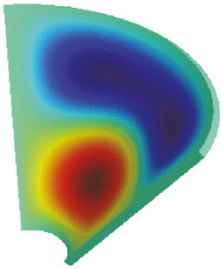

3rd mode

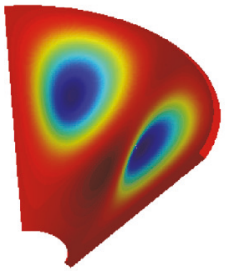

3rd mode

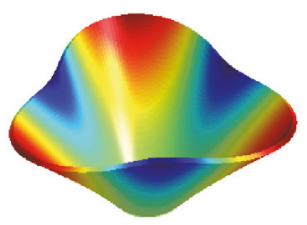

5th-6th mode

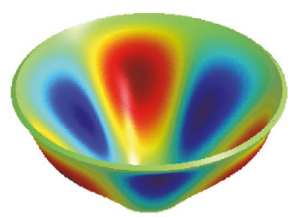

4th-5th mode

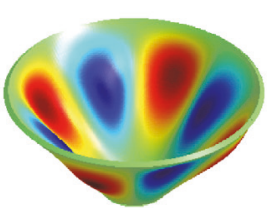

5th-6th mode

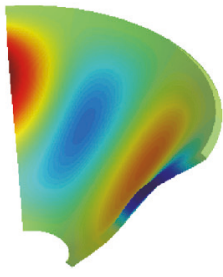

4th mode

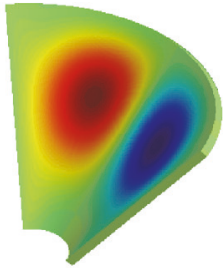

4th mode

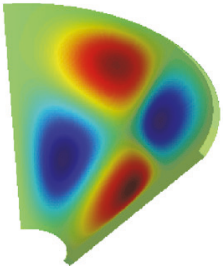

4th mode

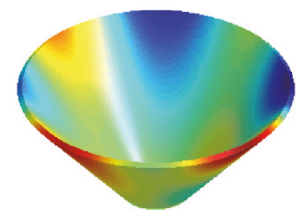

7th-8th mode

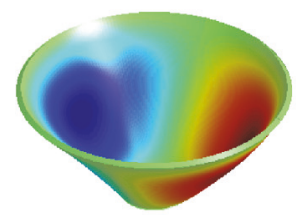

6th-7th mode

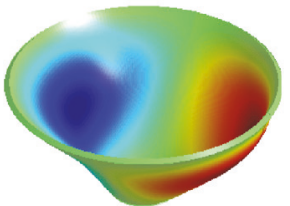

7th-8th mode

FIgURE 7: Mode shapes for conical panels and shells with different boundary conditions. 
Spherical panels: CFCF

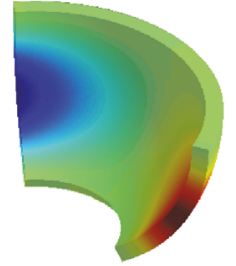

1st mode

Spherical panels: $\mathrm{E}^{1} \mathrm{E}^{1} \mathrm{E}^{1} \mathrm{E}^{1}$

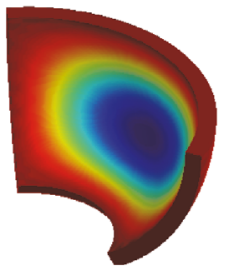

1 st mode

Spherical panels: $\mathrm{E}^{3} \mathrm{E}^{3} \mathrm{E}^{3} \mathrm{E}^{3}$

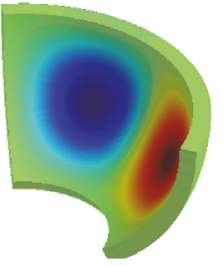

1st mode

Spherical shell: CF

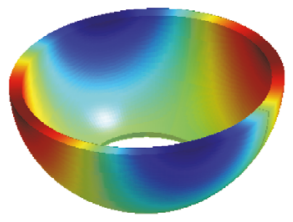

1st-2nd mode

Spherical shell: $\mathrm{E}^{1} \mathrm{E}^{1}$

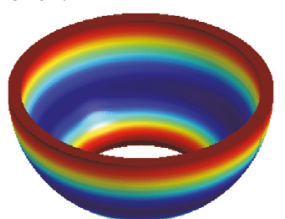

1st mode

Spherical shell: $\mathrm{E}^{3} \mathrm{E}^{3}$

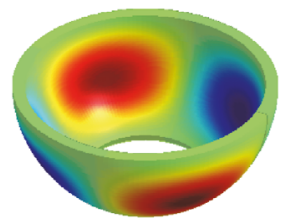

1st-2nd mode

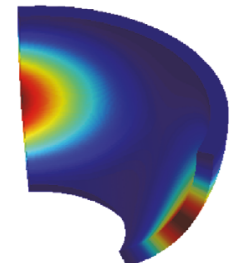

2nd mode

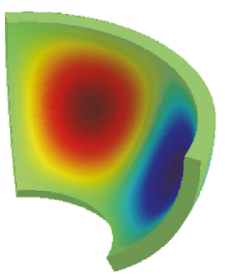

2nd mode

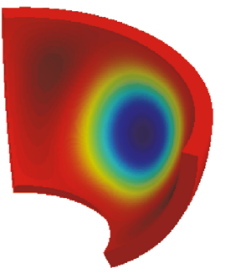

2nd mode

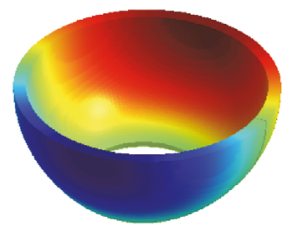

3rd-4th mode

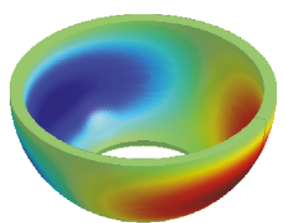

2nd-3rd mode

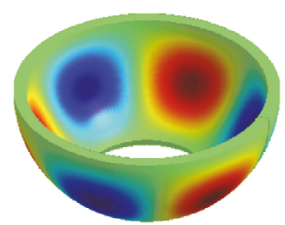

3rd-4th mode

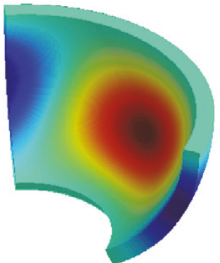

3rd mode

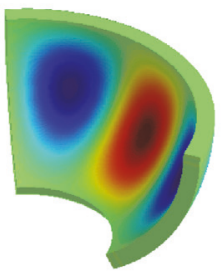

3rd mode

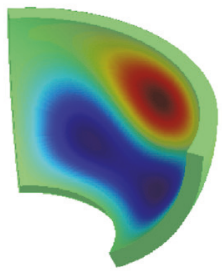

3rd mode

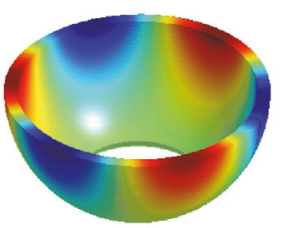

5th-6th mode

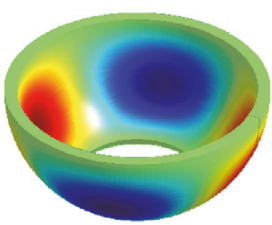

4th-5th mode

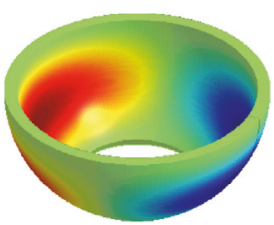

5th-6th mode

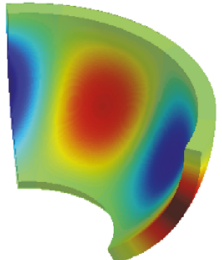

4th mode

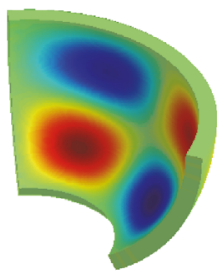

4th mode

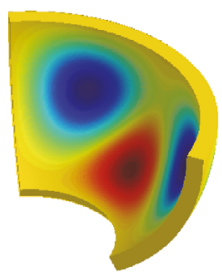

4th mode

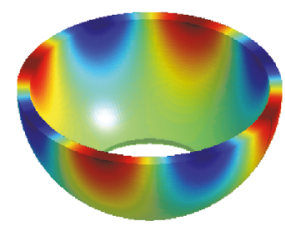

7th-8th mode

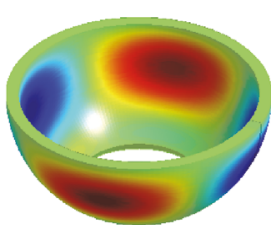

6th-7th mode

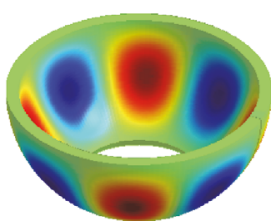

7th-8th mode

FIGURE 8: Mode shapes for spherical panels and shells with different boundary conditions. 

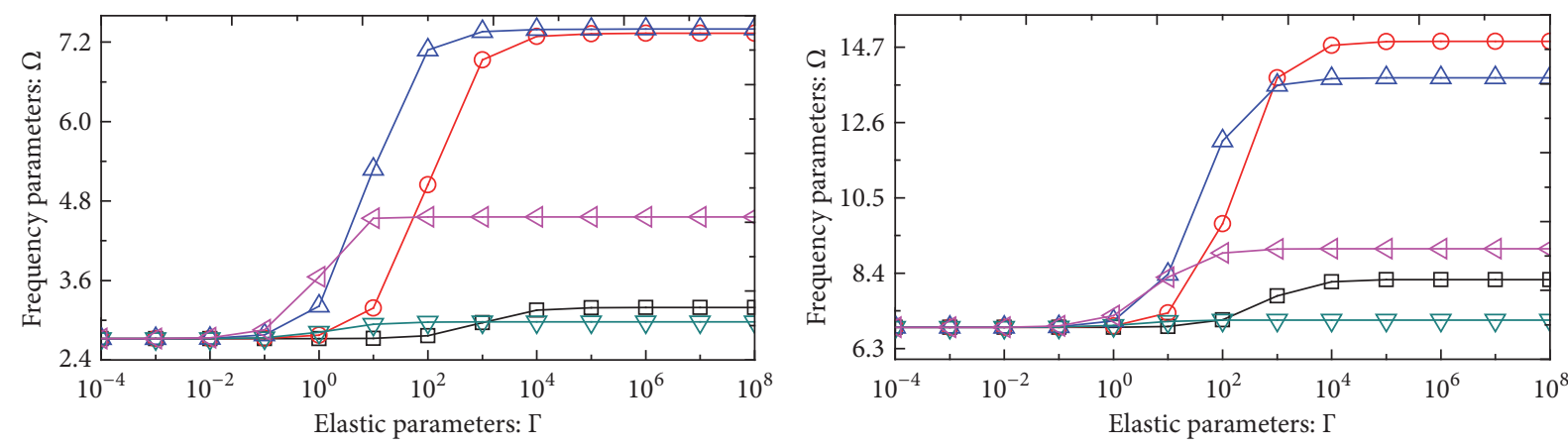

$$
\begin{aligned}
& \square-\Gamma_{u} \\
& \bigcirc-\Gamma_{v} \\
& \triangle \Gamma_{w}
\end{aligned}
$$

$$
\begin{array}{ll}
\nabla & \Gamma_{\varphi} \\
\varangle & \Gamma_{\theta}
\end{array}
$$

(a)

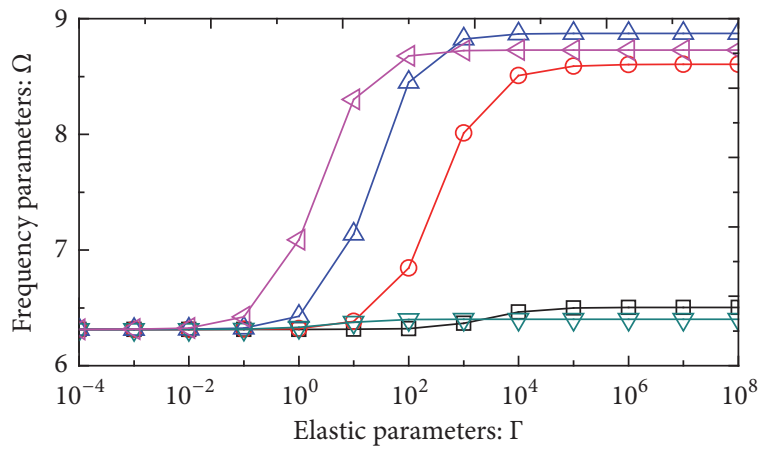

$$
\begin{array}{ll}
\square & \Gamma_{u} \\
\bigcirc & \Gamma_{v} \\
\triangle & \Gamma_{w}
\end{array}
$$$$
\begin{array}{ll}
\nabla & \Gamma_{\varphi} \\
\varangle & \Gamma_{\theta}
\end{array}
$$

(c)

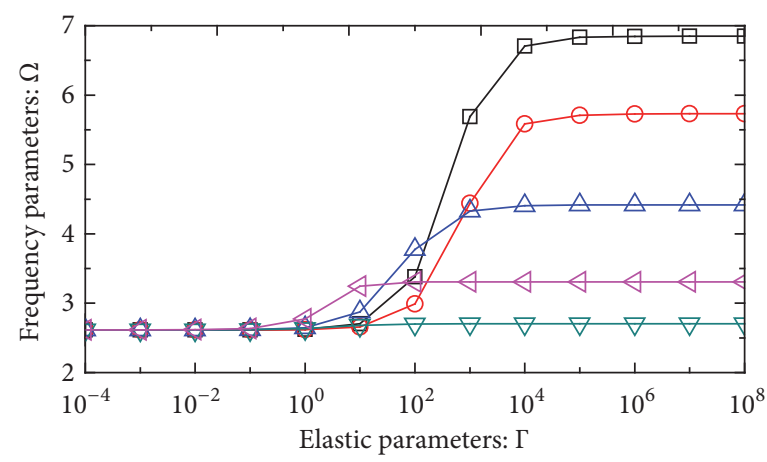

$$
\begin{array}{ll}
\square & \Gamma_{u} \\
- & \Gamma_{v} \\
\triangle & \Gamma_{w}
\end{array}
$$$$
\begin{array}{ll}
\nabla & \Gamma_{\varphi} \\
\varangle & \Gamma_{\theta}
\end{array}
$$

(e)

$$
\begin{array}{ll}
\square \Gamma_{u} & \nabla \Gamma_{\varphi} \\
\bigcirc-\Gamma_{v} & \smile \Gamma_{\theta} \\
\triangle \Gamma_{w} &
\end{array}
$$

(b)

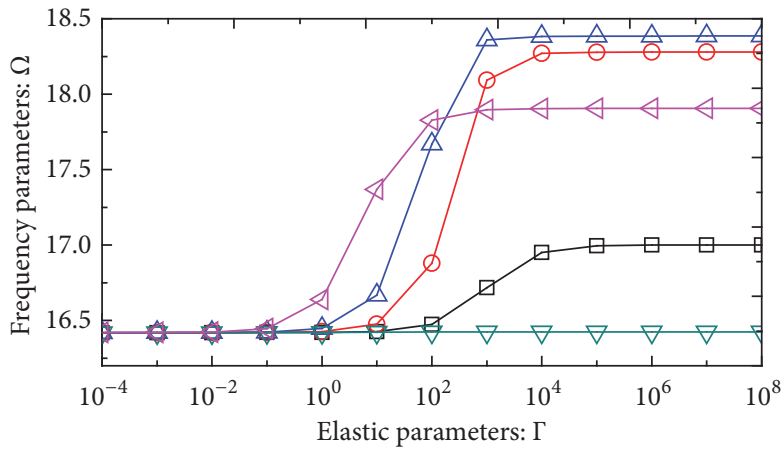

$$
\begin{aligned}
& \square \Gamma_{u} \\
& \circ \Gamma_{v} \\
& \begin{array}{ll}
\nabla & \Gamma_{\varphi} \\
\varangle & \Gamma_{\theta}
\end{array}
\end{aligned}
$$$$
\triangle \Gamma_{w}
$$

(d)

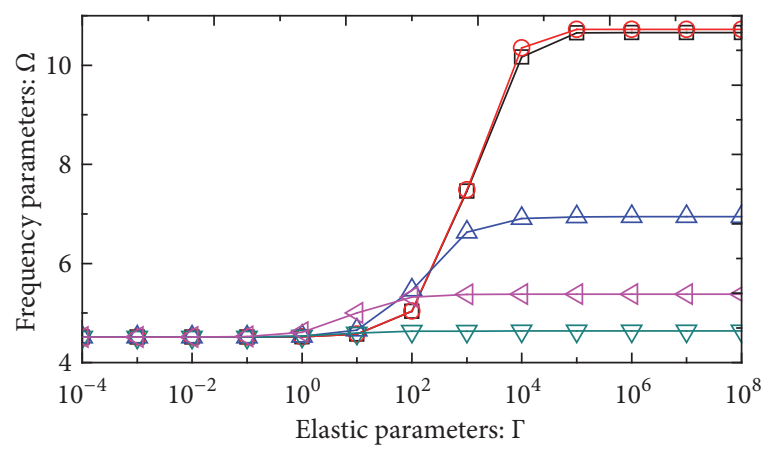

$$
\begin{array}{cc}
\square & \Gamma_{u} \\
\ominus & \Gamma_{v}
\end{array}
$$$$
\begin{array}{ll}
\nabla & \Gamma_{\varphi} \\
\varangle & \Gamma_{\theta}
\end{array}
$$

(f)

FIGURE 9: Variation of the fundamental frequency parameters $\Omega$ versus the elastic restraint parameters for FG panels and shells: (a) cylindrical panels; (b) cylindrical shells; (c) conical panels; (d) conical shells; (e) spherical panels; (f) spherical shells. 

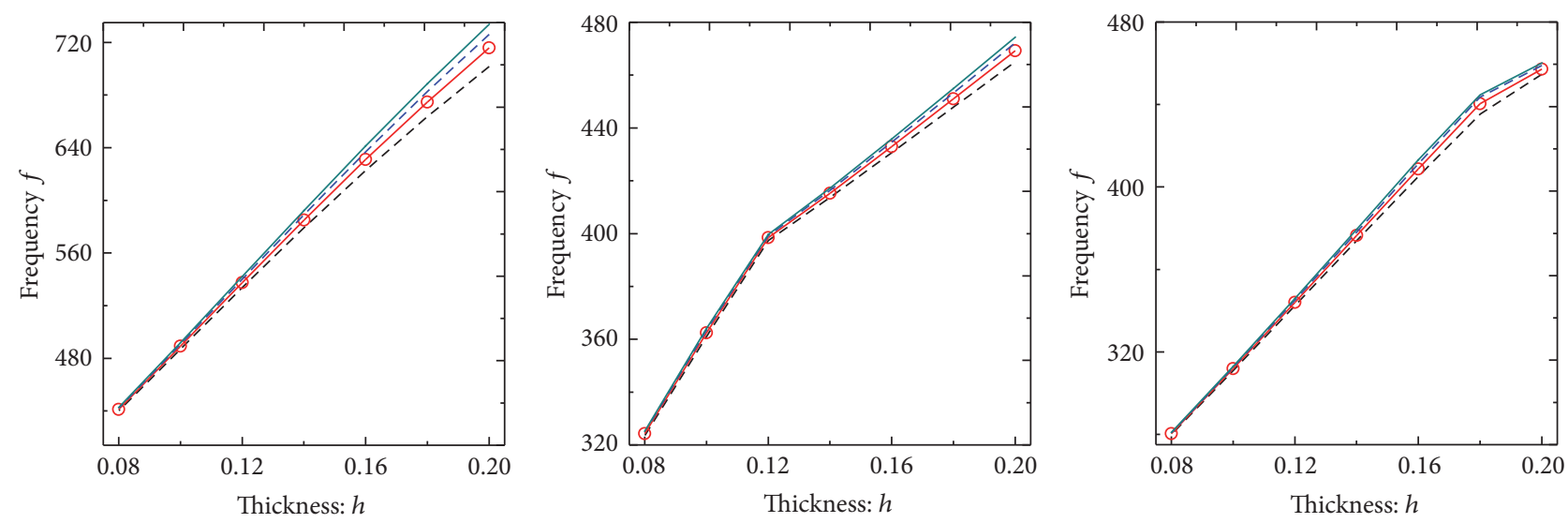

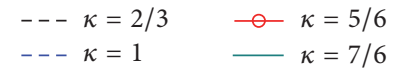

(a)

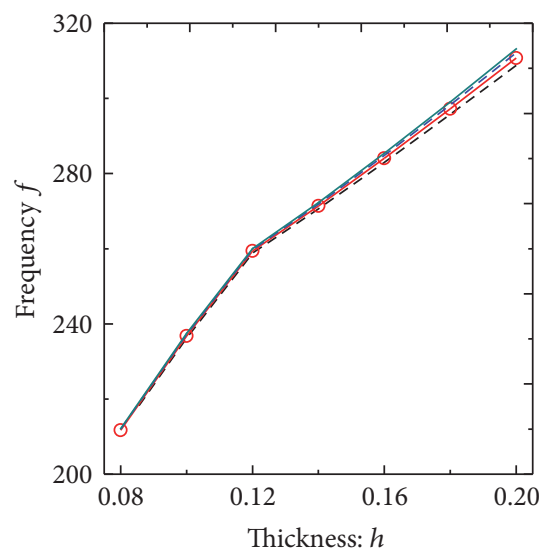

$\begin{array}{llrl}--\kappa & =2 / 3 & -\kappa & =5 / 6 \\ --\kappa & =1 & -\kappa & =7 / 6\end{array}$

(d)

\begin{abstract}
$\begin{array}{ll}--\kappa=2 / 3 & -\kappa=5 / 6 \\ --\kappa=1 & -\kappa=7 / 6\end{array}$
\end{abstract}
(b)

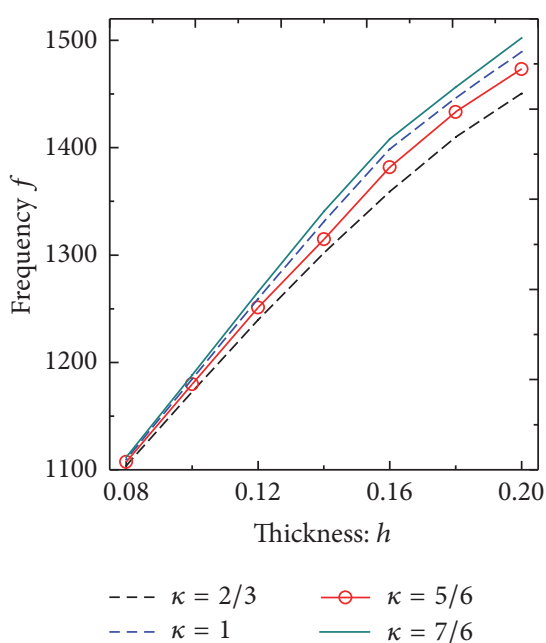

(e)

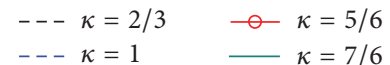

(c)

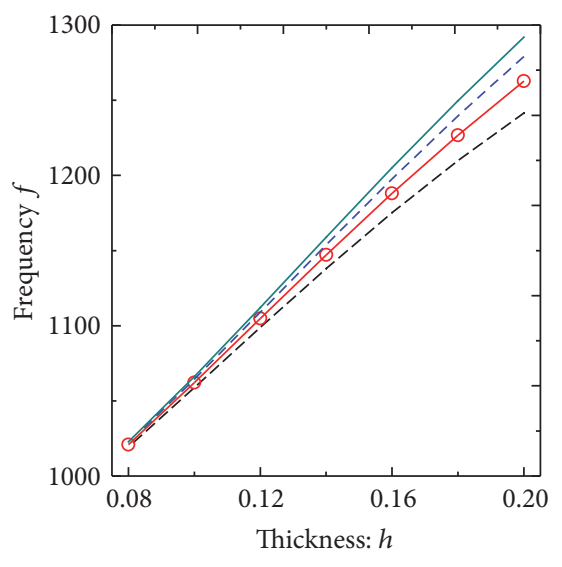

(f)

FIGURE 10: Fundamental frequency parameters versus thickness $h$ for the CCCC FG panels and shells with different shear correction factors: (a) cylindrical panels; (b) cylindrical shells; (c) conical panels; (d) conical shells; (e) spherical panels; (f) spherical shells.

$$
\begin{aligned}
& \cdot \frac{1}{A^{2} B} \frac{\partial B}{\partial \alpha}\left(\mathbf{U}^{T} \frac{\partial \Phi}{\partial \alpha}+\mathbf{U} \frac{\partial \Phi^{T}}{\partial \alpha}\right)+B_{66} \\
& \cdot \frac{A^{2}}{B^{2}}\left(\frac{\partial}{\partial \beta}\left(\frac{\mathbf{U}^{T}}{A}\right) \frac{\partial}{\partial \beta}\left(\frac{\Phi}{A}\right)+\frac{\partial}{\partial \beta}\left(\frac{\mathbf{U}}{A}\right) \frac{\partial}{\partial \beta}\left(\frac{\boldsymbol{\Phi}^{T}}{A}\right)\right) \\
&+\left.B_{11} \frac{1}{A^{2} B^{2}}\left(\frac{\partial B}{\partial \alpha}\right)^{2}\left(\mathbf{U}^{T} \boldsymbol{\Phi}+\mathbf{U} \boldsymbol{\Phi}^{T}\right)\right\} d S \\
& \mathbf{K}_{u \beta}=\iint\left\{B_{11} \frac{1}{A^{2} B} \frac{\partial A}{\partial \beta}\left(\frac{\partial \mathbf{U}^{T}}{\partial \alpha} \boldsymbol{\Theta}+\frac{\partial \mathbf{U}}{\partial \alpha} \boldsymbol{\Theta}^{T}\right)+B_{11} \frac{1}{A B^{2}}\right. \\
&+\frac{\partial B}{\partial \alpha}\left(\frac{\partial \Theta^{T}}{\partial \beta} \mathbf{U}+\frac{\partial \Theta}{\partial \beta} \mathbf{U}^{T}\right)+B_{12} \frac{1}{A B}\left(\frac{\partial \mathbf{U}^{T}}{\partial \alpha} \frac{\partial \Theta}{\partial \beta}\right. \\
&\left.+\frac{\partial \mathbf{U}}{\partial \alpha} \frac{\partial \Theta^{T}}{\partial \beta}\right)+B_{12} \frac{1}{A^{2} B^{2}} \frac{\partial A}{\partial \beta} \frac{\partial B}{\partial \alpha}\left(\mathbf{U}^{T} \boldsymbol{\Theta}+\mathbf{U} \Theta^{T}\right) \\
&+B_{66}\left(\frac{\partial}{\partial \beta}\left(\frac{\mathbf{U}^{T}}{A}\right) \frac{\partial}{\partial \alpha}\left(\frac{\boldsymbol{\Theta}}{B}\right)\right.
\end{aligned}
$$$$
\left.\left.+\frac{\partial}{\partial \beta}\left(\frac{\mathbf{U}}{A}\right) \frac{\partial}{\partial \alpha}\left(\frac{\boldsymbol{\Theta}^{T}}{B}\right)\right)\right\} d S
$$$$
\mathbf{K}_{v v}=\iint\left\{A_{11} \frac{1}{A B} \frac{\partial A}{\partial \beta} \mathbf{V}^{T} \mathbf{V}+A_{11} \frac{1}{B} \frac{\partial \mathbf{V}^{T}}{\partial \beta} \frac{\partial \mathbf{V}}{\partial \beta}+A_{66} \frac{B^{2}}{A^{2}}\right.
$$$$
\cdot \frac{\partial}{\partial \alpha}\left(\frac{\mathbf{V}^{T}}{B}\right) \frac{\partial}{\partial \alpha}\left(\frac{\mathbf{V}}{B}\right)+A_{12} \frac{1}{A B^{2}} \frac{\partial A}{\partial \beta}\left(\mathbf{V}^{T} \frac{\partial \mathbf{V}}{\partial \beta}\right.
$$$$
\left.\left.+\mathbf{V} \frac{\partial \mathbf{V}^{T}}{\partial \beta}\right)+\kappa A_{66} \frac{1}{R_{\beta}^{2}} \mathbf{V}^{T} \mathbf{V}\right\} d S
$$$$
+\iint\left\{k_{\alpha 0}^{u} \mathbf{V}^{T} \mathbf{V}\right\}_{\mid \alpha=0} d S_{0}+\iint\left\{k_{\alpha 1}^{u} \mathbf{V}^{T} \mathbf{V}\right\}_{\mid \alpha=L_{\varphi}} d S_{1}
$$$$
+\iint\left\{k_{\beta 0}^{u} \mathbf{V}^{T} \mathbf{V}\right\}_{\mid \beta=0} d S_{2}+\iint\left\{k_{\beta 1}^{u} \mathbf{V}^{T} \mathbf{V}\right\}_{\mid \beta=L_{\beta}} d S_{3}
$$$$
+\iint\left\{k_{u c}^{u}\left(\mathbf{V}_{\mid \beta=0}-\mathbf{V}_{\mid \beta=2 \pi}\right)^{T}\left(\mathbf{V}_{\mid \beta=0}-\mathbf{V}_{\mid \beta=2 \pi}\right)\right\} d S_{2}
$$ 

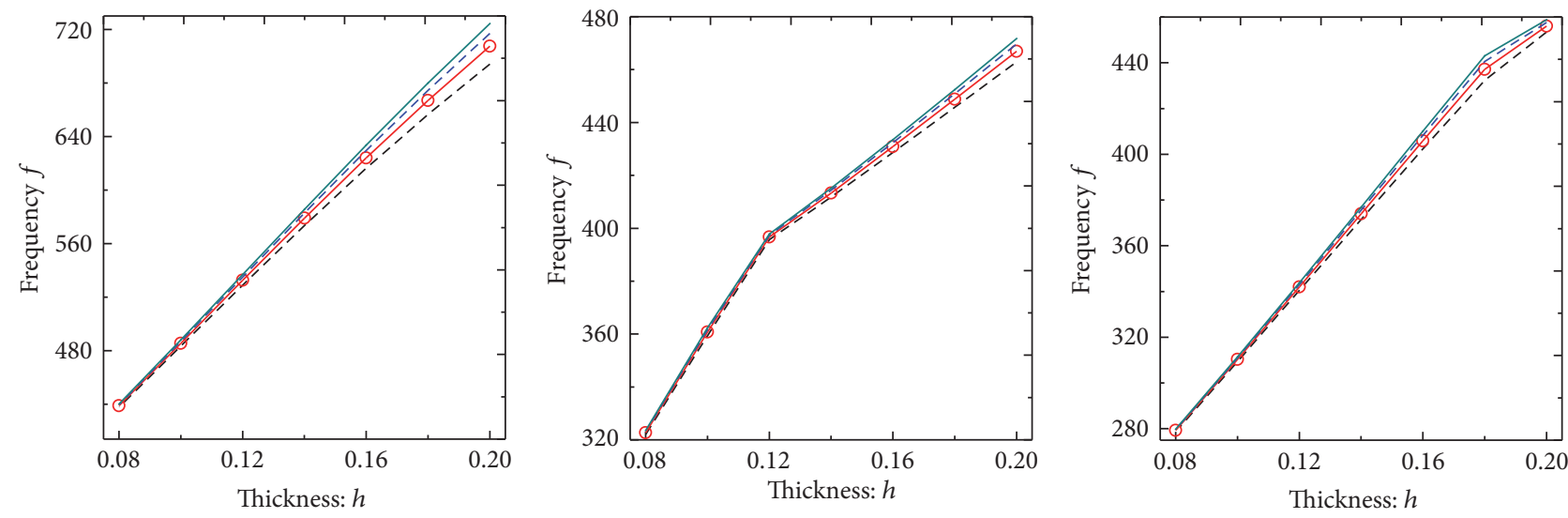

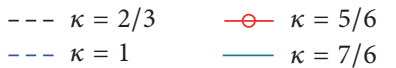

(a)

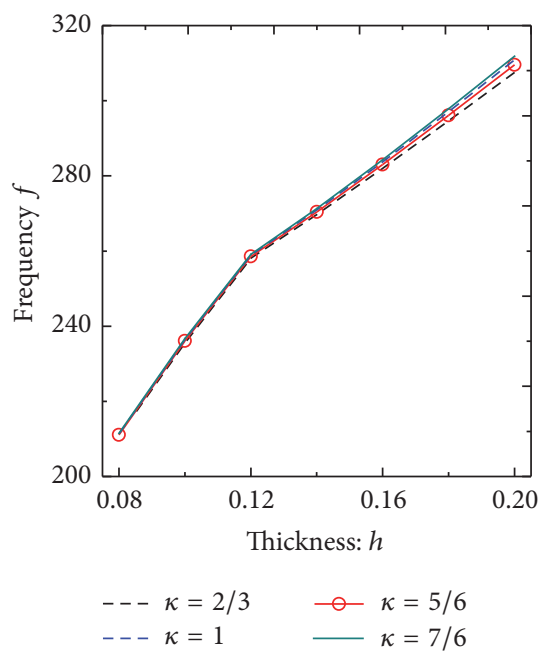

(d)

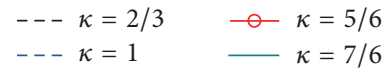

(b)

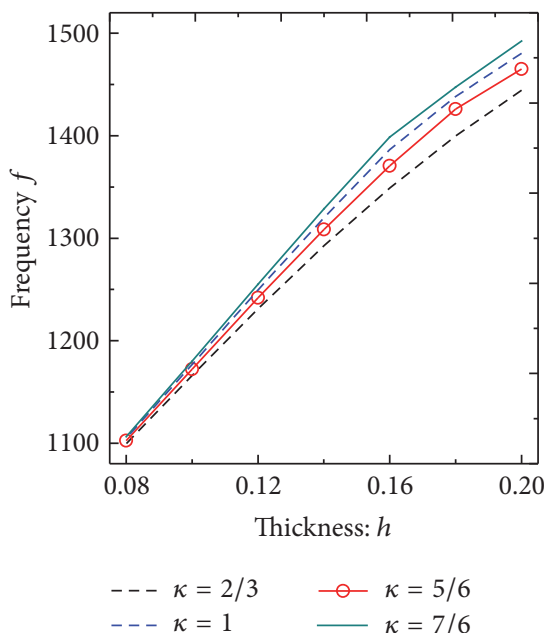

(e)

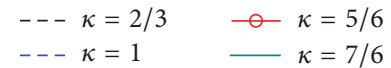

(c)

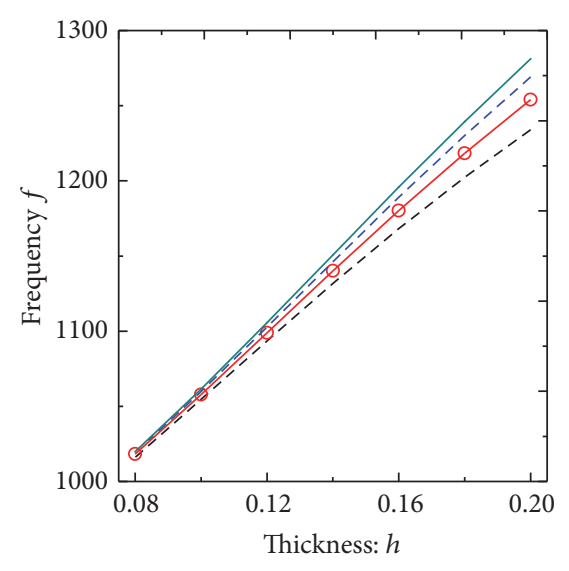

(f)

FIGURE 11: Fundamental frequency parameters versus thickness $h$ for the $\mathrm{E}^{3} \mathrm{E}^{3} \mathrm{E}^{3} \mathrm{E}^{3} \mathrm{FG}$ panels and shells with different shear correction factors: (a) cylindrical panels; (b) cylindrical shells; (c) conical panels; (d) conical shells; (e) spherical panels; (f) spherical shells.

$$
\begin{array}{cc}
\mathbf{K}_{v w}=\iint\left\{A_{11} \frac{1}{A B R_{\alpha}} \frac{\partial A}{\partial \alpha}\left(\mathbf{V}^{T} \mathbf{W}+\mathbf{W}^{T} \mathbf{V}\right)+A_{11}\right. & \left.+\boldsymbol{\Phi}^{T} \mathbf{V}\right)+B_{12} \frac{1}{A B}\left(\frac{\partial \mathbf{V}^{T}}{\partial \beta} \frac{\partial \Phi}{\partial \alpha}+\frac{\partial \Phi^{T}}{\partial \beta} \frac{\partial \mathbf{V}}{\partial \alpha}\right) \\
\cdot \frac{1}{B R_{\beta}}\left(\frac{\partial \mathbf{V}^{T}}{\partial \beta} \mathbf{W}+\mathbf{W}^{T} \frac{\partial \mathbf{V}}{\partial \beta}\right)+A_{12} \frac{1}{A B R_{\beta}} \frac{\partial A}{\partial \beta}\left(\mathbf{V}^{T} \mathbf{W}\right. & +B_{66}\left(\frac{\partial}{\partial \varphi}\left(\frac{\mathbf{V}^{T}}{B}\right) \frac{\partial}{\partial \beta}\left(\frac{\boldsymbol{\Phi}}{A}\right)\right. \\
\left.+\mathbf{W}^{T} \mathbf{V}\right)+A_{12} \frac{1}{B R_{\alpha}}\left(\frac{\partial \mathbf{V}^{T}}{\partial \beta} \mathbf{W}+\mathbf{W}^{T} \frac{\partial \mathbf{V}}{\partial \beta}\right)+\kappa A_{66} & \left.\left.+\frac{\partial}{\partial \varphi}\left(\frac{\mathbf{V}}{B}\right) \frac{\partial}{\partial \beta}\left(\frac{\boldsymbol{\Phi}^{T}}{A}\right)\right)\right\} d S \\
\left.\cdot \frac{1}{B R_{\beta}}\left(\frac{\partial \mathbf{W}^{T}}{\partial \beta} \mathbf{V}+\mathbf{V}^{T} \frac{\partial \mathbf{W}}{\partial \beta}\right)\right\} d S & \mathbf{K}_{v \beta}=\iint\left\{\kappa A_{66} \frac{1}{R_{\beta}}\left(\mathbf{V}^{T} \boldsymbol{\Theta}+\boldsymbol{\Theta}^{T} \mathbf{V}\right)+B_{11} \frac{1}{A^{2} B^{2}} \frac{\partial A}{\partial \beta}\right. \\
\mathbf{K}_{v \alpha}=\iint\left\{B_{11} \frac{1}{A^{2} B} \frac{\partial A}{\partial \beta}\left(\mathbf{V}^{T} \frac{\partial \Phi}{\partial \alpha}+\frac{\partial \Phi^{T}}{\partial \alpha} \mathbf{V}\right)+B_{11} \frac{1}{A B^{2}}\right. & \cdot \frac{\partial B}{\partial \alpha}\left(\mathbf{V}^{T} \boldsymbol{\Theta}+\Theta^{T} \mathbf{V}\right)+B_{11} \frac{1}{B^{2}}\left(\frac{\partial \mathbf{V}^{T}}{\partial \beta} \frac{\partial \Theta}{\partial \beta}+\frac{\partial \Theta^{T}}{\partial \beta} \frac{\partial \mathbf{V}}{\partial \beta}\right) \\
. \frac{\partial B}{\partial \alpha}\left(\Phi^{T} \frac{\partial \mathbf{V}}{\partial \alpha}+\frac{\partial \mathbf{V}^{T}}{\partial \alpha}\right)+B_{12} \frac{1}{A^{2} B^{2}} \frac{\partial A}{\partial \beta} \frac{\partial B}{\partial \alpha}\left(\mathbf{V}^{T} \boldsymbol{\Phi}\right. & +B_{12} \frac{1}{A B^{2}} \frac{\partial A}{\partial \beta}\left(\mathbf{V}^{T} \frac{\partial \Theta}{\partial \theta}+\frac{\partial \Theta^{T}}{\partial \theta} \mathbf{V}\right)+B_{12} \frac{1}{A B^{2}}
\end{array}
$$



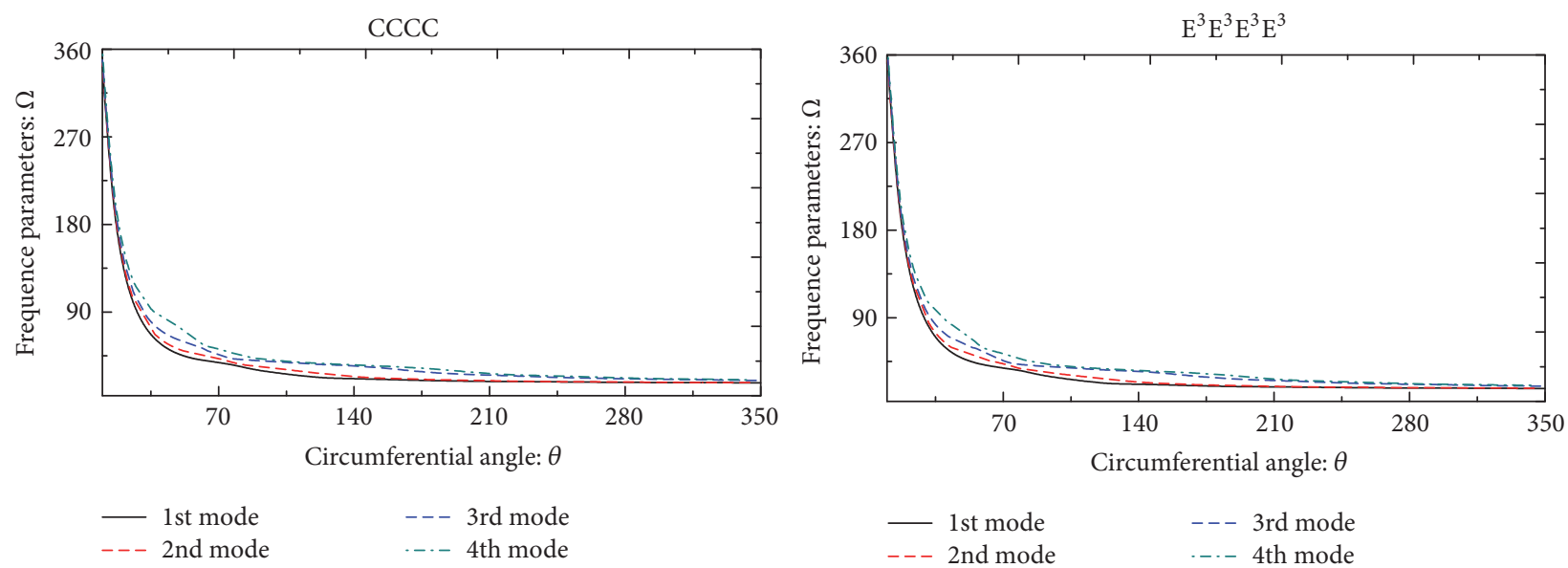

(a)
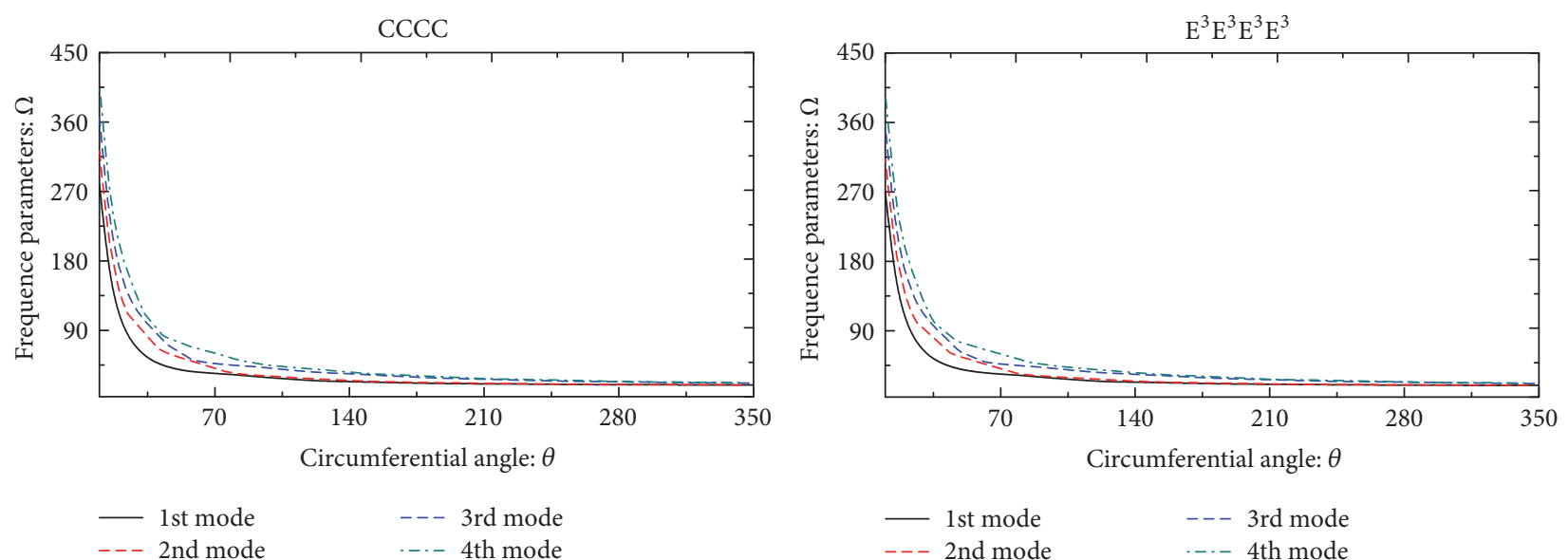

(b)
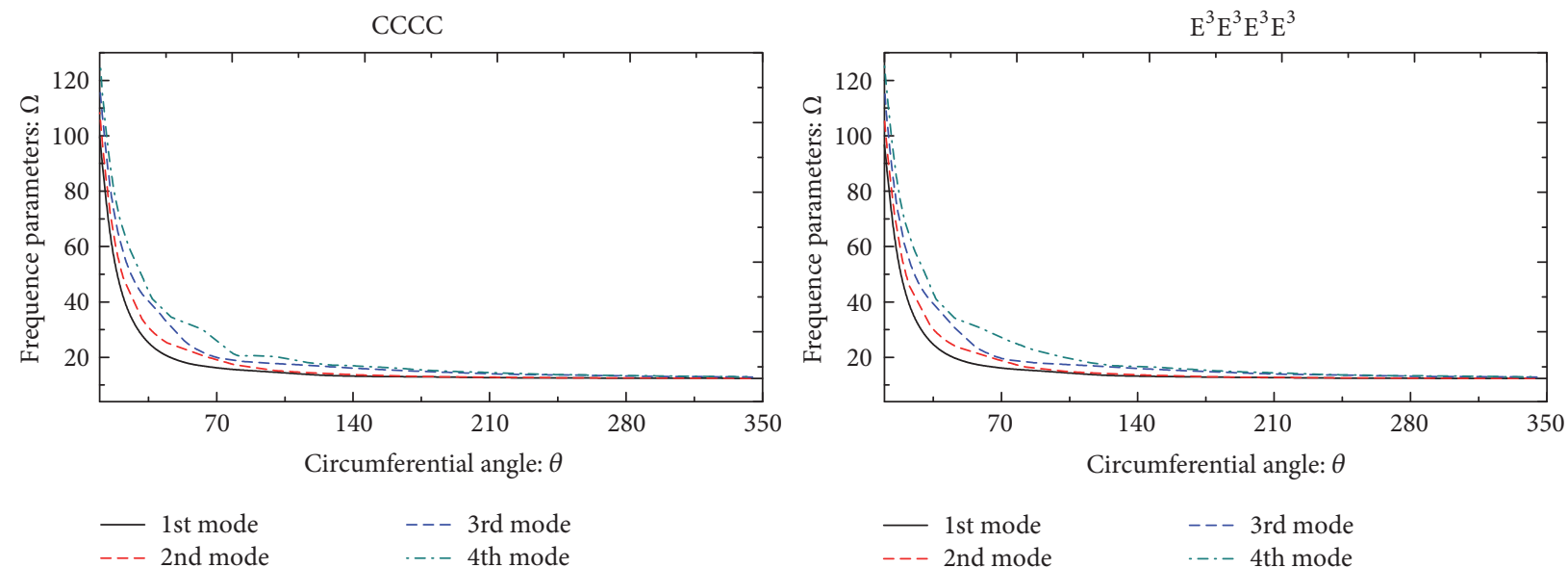

(c)

FIGURE 12: Variation of the first four frequency parameters $\Omega$ versus the circumferential angle for FG panels: (a) cylindrical panels; (b) conical panels; (c) spherical panels. 

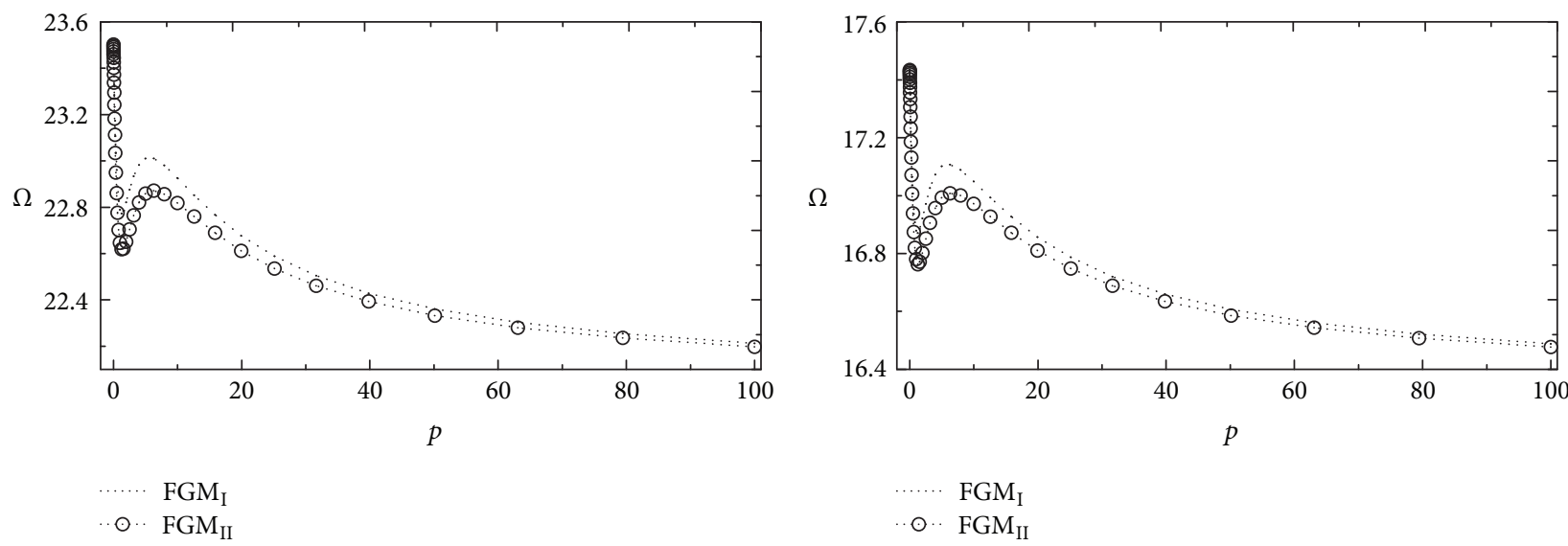

(a)
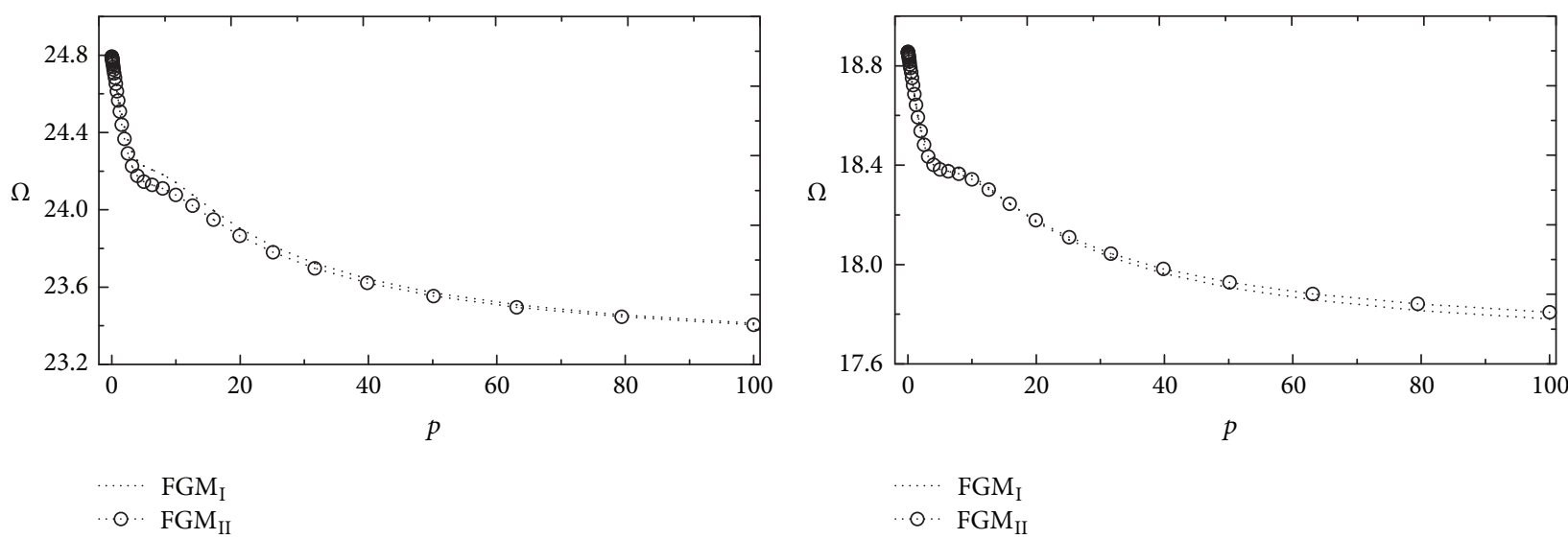

(c)
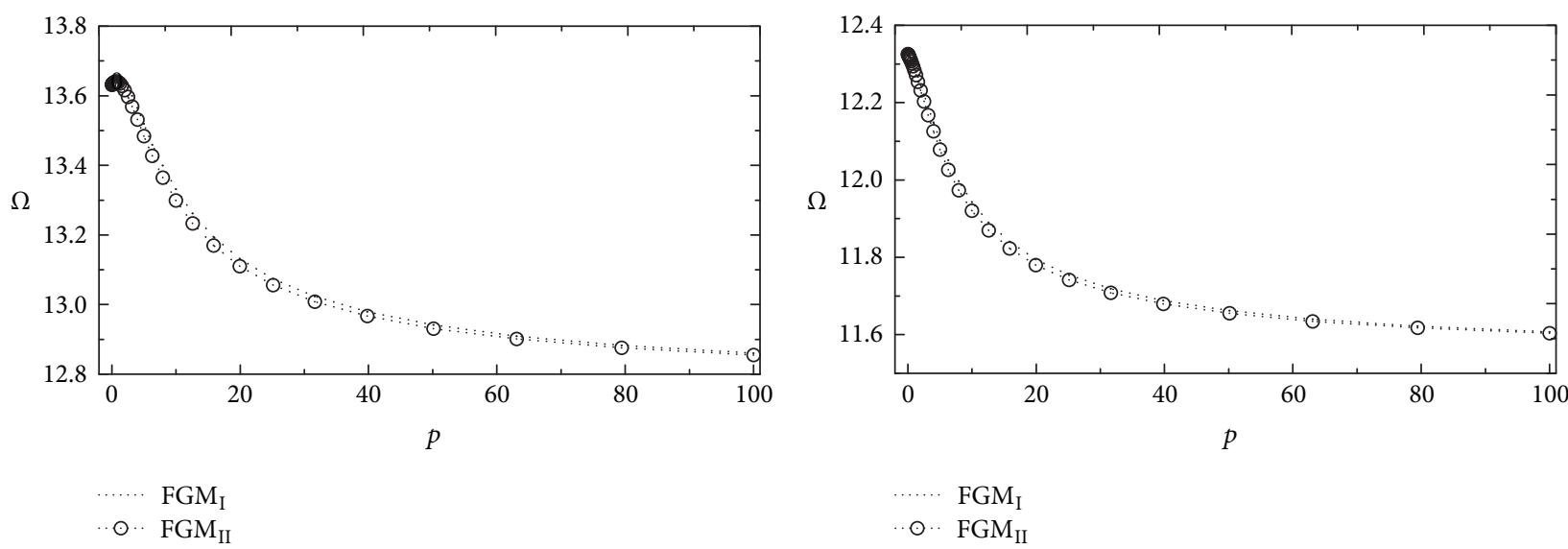

(e)

(f)

FIGURE 13: Variations of the first frequencies of the clamped supported $\mathrm{FGM}_{\mathrm{I}}$ and $\mathrm{FGM}_{\mathrm{II}}$ : (a) cylindrical panels; (b) cylindrical shells; (c) conical panels; (d) conical shells; (e) spherical panels; (f) spherical shells.

$$
\begin{array}{lc}
\cdot \frac{\partial A}{\partial \beta}\left(\boldsymbol{\Theta}^{T} \frac{\partial \mathbf{V}}{\partial \theta}+\frac{\partial \mathbf{V}^{T}}{\partial \theta} \boldsymbol{\Theta}\right)+B_{66} & \mathbf{K}_{w w}=\iint\left\{A_{11} \frac{1}{R_{\alpha}^{2}} \mathbf{W}^{T} \mathbf{W}+A_{11} \frac{1}{R_{\beta}^{2}} \mathbf{W}^{T} \mathbf{W}+\kappa A_{66} \frac{1}{A^{2}} \frac{\partial \mathbf{W}^{T}}{\partial \alpha}\right. \\
\cdot \frac{B^{2}}{A^{2}}\left(\frac{\partial}{\partial \alpha}\left(\frac{\mathbf{V}^{T}}{B}\right) \frac{\partial}{\partial \alpha}\left(\frac{\boldsymbol{\Theta}}{B}\right)\right. & \left.\frac{\partial \mathbf{W}}{\partial \alpha}+A_{12} \frac{1}{R_{\alpha} R_{\beta}} \mathbf{W}^{T} \mathbf{W}+\kappa A_{66} \frac{1}{B^{2}} \frac{\partial \mathbf{W}^{T}}{\partial \beta} \frac{\partial \mathbf{W}}{\partial \beta}\right\} d S \\
\left.\left.+\frac{\partial}{\partial \alpha}\left(\frac{\boldsymbol{\Theta}^{T}}{B}\right) \frac{\partial}{\partial \alpha}\left(\frac{\mathbf{V}}{B}\right)\right)\right\} d S & +\iint\left\{k_{\alpha 0}^{u} \mathbf{W}^{T} \mathbf{W}\right\}_{\mid \alpha=0} d S_{0}+\iint\left\{k_{\alpha 1}^{u} \mathbf{W}^{T} \mathbf{W}\right\}_{\mid \alpha=L_{\varphi}} d S_{1}
\end{array}
$$



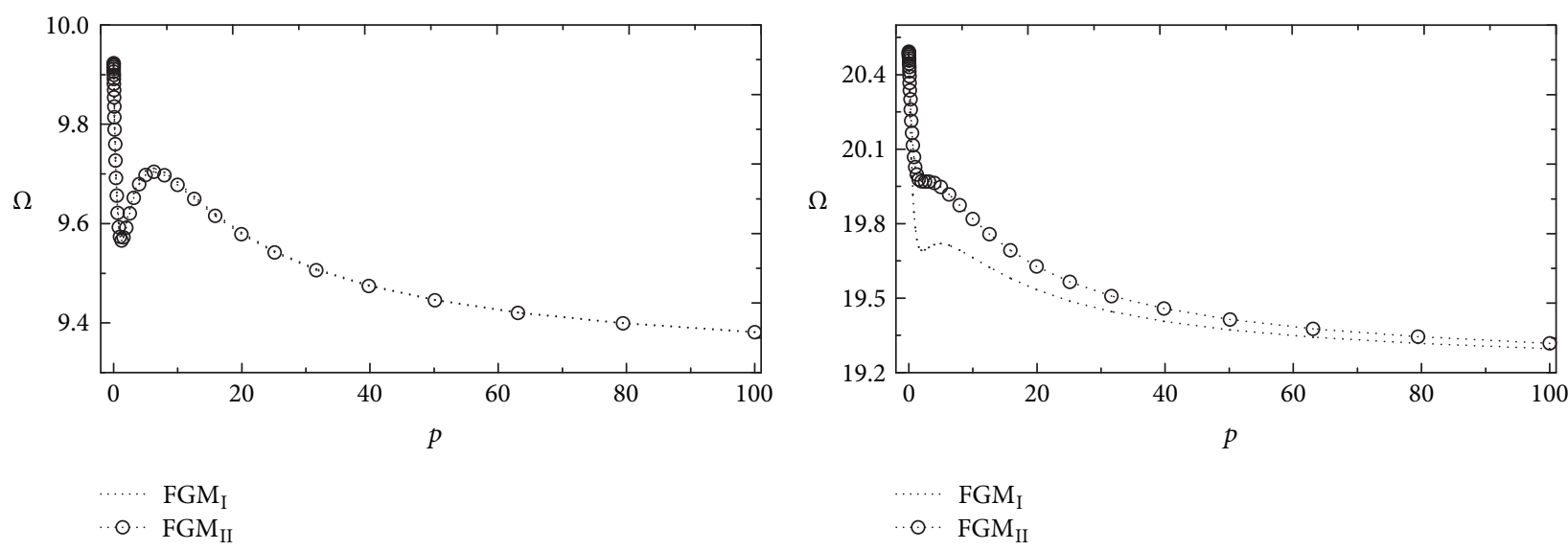

(a) $\mathrm{CFCF}$
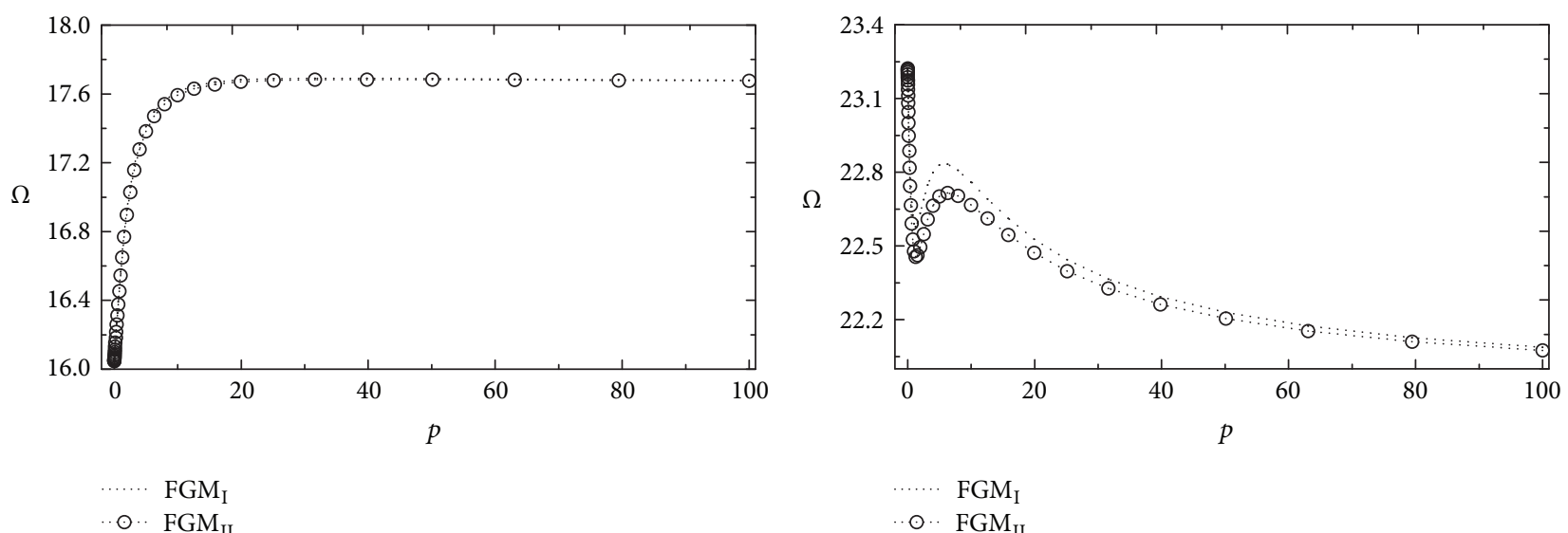

(c) $E^{1} E^{1} E^{1} E^{1}$

(d) $E^{3} E^{3} E^{3} E^{3}$

FIGURE 14: Variations of the first frequencies of the $\mathrm{FGM}_{\mathrm{I}}$ and $\mathrm{FGM}_{\mathrm{II}}$ cylindrical panels with different boundary conditions.

$$
\begin{aligned}
& +\iint\left\{k_{\beta 0}^{u} \mathbf{W}^{T} \mathbf{W}\right\}_{\mid \beta=0} d S_{2}+\iint\left\{k_{\beta 1}^{u} \mathbf{W}^{T} \mathbf{W}\right\}_{\mid \beta=L_{\beta}} d S_{3} \\
& +B_{12} \frac{1}{B R_{\alpha}}\left(\frac{\partial \Theta^{T}}{\partial \beta} \mathbf{W}+\frac{\partial \Theta}{\partial \beta} \mathbf{W}^{T}\right)+B_{12} \frac{1}{A B R_{\beta}} \frac{\partial A}{\partial \beta}\left(\Theta^{T} \mathbf{W}\right. \\
& +\iint\left\{k_{u c}^{u}\left(\mathbf{W}_{\mid \beta=0}-\mathbf{W}_{\mid \beta=2 \pi}\right)^{T}\left(\mathbf{W}_{\mid \beta=0}-\mathbf{W}_{\mid \beta=2 \pi}\right)\right\} d S_{2} \\
& \left.\left.+\boldsymbol{\Theta} \mathbf{W}^{T}\right)\right\} d S \\
& \mathbf{K}_{w \alpha}=\iint\left\{\kappa A_{66} \frac{1}{A}\left(\frac{\partial \mathbf{W}^{T}}{\partial \alpha} \boldsymbol{\Phi}+\frac{\partial \mathbf{W}}{\partial \alpha} \boldsymbol{\Phi}^{T}\right)+B_{11}\right. \\
& \cdot \frac{1}{A R_{\alpha}}\left(\frac{\partial \Phi^{T}}{\partial \alpha} \mathbf{W}+\frac{\partial \Phi}{\partial \alpha} \mathbf{W}^{T}\right)+B_{11} \frac{1}{A B R_{\beta}} \frac{\partial B}{\partial \alpha}\left(\Phi^{T} \mathbf{W}\right. \\
& \left.+\boldsymbol{\Phi} \mathbf{W}^{T}\right)+B_{12} \frac{1}{A B R_{\alpha}} \frac{\partial B}{\partial \alpha}\left(\boldsymbol{\Phi}^{T} \mathbf{W}+\boldsymbol{\Phi} \mathbf{W}^{T}\right)+B_{12} \\
& \left.\frac{1}{A R_{\beta}}\left(\frac{\partial \Phi^{T}}{\partial \alpha} \mathbf{W}+\frac{\partial \Phi}{\partial \alpha} \mathbf{W}^{T}\right)\right\} d S \\
& \mathbf{K}_{\alpha \alpha}=\iint\left\{\kappa A_{66} \boldsymbol{\Phi}^{T} \boldsymbol{\Phi}+D_{11} \frac{1}{A^{2}} \frac{\partial \boldsymbol{\Phi}^{T}}{\partial \alpha} \frac{\partial \boldsymbol{\Phi}}{\partial \alpha}+D_{11}\left(\frac{1}{A B} \frac{\partial B}{\partial \alpha}\right)^{2}\right. \\
& \cdot \boldsymbol{\Phi}^{T} \boldsymbol{\Phi}+D_{66} \frac{A^{2}}{B^{2}} \frac{\partial}{\partial \beta}\left(\frac{\boldsymbol{\Phi}^{T}}{A}\right) \frac{\partial}{\partial \beta}\left(\frac{\boldsymbol{\Phi}}{A}\right) \\
& \left.+A_{12}\left(\frac{1}{A^{2} B} \frac{\partial B}{\partial \alpha} \frac{\partial \boldsymbol{\Phi}^{T}}{\partial \alpha} \boldsymbol{\Phi}+\frac{1}{A^{2} B} \frac{\partial B}{\partial \alpha} \frac{\partial \Phi}{\partial \alpha} \Phi^{T}\right)\right\} d S \\
& +\iint\left\{k_{\alpha 0}^{u} \boldsymbol{\Phi}^{T} \boldsymbol{\Phi}\right\}_{\mid \alpha=0} d S_{0}+\iint\left\{k_{\alpha 1}^{u} \boldsymbol{\Phi}^{T} \boldsymbol{\Phi}\right\}_{\mid \alpha=L_{\varphi}} d S_{1} \\
& \mathbf{K}_{\omega \beta}=\iint\left\{\kappa A_{66} \frac{1}{B}\left(\frac{\partial \mathbf{W}^{T}}{\partial \beta} \boldsymbol{\Theta}+\frac{\partial \mathbf{W}}{\partial \beta} \boldsymbol{\Theta}^{T}\right)+B_{11} \frac{1}{A B R_{\alpha}}\right. \\
& +\iint\left\{k_{\beta 0}^{u} \boldsymbol{\Phi}^{T} \boldsymbol{\Phi}\right\}_{\mid \beta=0} d S_{2}+\iint\left\{k_{\beta 1}^{u} \boldsymbol{\Phi}^{T} \boldsymbol{\Phi}\right\}_{\mid \beta=L_{\beta}} d S_{3} \\
& \cdot \frac{\partial A}{\partial \beta}\left(\boldsymbol{\Theta}^{T} \mathbf{W}+\boldsymbol{\Theta} \mathbf{W}^{T}\right)+B_{11} \frac{1}{B R_{\beta}}\left(\frac{\partial \Theta^{T}}{\partial \beta} \mathbf{W}+\frac{\partial \Theta}{\partial \beta} \mathbf{W}^{T}\right) \\
& +\iint\left\{K_{\alpha c}^{\alpha}\left(\boldsymbol{\Phi}_{\mid \beta=0}-\boldsymbol{\Phi}_{\mid \beta=2 \pi}\right)^{T}\left(\boldsymbol{\Phi}_{\mid \beta=0}-\boldsymbol{\Phi}_{\mid \beta=2 \pi}\right)\right\} d S_{2}
\end{aligned}
$$




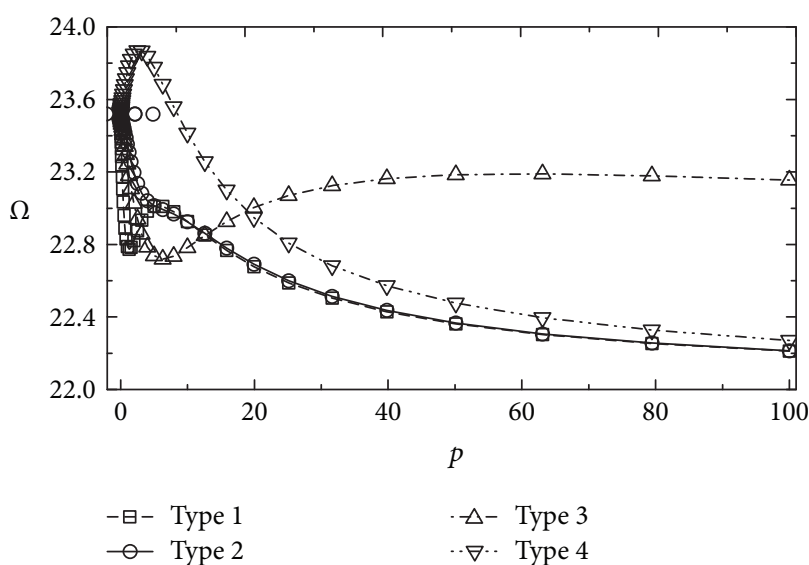

(a) Cylindrical panels

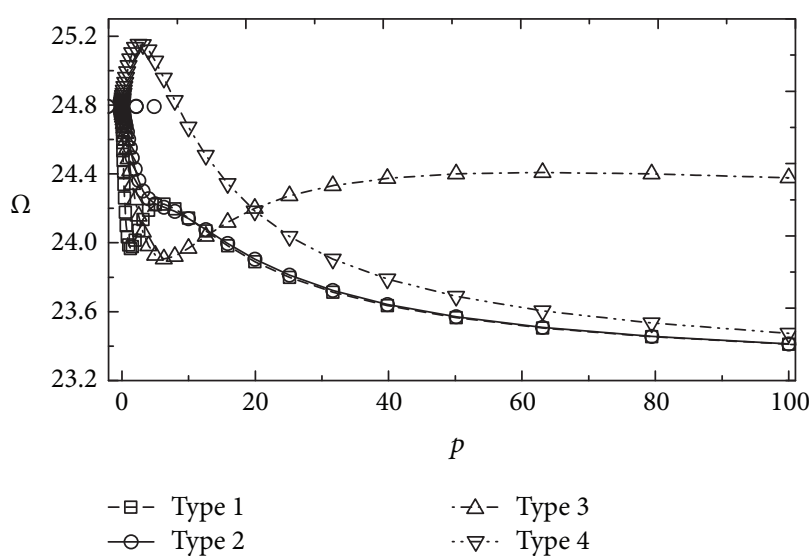

(c) Conical panels

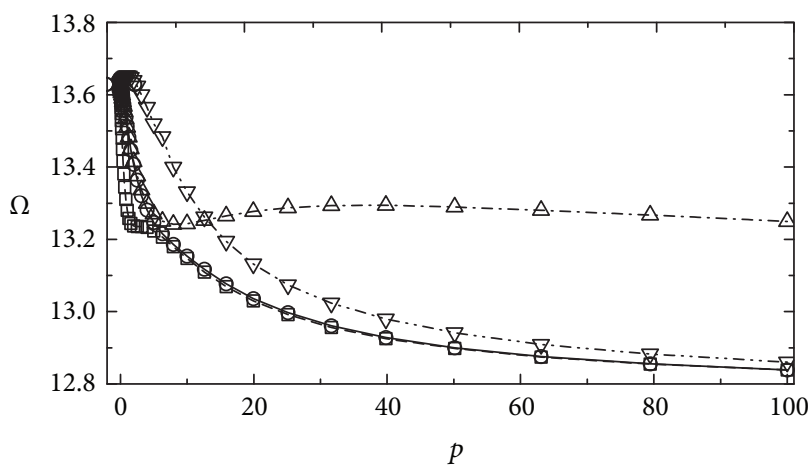

(e) Spherical panels
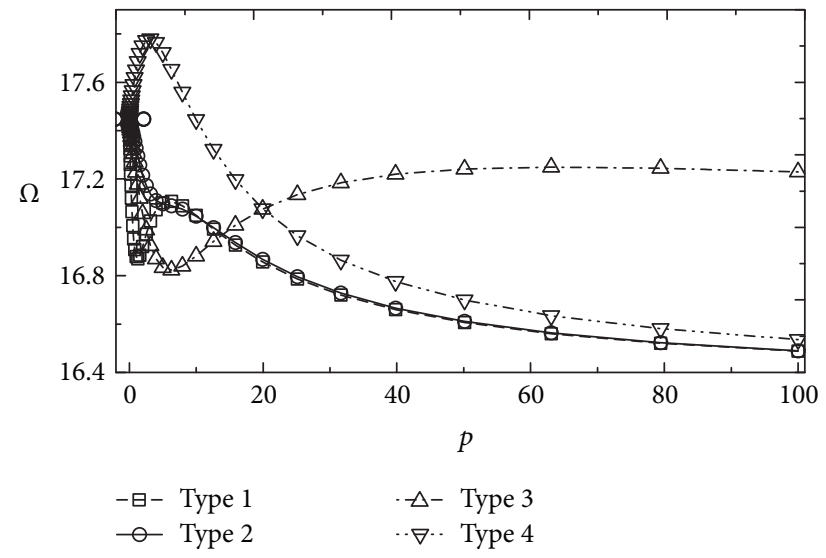

(b) Cylindrical shells

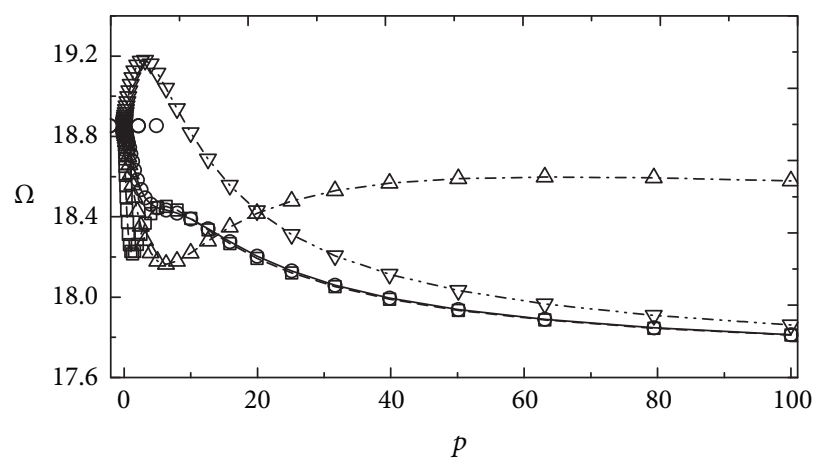

$\begin{array}{ll}-\bullet-\text { Type } 1 & -\triangle-\text { Type } 3 \\ - & -\nabla \cdot-\text { Type } 4\end{array}$

(d) Conical shells

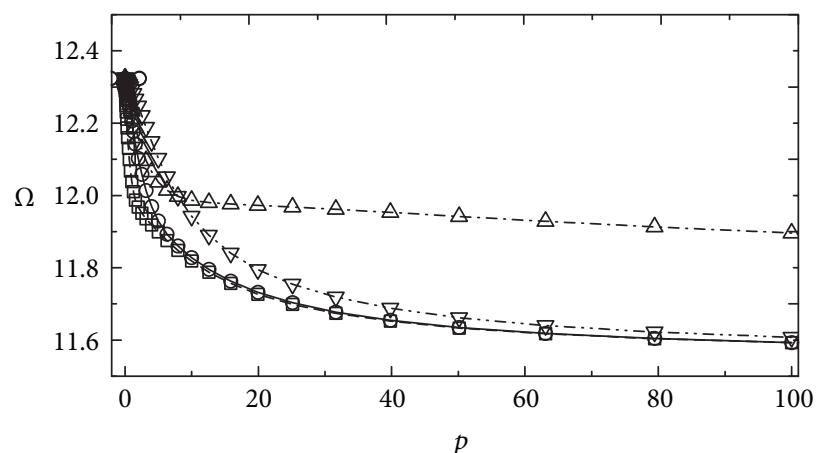

$$
\begin{array}{ll}
-\bullet-\text { Type } 1 & \cdot \triangle-\text { Type } 3 \\
-0 \text { Type } 2 & \cdot \cdot \nabla \cdot \cdot \text { Type } 4
\end{array}
$$

(f) Spherical shells

FIGURE 15: Variations of the first frequencies of the clamped supported FGM $\mathrm{I}_{\mathrm{I}}$ panels and shells with different material types: Type 1: $a=1$ and $b=c=0$; Type 2: $a=1, b=0.5$, and $c=2$; Type 3: $a=0, b=-0.5$, and $c=2$; Type 4: $a=1, b=1$, and $c=4$.

$$
\begin{array}{cc}
\mathbf{K}_{\alpha \beta}=\iint\left\{D_{11} \frac{1}{A^{2} B} \frac{\partial A}{\partial \beta}\left(\boldsymbol{\Theta}^{T} \frac{\partial \Phi}{\partial \alpha}+\boldsymbol{\Theta} \frac{\partial \boldsymbol{\Phi}^{T}}{\partial \alpha}\right)+D_{11} \frac{1}{A B^{2}}\right. & \left.+\frac{\partial \Theta}{\partial \beta} \frac{\partial \Phi^{T}}{\partial \alpha}\right)+D_{12} \frac{1}{A^{2} B^{2}} \frac{\partial A}{\partial \beta} \frac{\partial B}{\partial \alpha}\left(\boldsymbol{\Theta}^{T} \boldsymbol{\Phi}+\boldsymbol{\Phi}^{T} \boldsymbol{\Theta}\right) \\
\cdot \frac{\partial B}{\partial \alpha}\left(\boldsymbol{\Phi}^{T} \frac{\partial \boldsymbol{\Theta}}{\partial \beta}+\boldsymbol{\Phi} \frac{\partial \boldsymbol{\Theta}^{T}}{\partial \beta}\right)+D_{12} \frac{1}{A B}\left(\frac{\partial \Phi}{\partial \alpha} \frac{\partial \boldsymbol{\Theta}^{T}}{\partial \beta}\right. & +D_{66}\left(\frac{\partial}{\partial \alpha}\left(\frac{\boldsymbol{\Theta}^{T}}{B}\right) \frac{\partial}{\partial \beta}\left(\frac{\boldsymbol{\Phi}}{A}\right)\right.
\end{array}
$$




$$
\begin{aligned}
& \left.\left.+\frac{\partial}{\partial \alpha}\left(\frac{\boldsymbol{\Theta}}{B}\right) \frac{\partial}{\partial \beta}\left(\frac{\boldsymbol{\Phi}^{T}}{A}\right)\right)\right\} d S \\
& \mathbf{K}_{\beta \beta}=\iint\left\{\kappa A_{66} \boldsymbol{\Theta}^{T} \boldsymbol{\Theta}+D_{11} \frac{R_{\varphi}}{R_{0}} \frac{1}{A B} \frac{\partial A}{\partial \beta} \boldsymbol{\Theta}^{T} \boldsymbol{\Theta}+D_{11} \frac{1}{B} \frac{\partial \boldsymbol{\Theta}^{T}}{\partial \beta}\right. \\
& \cdot \frac{\partial \Theta}{\partial \beta}+D_{66} \frac{B^{2}}{A^{2}} \frac{\partial}{\partial \alpha}\left(\frac{\Theta^{T}}{B}\right) \frac{\partial}{\partial \alpha}\left(\frac{\Theta}{B}\right)+D_{12} \frac{1}{A B^{2}} \\
& \left.\frac{\partial A}{\partial \beta}\left(\boldsymbol{\Theta}^{T} \frac{\partial \Theta}{\partial \beta}+\Theta \frac{\partial \Theta^{T}}{\partial \beta}\right)\right\} d S \\
& +\iint\left\{k_{\alpha 0}^{u} \boldsymbol{\Theta}^{T} \boldsymbol{\Theta}\right\}_{\mid \alpha=0} d S_{0}+\iint\left\{k_{\alpha 1}^{u} \boldsymbol{\Theta}^{T} \boldsymbol{\Theta}\right\}_{\mid \alpha=L_{\varphi}} d S_{1} \\
& +\iint\left\{k_{\beta 0}^{u} \boldsymbol{\Theta}^{T} \boldsymbol{\Theta}\right\}_{\mid \beta=0} d S_{2}+\iint\left\{k_{\beta 1}^{u} \boldsymbol{\Theta}^{T} \boldsymbol{\Theta}\right\}_{\mid \beta=L_{\beta}} d S_{3} \\
& +\iint\left\{K_{\beta c}^{\beta}\left(\boldsymbol{\Theta}_{\mid \beta=0}-\boldsymbol{\Theta}_{\mid \beta=2 \pi}\right)^{T}\left(\boldsymbol{\Theta}_{\mid \beta=0}-\boldsymbol{\Theta}_{\mid \beta=2 \pi}\right)\right\} d S_{2} \\
& \mathbf{M}_{u u}=\iint\left\{I_{0} \mathbf{U}^{T} \mathbf{U}\right\} d S \\
& \mathbf{M}_{v v}=\iint\left\{I_{0} \mathbf{V}^{T} \mathbf{V}\right\} d S \\
& \mathbf{M}_{w w}=\iint\left\{I_{0} \mathbf{W}^{T} \mathbf{W}\right\} d S \\
& \mathbf{M}_{\alpha \alpha}=\iint\left\{I_{2} \boldsymbol{\Phi}^{T} \boldsymbol{\Phi}\right\} d S \\
& \mathbf{M}_{\beta \beta}=\iint\left\{I_{2} \boldsymbol{\Theta}^{T} \boldsymbol{\Theta}\right\} d S \\
& \mathbf{M}_{u \alpha}=\iint\left\{I_{1}\left(\mathbf{U}^{T} \boldsymbol{\Phi}+\mathbf{U} \boldsymbol{\Phi}^{T}\right)\right\} d S, \\
& \mathbf{M}_{v \beta}=\iint\left\{I_{1}\left(\mathbf{V}^{T} \boldsymbol{\Theta}+\mathbf{V} \boldsymbol{\Phi}^{T}\right)\right\} d S \\
& \mathbf{H}_{u} \\
& =\left\{\begin{array}{c}
A_{00}^{u}, A_{01}^{u}, \ldots, A_{m^{\prime} 0}^{u}, A_{m^{\prime}}^{u}, \ldots, A_{m^{\prime} n^{\prime}}^{u}, \ldots, A_{M N}^{u}, \\
a_{0}^{1}, \ldots, a_{M}^{1}, a_{0}^{2}, \ldots, a_{M}^{2}, b_{0}^{1}, \ldots, b_{N}^{1}, b_{0}^{2}, \ldots, b_{N}^{2}
\end{array}\right\} e^{j \omega t} \\
& \mathbf{H}_{v}=\left\{\begin{array}{l}
B_{00}^{v}, B_{01}^{v}, \ldots, B_{m^{\prime} 0}^{v}, B_{m^{\prime} 1}^{v}, \ldots, B_{m^{\prime} n^{\prime}}^{v}, \ldots, B_{M N}^{v}, \\
c_{0}^{1}, \ldots, c_{M}^{1}, c_{0}^{2}, \ldots, c_{M}^{2}, d_{0}^{1}, \ldots, d_{N}^{1}, d_{0}^{2}, \ldots, d_{N}^{2}
\end{array}\right\} e^{j \omega t} \\
& \mathbf{H}_{w}=\left\{\begin{array}{l}
C_{00}^{w}, C_{01}^{w}, \ldots, C_{m^{\prime} 0}^{w}, C_{m^{\prime} 1}^{w}, \ldots, C_{m^{\prime} n^{\prime}}^{w}, \ldots, C_{M N}^{w}, \\
e_{0}^{1}, \ldots, e_{M}^{1}, e_{0}^{2}, \ldots, e_{M}^{2}, e f_{0}^{1}, \ldots, f_{N}^{1}, f_{0}^{2}, \ldots, f_{N}^{2}
\end{array}\right\} e^{j \omega t} \\
& \mathbf{H}_{\alpha} \\
& =\left\{\begin{array}{c}
D_{00}^{\alpha}, D_{01}^{\alpha}, \ldots, D_{m^{\prime} 0}^{\alpha}, D_{m^{\prime} 1}^{\alpha}, \ldots, D_{m^{\prime} h^{\prime}}^{\alpha}, \ldots, D_{M N}^{\alpha} \\
g_{0}^{1}, \ldots, g_{M}^{1}, g_{0}^{2}, \ldots, g_{M}^{2}, h_{0}^{1}, \ldots, h_{N}^{1}, h_{0}^{2}, \ldots, h_{N}^{2}
\end{array}\right\} e^{j \omega t} \\
& \mathbf{H}_{\beta}=\left\{\begin{array}{l}
E_{00}^{\beta}, E_{01}^{\beta}, \ldots, E_{m^{\prime} 0}^{\beta}, E_{m^{\prime} 1}^{\beta}, \ldots, E_{m^{\prime} n^{\prime}}^{\beta}, \ldots, E_{M N}^{\beta}, \\
k_{0}^{1}, \ldots, k_{M}^{1}, k_{0}^{2}, \ldots, k_{M}^{2}, q_{0}^{1}, \ldots, q_{N}^{1}, q_{0}^{2}, \ldots, q_{N}^{2}
\end{array}\right\} e^{j \omega t},
\end{aligned}
$$

where

$\mathrm{U}$

$$
\begin{aligned}
& =\left\{\begin{array}{c}
\cos \lambda_{0} \alpha, \ldots, \cos \lambda_{0} \alpha \cos \lambda_{n} \beta, \ldots, \cos \lambda_{M} \alpha \cos \lambda_{N} \beta, \\
\chi_{1}(\beta), \ldots, \chi_{1}(\beta) \cos \lambda_{m} \alpha, \ldots, \chi_{2}(\beta) \cos \lambda_{M} \alpha, \\
\zeta_{1}(\alpha), \ldots, \zeta_{1}(\alpha) \cos \lambda_{n} \beta, \ldots, \zeta_{2}(\alpha) \cos \lambda_{N} \beta,
\end{array}\right\} \\
& \mathbf{V}=\mathbf{W}=\boldsymbol{\Phi}=\boldsymbol{\Theta}=\mathbf{U}
\end{aligned}
$$

\section{Conflicts of Interest}

The authors declare that they have no conflicts of interest.

\section{Acknowledgments}

This study was funded by National Natural Science Foundation of China (nos. 51209052 and 61502116), National Key Research and Development Program of China (2016YFC0303406), Assembly Advanced Research Fund of China (6140210020105), Major Innovation Projects of High Technology Ship Funds of Ministry of Industry and Information of China, High Technology Ship Funds of Ministry of Industry and Information Technology of China, Fundamental Research Funds for the Central Universities (HEUCFD1515, HEUCFM170113), China Postdoctoral Science Foundation (2014M552661), and the Fundamental Research Funds for the Central Universities (HEUCFM160601).

\section{References}

[1] F. Tornabene, "Free vibration analysis of functionally graded conical, cylindrical shell and annular plate structures with a four-parameter power-law distribution," Computer Methods Applied Mechanics and Engineering, vol. 198, no. 37-40, pp. 29112935, 2009.

[2] F. Tornabene and E. Viola, "Free vibrations of four-parameter functionally graded parabolic panels and shells of revolution," European Journal of Mechanics - A/Solids, vol. 28, no. 5, pp. 9911013, 2009.

[3] F. Tornabene and E. Viola, "Free vibration analysis of functionally graded panels and shells of revolution," Meccanica, vol. 44, no. 3, pp. 255-281, 2009.

[4] X. Zhao and K. M. Liew, "An element-free analysis of mechanical and thermal buckling of functionally graded conical shell panels," International Journal for Numerical Methods in Engineering, vol. 86, no. 3, pp. 269-285, 2011.

[5] X. Zhao and K. M. Liew, "Free vibration analysis of functionally graded conical shell panels by a meshless method," Composite Structures, vol. 93, no. 2, pp. 649-664, 2011.

[6] B. S. Aragh and H. Hedayati, "Static response and free vibration of two-dimensional functionally graded metal/ceramic open cylindrical shells under various boundary conditions," Acta Mechanica, vol. 223, no. 2, pp. 309-330, 2012.

[7] Z. Su, G. Jin, S. Shi, and T. Ye, "A unified accurate solution for vibration analysis of arbitrary functionally graded spherical shell segments with general end restraints," Composite Structures, vol. 111, no. 1, pp. 271-284, 2014.

[8] Z. Su, G. Jin, and T. Ye, "Free vibration analysis of moderately thick functionally graded open shells with general boundary 
conditions," Composite Structures, vol. 117, no. 1, pp. 169-186, 2014.

[9] Z. Su, G. Jin, S. Shi, T. Ye, and X. Jia, "A unified solution for vibration analysis of functionally graded cylindrical, conical shells and annular plates with general boundary conditions," International Journal of Mechanical Sciences, vol. 80, pp. 62-80, 2014.

[10] A. H. Sofiyev, "The buckling of functionally graded truncated conical shells under dynamic axial loading," Journal of Sound and Vibration, vol. 305, no. 4-5, pp. 808-826, 2007.

[11] A. H. Sofiyev and N. Kuruoglu, "Vibration analysis of FGM truncated and complete conical shells resting on elastic foundations under various boundary conditions," Journal of Engineering Mathematics, vol. 77, pp. 131-145, 2012.

[12] A. H. Sofiyev and N. Kuruoglu, "On a problem of the vibration of functionally graded conical shells with mixed boundary conditions," Composites Part B: Engineering, vol. 70, pp. 122-130, 2015.

[13] A. H. Sofiyev and E. Schnack, "The vibration analysis of FGM truncated conical shells resting on two-parameter elastic foundations," Mechanics of Advanced Materials and Structures, vol. 19, no. 4, pp. 241-249, 2012.

[14] Y.-W. Kim, "Free vibration analysis of FGM cylindrical shell partially resting on Pasternak elastic foundation with an oblique edge," Composites Part B: Engineering, vol. 70, pp. 263-276, 2015.

[15] G. Jin, X. Xie, and Z. Liu, “The Haar wavelet method for free vibration analysis of functionally graded cylindrical shells based on the shear deformation theory," Composite Structures, vol. 108, no. 1, pp. 435-448, 2014.

[16] X. Xie, H. Zheng, and G. Jin, "Free vibration of four-parameter functionally graded spherical and parabolic shells of revolution with arbitrary boundary conditions," Composites Part B: Engineering, vol. 77, pp. 59-73, 2015.

[17] S. Hosseini-Hashemi, M. R. Ilkhani, and M. Fadaee, "Identification of the validity range of Donnell and Sanders shell theories using an exact vibration analysis of functionally graded thick cylindrical shell panel," Acta Mechanica, vol. 223, no. 5, pp. 11011118, 2012.

[18] Z. Iqbal, M. N. Naeem, and N. Sultana, "Vibration characteristics of FGM circular cylindrical shells using wave propagation approach," Acta Mechanica, vol. 208, no. 3-4, pp. 237-248, 2009.

[19] A. G. Shah, T. Mahmood, M. N. Naeem, Z. Iqbal, and S. H. Arshad, "Vibrations of functionally graded cylindrical shells based on elastic foundations," Acta Mechanica, vol. 211, no. 34, pp. 293-307, 2010.

[20] C. T. Loy, K. Y. Lam, and J. N. Reddy, "Vibration of functionally graded cylindrical shells," International Journal of Mechanical Sciences, vol. 41, no. 3, pp. 309-324, 1999.

[21] S. C. Pradhan, C. T. Loy, K. Y. Lam, and J. N. Reddy, "Vibration characteristics of functionally graded cylindrical shells under various boundary conditions," Applied Acoustics, vol. 61, pp. 111129,2000

[22] M. M. Najafizadeh and M. R. Isvandzibaei, "Vibration of functionally graded cylindrical shells based on higher order shear deformation plate theory with ring support," Acta Mechanica, vol. 191, no. 1-2, pp. 75-91, 2007.

[23] M. N. Naeem, S. H. Arshad, and C. B. Sharma, "The Ritz formulation applied to the study of the vibration frequency characteristics of functionally graded circular cylindrical shells," Proceedings of the Institution of Mechanical Engineers, Part C: Journal of Mechanical Engineering Science, vol. 224, no. 1, pp. 43-54, 2010.
[24] A. Taghvaeipour, M. Bonakdar, and M. T. Ahmadian, "Application of a new cylindrical element formulation in finite element structural analysis of FGM hollow cylinders," Finite Elements in Analysis and Design, vol. 50, pp. 1-7, 2012.

[25] S. Pradyumna and J. N. Bandyopadhyay, "Free vibration analysis of functionally graded curved panels using a higher-order finite element formulation," Journal of Sound and Vibration, vol. 318, no. 1-2, pp. 176-192, 2008.

[26] A. M. Neves, A. J. Ferreira, E. Carrera et al., "Free vibration analysis of functionally graded shells by a higher-order shear deformation theory and radial basis functions collocation, accounting for through-the-thickness deformations," European Journal of Mechanics - A/Solids, vol. 37, pp. 24-34, 2013.

[27] Y. Qu, X. Long, G. Yuan, and G. Meng, "A unified formulation for vibration analysis of functionally graded shells of revolution with arbitrary boundary conditions," Composites Part B: Engineering, vol. 50, pp. 381-402, 2013.

[28] H. Santos, C. M. Mota Soares, C. A. Mota Soares, and J. N. Reddy, "A semi-analytical finite element model for the analysis of cylindrical shells made of functionally graded materials under thermal shock," Composite Structures, vol. 86, no. 1-3, pp. 10-21, 2008.

[29] H. Santos, C. M. Mota Soares, C. A. Mota Soares, and J. N. Reddy, "A semi-analytical finite element model for the analysis of cylindrical shells made of functionally graded materials," Composite Structures, vol. 91, no. 4, pp. 427-432, 2009.

[30] S. S. Vel, "Exact elasticity solution for the vibration of functionally graded anisotropic cylindrical shells," Composite Structures, vol. 92, no. 11, pp. 2712-2727, 2010.

[31] W. L. Li, "Free vibrations of beams with general boundary conditions," Journal of Sound and Vibration, vol. 237, no. 4, pp. 709-725, 2000.

[32] W. L. Li, "Comparison of fourier sine and cosine series expansions for beams with arbitrary boundary conditions," Journal of Sound and Vibration, vol. 255, no. 1, pp. 185-194, 2003.

[33] D. Shi, Q. Wang, X. Shi, and F. Pang, "A series solution for the in-plane vibration analysis of orthotropic rectangular plates with non-uniform elastic boundary constraints and internal line supports," Archive of Applied Mechanics, vol. 85, no. 1, pp. 51-73, 2015.

[34] D. Shi, Q. Wang, X. Shi, and F. Pang, "An accurate solution method for the vibration analysis of Timoshenko beams with general elastic supports," Proceedings of the Institution of Mechanical Engineers, Part C: Journal of Mechanical Engineering Science, 2014.

[35] D. Shao, F. Hu, Q. Wang, F. Pang, and S. Hu, "Transient response analysis of cross-ply composite laminated rectangular plates with general boundary restraints by the method of reverberation ray matrix," Composite Structures, vol. 152, pp. 168-182, 2016.

[36] D. Shao, S. Hu, Q. Wang, and F. Pang, "A unified analysis for the transient response of composite laminated curved beam with arbitrary lamination schemes and general boundary restraints," Composite Structures, vol. 154, pp. 507-526, 2016.

[37] D. Shi, Y. Zhao, Q. Wang, X. Teng, and F. Pang, "A unified spectro-geometric-ritz method for vibration analysis of open and closed shells with arbitrary boundary conditions," Shock and Vibration, vol. 2016, Article ID 4097123, 30 pages, 2016.

[38] Q. Wang, D. Shi, and Q. Liang, "Free vibration analysis of axially loaded laminated composite beams with general boundary conditions by using a modified Fourier-Ritz approach," Journal of Composite Materials, vol. 50, no. 15, pp. 2111-2135, 2016. 
[39] Q. Wang, D. Shi, Q. Liang, and F. e Ahad, "A unified solution for free in-plane vibration of orthotropic circular, annular and sector plates with general boundary conditions," Applied Mathematical Modelling: Simulation and Computation for Engineering and Environmental Systems, vol. 40, no. 21-22, pp. 9228-9253, 2016.

[40] Q. Wang, D. Shi, Q. Liang, and X. Shi, "A unified solution for vibration analysis of functionally graded circular, annular and sector plates with general boundary conditions," Composites Part B: Engineering, vol. 88, pp. 264-294, 2016.

[41] D. Shao, S. Hu, Q. Wang, and F. Pang, "Free vibration of refined higher-order shear deformation composite laminated beams with general boundary conditions," Composites Part B: Engineering, vol. 108, pp. 75-90, 2017.

[42] D. Shao, S. Hu, Q. Wang, and F. Pang, "An enhanced reverberation-ray matrix approach for transient response analysis of composite laminated shallow shells with general boundary conditions," Composite Structures, vol. 162, pp. 133-155, 2017.

[43] Q. Wang, F. Pang, B. Qin, and Q. Liang, "A unified formulation for free vibration of functionally graded carbon nanotube reinforced composite spherical panels and shells of revolution with general elastic restraints by means of the Rayleigh-Ritz method," Polymer Composites, 2017.

[44] Q. Wang, B. Qin, D. Shi, and Q. Liang, "A semi-analytical method for vibration analysis of functionally graded carbon nanotube reinforced composite doubly-curved panels and shells of revolution," Composite Structures, vol. 174, pp. 87-109, 2017.

[45] Q. Wang, D. Shi, Q. Liang, and F. Pang, "Free vibrations of composite laminated doubly-curved shells and panels of revolution with general elastic restraints," Applied Mathematical Modelling, vol. 46, pp. 227-262, 2017.

[46] Q. Wang, D. Shi, Q. Liang, and F. Pang, "Free vibration of four-parameter functionally graded moderately thick doublycurved panels and shells of revolution with general boundary conditions," Applied Mathematical Modelling: Simulation and Computation for Engineering and Environmental Systems, vol. 42, pp. 705-734, 2017.

[47] H. Zhang, D. Shi, and Q. Wang, "An improved Fourier series solution for free vibration analysis of the moderately thick laminated composite rectangular plate with non-uniform boundary conditions," International Journal of Mechanical Sciences, vol. 121, pp. 1-20, 2017.

[48] Y. Zhou, Q. Wang, D. Shi, Q. Liang, and Z. Zhang, "Exact solutions for the free in-plane vibrations of rectangular plates with arbitrary boundary conditions," International Journal of Mechanical Sciences, vol. 130, pp. 1-10, 2017.

[49] G. Jin, S. Shi, Z. Su, S. Li, and Z. Liu, "A modified Fourier-Ritz approach for free vibration analysis of laminated functionally graded shallow shells with general boundary conditions," International Journal of Mechanical Sciences, vol. 93, pp. 256-269, 2015.

[50] F. Gruttmann and W. Wagner, "Shear correction factors in timoshenko's beam theory for arbitrary shaped cross-sections," Computational Mechanics, vol. 27, no. 3, pp. 199-207, 2001.

[51] Z. Su, G. Jin, and X. Wang, "Free vibration analysis of laminated composite and functionally graded sector plates with general boundary conditions," Composite Structures, vol. 132, pp. 720 736, 2015.

[52] F. Tornabene, E. Viola, and D. J. Inman, "2-D differential quadrature solution for vibration analysis of functionally graded conical, cylindrical shell and annular plate structures,"
Journal of Sound and Vibration, vol. 328, no. 3, pp. 259-290, 2009.

[53] F. Tornabene, E. Viola, and N. Fantuzzi, "General higher-order equivalent single layer theory for free vibrations of doublycurved laminated composite shells and panels," Composite Structures, vol. 104, pp. 94-117, 2013.

[54] F. Tornabene, N. Fantuzzi, M. Bacciocchi, and E. Viola, "A new approach for treating concentrated loads in doubly-curved composite deep shells with variable radii of curvature," Composite Structures, vol. 131, pp. 433-452, 2015.

[55] F. Tornabene, N. Fantuzzi, E. Viola, and J. N. Reddy, "WinklerPasternak foundation effect on the static and dynamic analyses of laminated doubly-curved and degenerate shells and panels," Composites Part B: Engineering, vol. 57, pp. 269-296, 2014.

[56] J. Du, W. L. Li, Z. Liu, T. Yang, and G. Jin, "Free vibration of two elastically coupled rectangular plates with uniform elastic boundary restraints," Journal of Sound and Vibration, vol. 330, no. 4, pp. 788-804, 2011. 


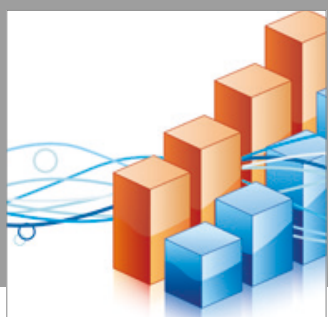

Advances in

Operations Research

vatersals

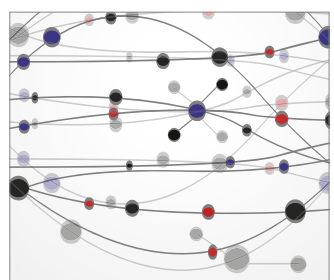

\section{The Scientific} World Journal
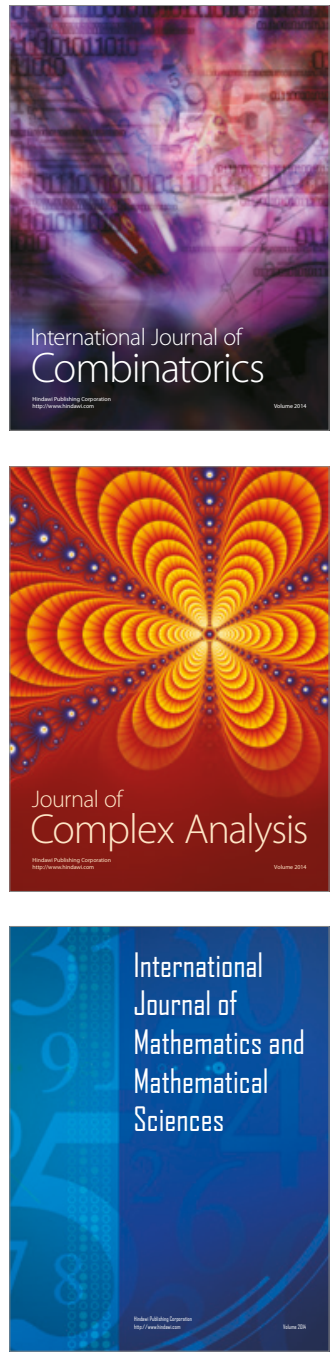
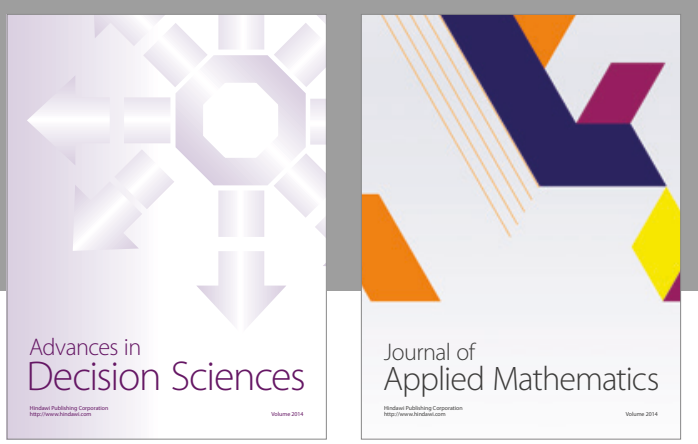

Algebra

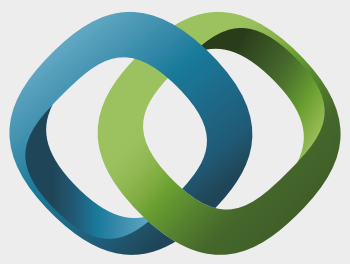

\section{Hindawi}

Submit your manuscripts at

https://www.hindawi.com
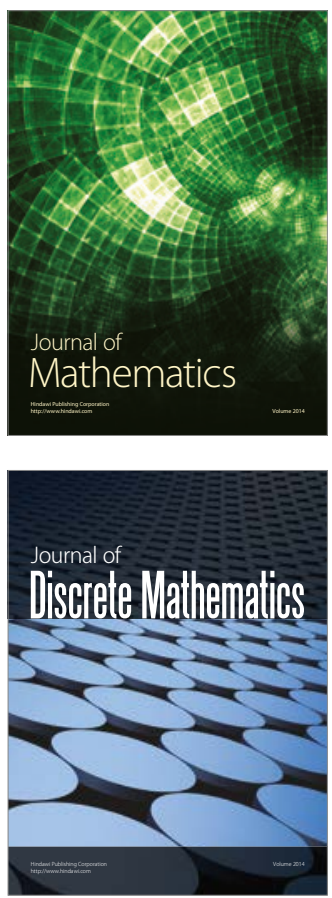

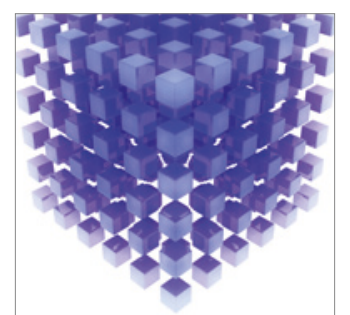

Mathematical Problems in Engineering
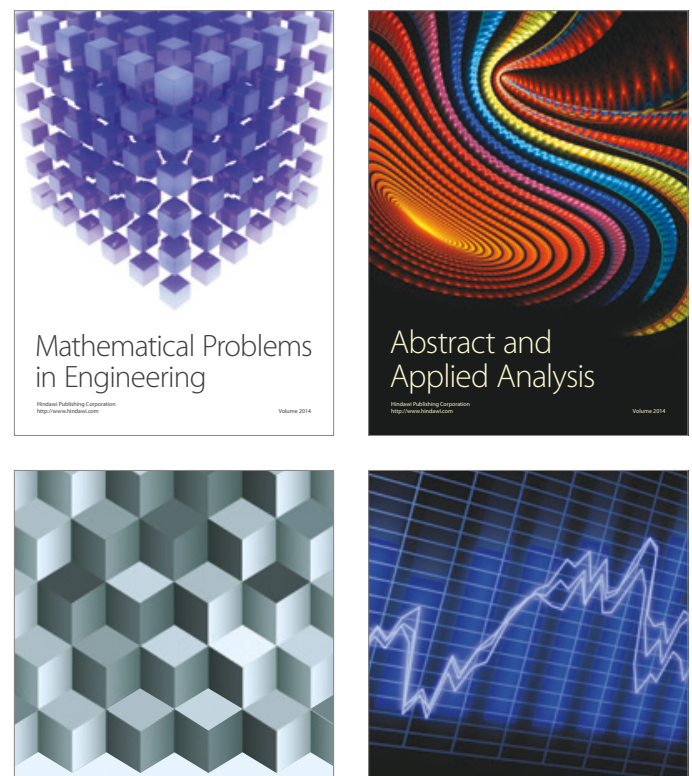

Journal of

Function Spaces

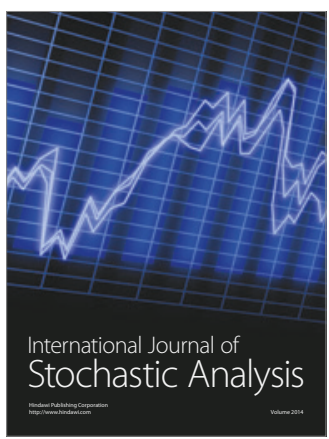

Probability and Statistics
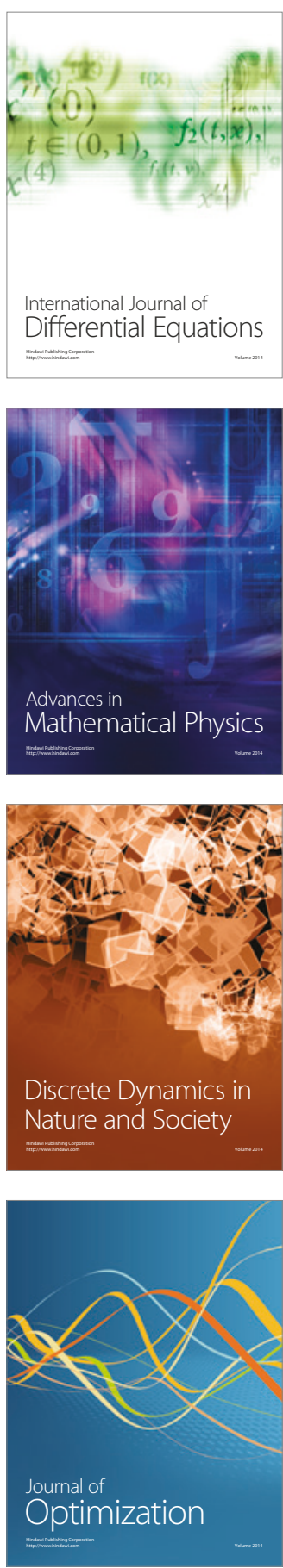